\title{
Dietary Patterns before and during Pregnancy and Gestational Age- and Sex-Specific Birth Weight: A Systematic Review
}

\author{
The Pregnancy and Birth to 24 Months Project \\ Published date: April, 152019
}

Nutrition Evidence Systematic Review

Center for Nutrition Policy and Promotion

Food and Nutrition Service

U.S. Department of Agriculture

3101 Park Center Drive

Alexandria, Virginia 
This systematic review was conducted for the Pregnancy and Birth to 24 Months Project (P/B-24 Project) by the Nutrition Evidence Systematic Review (NESR) team at the Center for Nutrition Policy and Promotion, Food and Nutrition Service, USDA. All systematic reviews from the P/B-24 Project are available on the NESR website: https://nesr.usda.gov.

Conclusion statements drawn as part of this systematic review describes the state of science related to the specific question examined. Conclusion statements do not draw implications, nor should they be interpreted to be dietary guidance.

The contents of this document may be used and reprinted without permission. Endorsement by NESR, the Center for Nutrition Policy and Promotion, the Food and Nutrition Service, or the U.S. Department of Agriculture of derivative products developed from this work may not be stated or implied.

In accordance with Federal civil rights law and U.S. Department of Agriculture (USDA) civil rights regulations and policies, the USDA, its Agencies, offices, and employees, and institutions participating in or administering USDA programs are prohibited from discriminating based on race, color, national origin, religion, sex, gender identity (including gender expression), sexual orientation, disability, age, marital status, family/parental status, income derived from a public assistance program, political beliefs, or reprisal or retaliation for prior civil rights activity, in any program or activity conducted or funded by USDA (not all bases apply to all programs). Remedies and complaint filing deadlines vary by program or incident.

Persons with disabilities who require alternative means of communication for program information (e.g., Braille, large print, audiotape, American Sign Language, etc.) should contact the responsible Agency or USDA's TARGET Center at (202) 720-2600 (voice and TTY) or contact USDA through the Federal Relay Service at (800) 877-8339. Additionally, program information may be made available in languages other than English.

To file a program discrimination complaint, complete the USDA Program Discrimination Complaint Form, AD3027, found online at How to File a Program Discrimination Complaint and at any USDA office or write a letter addressed to USDA and provide in the letter all of the information requested in the form. To request a copy of the complaint form, call (866) 632-9992. Submit your completed form or letter to USDA by: (1) mail: U.S. Department of Agriculture, Office of the Assistant Secretary for Civil Rights, 1400 Independence Avenue, SW, Washington, D.C. 20250-9410; (2) fax: (202) 690-7442; or (3) email: program.intake@usda.gov.

USDA is an equal opportunity provider, employer, and lender.

Suggested citation for this systematic review: Nutrition Evidence Systematic Review Team and Pregnancy Technical Expert Collaborative. Dietary Patterns before and during Pregnancy and Gestational Age- and Sex-Specific Birth Weight: A Systematic Review. Pregnancy and Birth to 24 Months Project. Alexandria, VA: U.S. Department of Agriculture, Food and Nutrition Service, Center for Nutrition Policy and Promotion, April 2019. Available at: https://nesr.usda.gov/project-specific-overview-pb-24-0

This systematic review has also been published in the American Journal of Clinical Nutrition: Raghavan R, Dreibelbis C, Kingshipp BL, Wong YP, Abrams B, Gernand AD, et al. Dietary patterns before and during pregnancy and birth outcomes: a systematic review. Am J Clin Nutr. 2019;109(7):729S-56S. doi: 10.1093/ajcn/nqy353.

\section{Related citations are published in the American Journal of Clinical Nutrition:}

- P/B-24 Project overview: Stoody EE, Spahn JM, Casavale KO. The Pregnancy and Birth to 24 Months Project: a series of systematic reviews on diet and health. Am J Clin Nutr. 2019;109(7):685S-97S. doi: 10.1093/ajcn/nqy372.

- P/B-24 systematic review methodology: Obbagy JE, Spahn JM, Wong YP, Psota TL, Spill MK, Dreibelbis C, et al. Systematic review methodology used in the Pregnancy and Birth to 24 Months Project. Am J Clin Nutr. 2019;109(7):698S-704S. doi: 10.1093/ajcn/nqy226

- Related systematic reviews from the P/B-24 Project: Raghavan R, Dreibelbis C, Kingshipp BL, Wong YP, Abrams B, Gernand AD, et al. Dietary patterns before and during pregnancy and maternal outcomes: a systematic review. Am J Clin Nutr. 2019;109(7):705S-28S. doi: 10.1093/ajcn/nqy216 


\section{Pregnancy Technical Expert Collaborative (TEC) members:}

- Barbara Abrams, DrPH, RD, University of California, Berkeley School of Public Health

- Anne Bartholomew, MS, RD, USDA

- Lisa M. Bodnar, PhD, MPH, RD, University of Pittsburgh School of Public Health

- Alison Gernand, PhD, MPH, RD The Pennsylvania State University

- Kathleen Rasmussen, ScD, RD, Cornell University

- Anna Maria Siega-Riz, PhD, University of Virginia School of Nursing

- Jamie S. Stang, PhD, MPH, RDN, University of Minnesota School of Public Health

\section{Nutrition Evidence Systematic Review (NESR) team:}

- Ramkripa Raghavani, DrPH, MPH, MSc, Panum Group, Lead analyst

- Carol Dreibelbisi, MPH, Panum Group, Analyst

- Brittany L. Kingshippi, PhD, Panum Group, Analyst (01/2017-Present)

- Yat Ping Wong, MLS, MPH, USDA, Librarian

\section{Project Leads:}

- Eve Essery Stoody, PhD, USDA

- Joanne M. Spahn, MS, RDN, USDA

- Kellie O. Casavale, PhD, RD, HHS

\section{Federal Expert Group (FEG)-Technical Expert Collaborative (TEC) Liaisons:}

- Anne Bartholomew, MS, RD, USDA

All TEC and NESR team members, Project leads, and FEG-TEC liaisons participated in establishing the research questions, analytic framework, and study inclusion and exclusion criteria. RR, CD, and YPW developed and conducted the literature search. $R R, C D$, and $B L K$ screened search results, identified studies for inclusion, extracted data, and assessed risk of bias for included studies. BA, AB, LMB, AG, KR, AMSR, JSS, EES, JMS, and KOC reviewed and provided substantive feedback on all systematic review materials, including the synthesis of the body of evidence, conclusion statement, and grade of the strength of the evidence. RR prepared this report and EES, JMS, and KOC provided oversight. All authors critically reviewed and approved the final report. The authors declare no conflicts of interest.

FUNDING SOURCE: United States Department of Agriculture, Food and Nutrition Service, Center for Nutrition Policy and Promotion, Alexandria, VA

i Under contract with the Food and Nutrition Service, United States Department of Agriculture. 


\section{TABLE OF CONTENTS}

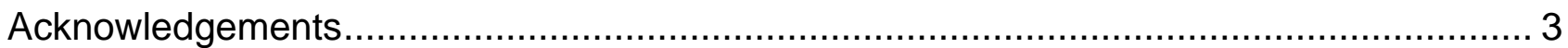

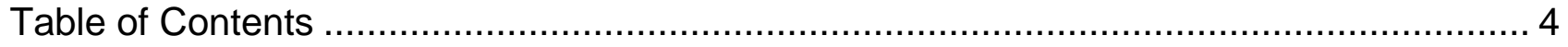

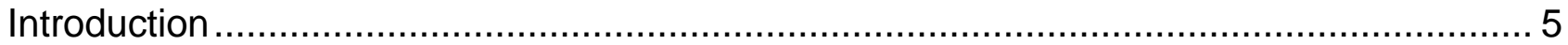

What is the relationship between dietary patterns before and during pregnancy and gestational age- and sex-specific birth weight? ......................................................... 7

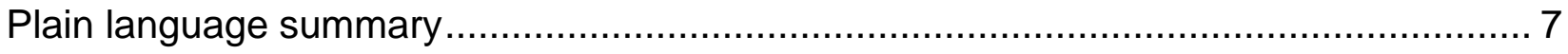

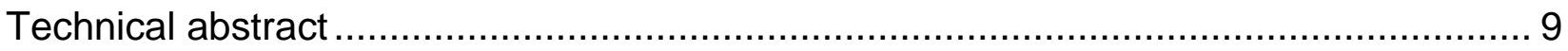

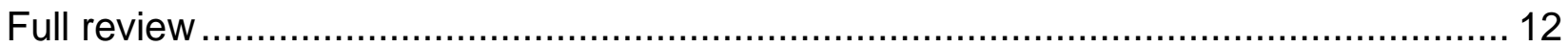

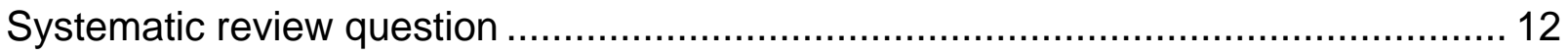

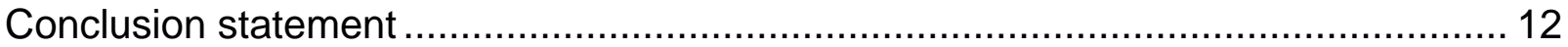

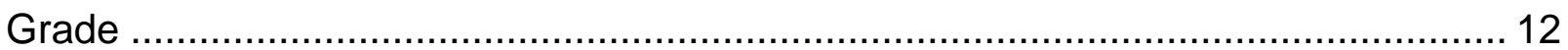

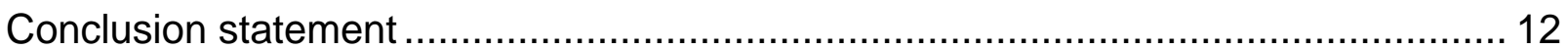

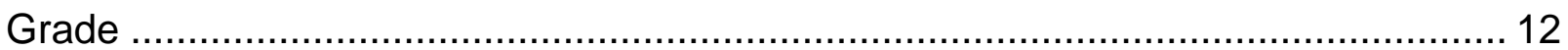

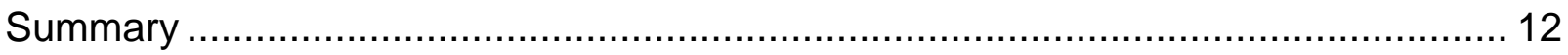

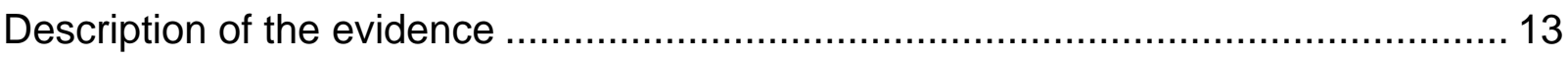

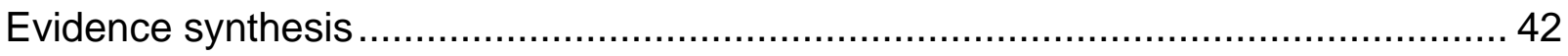

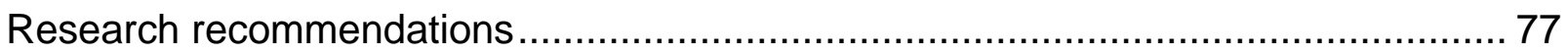

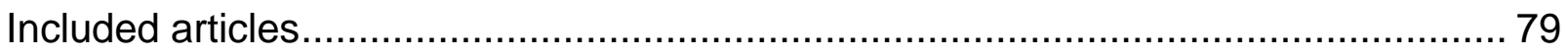

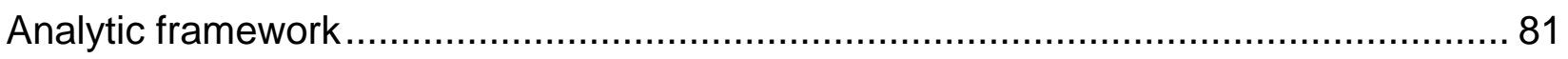

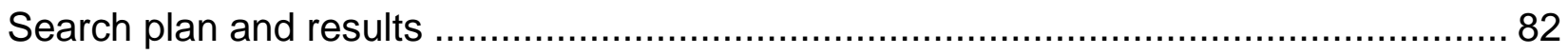

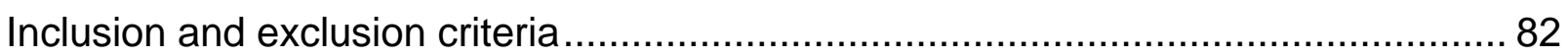

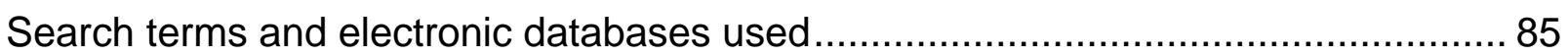

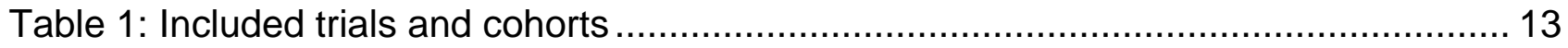

Table 2: Indices and scores used to assess the relationship between dietary patterns before and during pregnancy and gestational age- and sex-specific birth weight.............. 21

Table 3: Summary of dietary patterns identified using factor or principal components

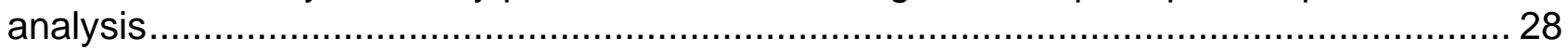

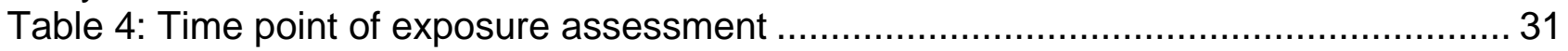

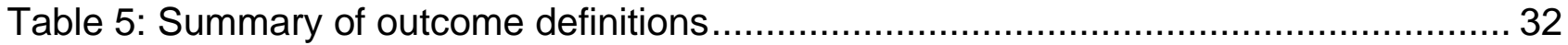

Table 6: Results grouped by methodology used for dietary pattern assessment............... 47

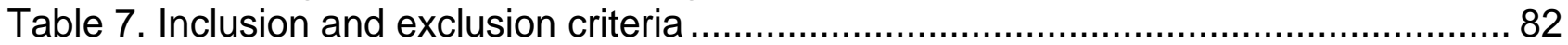

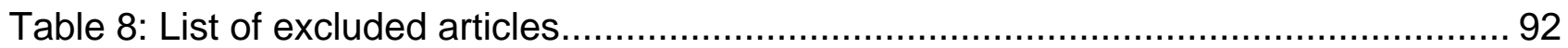

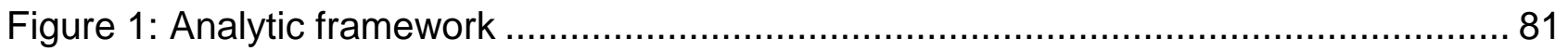

Figure 2: Flow chart of literature search and screening results.................................. 91 
This document describes a systematic review conducted to answer the following question: What is the relationship between dietary patterns before and during pregnancy and risk of hypertensive disorders of pregnancy? This systematic review was conducted as part of the Pregnancy and Birth to 24 Months (P/B-24) Project by USDA's Nutrition Evidence Systematic Review (NESR).

The purpose of the P/B-24 Project was to conduct a series of systematic reviews on diet and health for women who are pregnant and for infants and toddlers from birth to 24 months of age. This project was a joint initiative led by USDA and HHS, and USDA's NESR carried out all of the systematic reviews. A Federal Expert Group (FEG), a broadly representative group of Federal researchers and program leaders, also provided input throughout the P/B-24 Project. More information about the P/B-24 Project has been publishedii and is available on the NESR website: https://nesr.usda.gov/project-specificoverview-pb-24-0.

NESR, formerly known as the Nutrition Evidence Library (NEL), specializes in conducting food- and nutrition-related systematic reviews using a rigorous, protocol-driven methodology. To conduct each P/B-24 systematic review, NESR's staff worked with a Technical Expert Collaborative (TEC), which is a group of 7-8 leading subject matter experts.

NESR's systematic review methodology involves developing and prioritizing systematic review questions, searching for and selecting studies, extracting and assessing the risk of bias of data from each included study, synthesizing the evidence, developing a conclusion statement, grading the evidence underlying the conclusion statement, and recommending future research. A detailed description of the methodology used in conducting systematic reviews for the P/B-24 Project has been publishediii and is available on the NESR website: https://nesr.usda.gov/pb-24-project-methodology-0. In addition, starting on page 81, this document includes details about the methodology as it was applied to the systematic review described herein. An analytic framework that illustrates the overall scope of the question, including the population, the interventions and/or exposures, comparators, and outcomes of interest, is found on page 81. In addition, the literature search plan that was used to identify studies included in this systematic review is found on page 82.

\footnotetext{
ii Stoody EE, Spahn JM, Casavale KO. The Pregnancy and Birth to 24 Months Project: a series of systematic reviews on diet and health. Am J Clin Nutr. 2019;109(7):685S-97S. doi: 10.1093/ajen/nqy372.

iii Obbagy JE, Spahn JM, Wong YP, Psota TL, Spill MK, Dreibelbis C, et al. Systematic review methodology used in the Pregnancy and Birth to 24 Months Project. Am J Clin Nutr. 2019;109(7):698S704S. doi: 10.1093/ajcn/nqy226.
} 


\section{List of abbreviations}

\begin{tabular}{ll}
\hline Abbreviation & Full name \\
\hline FEG & Federal Expert Group \\
\hline HHS & Department of Health and Human Services \\
\hline NEL & Nutrition Evidence Library \\
\hline NESR & Nutrition Evidence Systematic Review \\
\hline P/B-24 & Pregnancy and Birth to 24 Months Project \\
\hline TEC & Technical Expert Collaborative \\
\hline USDA & United States Department of Agriculture \\
\hline
\end{tabular}




\section{WHAT IS THE RELATIONSHIP BETWEEN DIETARY PATTERNS BEFORE AND DURING PREGNANCY AND GESTATIONAL AGE- AND SEX- SPECIFIC BIRTH WEIGHT?}

\section{PLAIN LANGUAGE SUMMARY}

What is the question?

- The question is: What is the relationship between dietary patterns before and during pregnancy and gestational age- and sex-specific birth weight?

\section{What is the answer to the question?}

- No conclusion can be drawn on the association between dietary patterns during pregnancy and birth weight outcomes. Although research is available, the ability to draw a conclusion is restricted by

$\circ$ inconsistency in study findings,

- inadequate adjustment of birth weight for gestational age and sex, and

- variation in study design, dietary assessment methodology, and adjustment of key confounding factors.

- Insufficient evidence exists to estimate the association between dietary patterns before pregnancy and birth weight outcomes. There are not enough studies available to answer this question.

Why was this question asked?

- This important public health question was identified and prioritized as part of the U.S. Department of Agriculture and Department of Health and Human Services Pregnancy and Birth to 24 Months Project.

\section{How was this question answered?}

- A team of Nutrition Evidence Systematic Review staff conducted a systematic review in collaboration with a group of experts called a Technical Expert Collaborative.

What is the population of interest?

- Women who are pregnant or able to become pregnant, ages 15-44 years.

\section{What evidence was found?}

- This review includes 21 studies.

- These studies assessed the relationship between dietary patterns before and during pregnancy and birth weight outcomes.

- Only one-third of studies used both gestational age- and sex -specific cut-off values when defining birth weight outcomes.

- Findings were highly inconsistent across this body of evidence.

- Roughly half of studies found no association between dietary patterns and birth weight outcomes.

- Those that did find an association were inconsistent in the direction of effect and dietary patterns measured.

- There are limitations in the evidence as follows: inconsistencies across different 
studies in terms of study design, how dietary patterns were assessed and adjustment of key confounders.

- Additional research is needed to assess the relationship between dietary patterns before and during pregnancy and gestational age- and sex-specific birth weight.

\section{How up-to-date is this review?}

- This review includes literature from 01/1980 to 01/2017. 


\section{TECHNICAL ABSTRACT}

\section{Background}

- This systematic review was conducted as part of the U.S. Department of Agriculture and Department of Health and Human Services Pregnancy and Birth to 24 Months Project.

- The goal of this systematic review was to examine the following question: what is the relationship between dietary patterns before and during pregnancy and gestational age- and sex-specific birth weight.

\section{Conclusion Statement and Grades}

- No conclusion can be drawn on the association between dietary patterns during pregnancy and birth weight outcomes. Although research is available, the ability to draw a conclusion is restricted by

$\circ$ inconsistency in study findings,

○ inadequate adjustment of birth weight for gestational age and sex, and

- variation in study design, dietary assessment methodology, and adjustment of key confounding factors.

Grade: Grade not assignable

- Insufficient evidence exists to estimate the association between dietary patterns before pregnancy and birth weight outcomes. There are not enough studies available to answer this question.

Grade: Grade not assignable

\section{Methods}

- The systematic review was conducted by a team of staff from the Nutrition Evidence Systematic Review in collaboration with a Technical Expert Collaborative.

- Literature searches were conducted using PubMed, Embase, Cochrane, and other databases to identify studies that evaluated the relationship between dietary patterns before and during pregnancy and gestational age- and sexspecific birth weight. A manual search was conducted to identify articles that may not have been included in the electronic databases searched. Articles were screened by two authors independently for inclusion based on pre-determined criteria.

- Data from each included article were extracted, risks of bias were assessed, and both were checked for accuracy. The body of evidence was qualitatively synthesized, a conclusion statement was developed, and the strength of the evidence (grade) was assessed using pre-established criteria including evaluation of the internal validity/risk of bias, adequacy, consistency, impact, and generalizability of available evidence.

\section{Summary of Evidence}

- This systematic review includes18 prospective cohort, 1 retrospective cohort and 2 randomized control trials published between 1986 and 2016.

- The studies used multiple approaches to assess dietary patterns: 
- Nine studies used an index/score to assess dietary patterns.

- Eight studies used factor/principal component analysis (PCA).

- Two randomized controlled trials assigned subjects to one of two experimental diets.

- One study did not use a formal method to arrive at a dietary pattern.

- One study used both logistic regression and PCA.

- Many studies did not standardize for gestational age and/or infant sex when assessing birth weight.

- Just one-third of studies $(n=7)$ used both gestational age- and sex specific cut-off values when defining small for gestational age (SGA), large for gestational age (LGA), appropriate for gestational age (AGA), or intrauterine growth restriction (IUGR).

- Nine out of 21 studies reported birth weight, alone, without standardizing for gestational age or sex using z-scores.

- Study findings were highly inconsistent across the body of evidence. About half of studies $(n=10)$ found no association between dietary patterns and birth weight outcomes. Among studies that observed an association, there was limited consistency in direction of effect and the dietary patterns generated.

- There are serious limitations to the generalizability of this review. Minority, lower-SES, and adolescent populations are underrepresented in the body of evidence.

- The ability to draw strong conclusions was limited by the following issues:

- There was a lack of consistency in study findings.

- The data were primarily observational in nature, making it difficult to determine causal effect of the dietary patterns.

- Many studies did not adjust birth weight for gestational age and sex, and there was heterogeneity among the standardized measures that were used.

- The timing of exposure assessment and the duration of recall periods varied across studies.

- Key confounding factors were not consistently accounted for.

- None of the studies assessed effect measure modification between dietary patterns and maternal pre-pregnancy BMI in the context of birth weight outcomes.

- Many studies were conducted outside of the U.S.

- Adolescent, minority, and lower-SES populations were underrepresented.

- Additional research is needed that should:

- Include diverse populations from the U.S. and elsewhere with varying age groups (including adolescents) and different racial/ethnic and socioeconomic backgrounds.

- Assess effect measure modification by pre-pregnancy BMI and gestational weight gain.

- Use a standardized birth size measure (such as one developed by the INTERGROWTH- $21^{\text {st }}$ project) that would enable valid comparisons between and within countries ${ }^{1}$.

\footnotetext{
${ }^{1}$ Westerway, S. C., Papageorghiou, A. T., Hirst, J., Costa, F. D., Hyett, J., \& Walker, S. P. (2015).
} 
- Include well-designed and sufficiently powered RCTs.

- Foster collaborative efforts across different regions and populations so that dietary patterns can be more consistently scored, compared and reproduced across studies.

- Develop and validate novel epidemiological tools that can accurately capture the complexity of dietary habits.

- Promote harmonization of research methods across various cohorts and randomized trials, similar to the National Cancer Institute's Dietary Patterns Methods Project ${ }^{2}$.

- Adjust for key confounding factors in observational studies, including parity, educational attainment, smoking status, race/ethnicity, maternal age, family poverty income ratio, pre-pregnancy BMI, mean total energy intake and gestational weight gain. 


\section{FULL REVIEW}

\section{Systematic review question}

What is the relationship between dietary patterns before and during pregnancy and gestational age- and sex-specific birth weight?

\section{Conclusion statement}

No conclusion can be drawn on the association between dietary patterns during pregnancy and birth weight outcomes. Although research is available, the ability to draw a conclusion is restricted by

- inconsistency in study findings,

- inadequate adjustment of birth weight for gestational age and sex, and

- variation in study design, dietary assessment methodology, and adjustment of key confounding factors.

\section{Grade}

\section{Grade not assignable}

\section{Conclusion statement}

Insufficient evidence exists to estimate the association between dietary patterns before pregnancy and birth weight outcomes. There are not enough studies available to answer this question.

\section{Grade}

\section{Grade not assignable}

\section{Summary}

- This systematic review includes18 prospective cohort, 1 retrospective cohort and 2 randomized control trials published between 1986 and 2016.

- The studies used multiple approaches to assess dietary patterns:

- Nine studies used an index/score to assess dietary patterns.

- Eight studies used factor/principal component analysis (PCA).

- Two randomized controlled trials assigned subjects to one of two experimental diets.

- One study did not use a formal method to arrive at a dietary pattern.

- One study used both logistic regression and PCA.

- Many studies did not standardize for gestational age and/or infant sex when assessing birth weight.

$\circ$ Just one-third of studies $(n=7)$ used both gestational age- and sex specific cut-off values when defining small for gestational age (SGA), large for gestational age (LGA), appropriate for gestational age (AGA), or intrauterine growth restriction (IUGR).

- Nine out of 21 studies reported birth weight, alone, without standardizing for gestational age or sex using z-scores.

- Study findings were highly inconsistent across the body of evidence. About half of studies $(n=10)$ found no association between dietary patterns and birth weight outcomes. Among studies that observed an association, there was 
limited consistency in direction of effect and the dietary patterns generated.

- There are serious limitations to the generalizability of this review. Minority, lower-SES, and adolescent populations are underrepresented in the body of evidence.

\section{Description of the evidence}

- The search included articles from very high and high Human Development Index (HDI) countries, and the search timeframe spanned between January 1980 and January 2017.

- This evidence review includes 18 prospective cohort studies, 1 retrospective cohort study and 2 RCTs that examined the relationship between dietary patterns before and during pregnancy and gestational age- and sex-specific birth weight.

As described in

- Table 1: Included trials and cohorts, a variety of cohorts and trials were represented in this body of evidence.

Table 1: Included trials and cohorts

Cohort/Trial Name

Study

\section{Cardiovascular Risk Reduction Diet in Pregnancy (CARRDIP) trial}

2. Generation $\mathbf{R}$

$(2,3)$

3. Australian Longitudinal Study on Women's Health

4. Norwegian Mother and Child Cohort (MoBA)

5. Infant Feeding Practices Study (IFPS II)

6. INMA (Infancia y medio ambiente) and Rhea study

7. Valencia birth cohort (a sub-project of the INMA cohort)

8. Project Viva

9. Healthy Start Study

10. Growing up in Singapore Towards Healthy Outcomes Study (GUSTO)

11. CANDLE Study (Conditions Affecting Neurocognitive Development and Learning in Early Childhood)

12. Danish National Birth Cohort 
13. Born in Guangzhou Cohort Study

14. Avon Longitudinal Study of Parents and Children

15. Osaka Maternal and Child Health Study

16. National Longitudinal Study of Adolescent Health (Add Health)

17. Unnamed cohort

$(18-20)$

\section{Unnamed trial}

- Seven of the 21 studies were conducted in the U.S $(6,9,10,12,17,18,21)$. In addition, two studies were conducted in Spain $(8,19)$, one in Spain and Greece (7), two in Netherlands $(2,3)$, two in Australia $(4,20)$, two in Norway $(1,5)$, one in Denmark (13), one in Singapore (11), one in China (14), one in Japan (16) and one in England (15). See the map below.

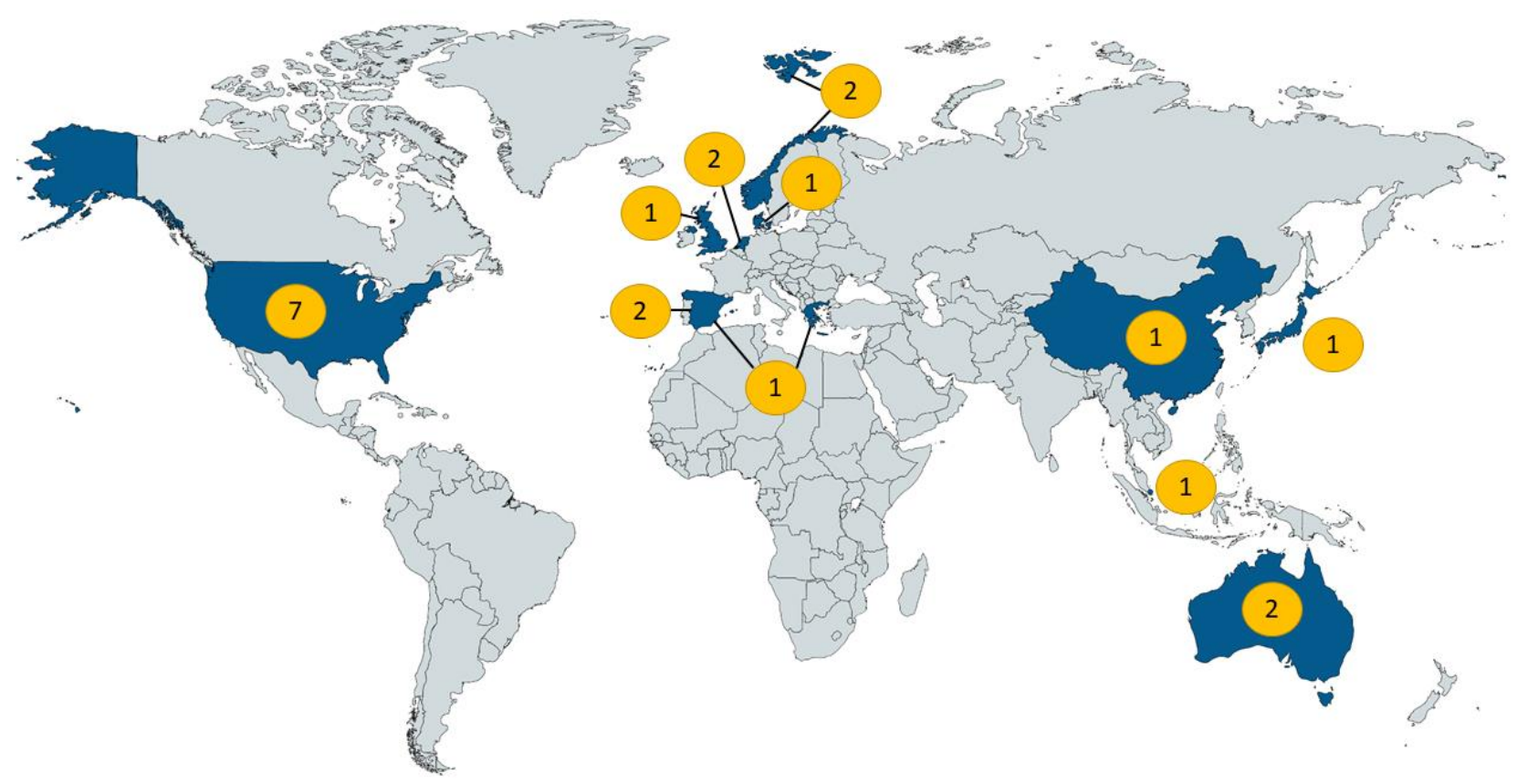

Subject characteristics:

- Sample size of the studies ranged from 12 subjects in a pilot study (21) to 66,597 subjects (5). The median sample size was 1,079 .

- Age: Most of the studies included women between 20 and 40 years of age. An exception was the Add Health cohort, which included only adolescent participants (17). Monteagudo et al. reported that their study participants were between ages 21-46 years and the mean age was 31.9 years. Other studies noted that anywhere between $10 \%$ and $29 \%$ of the study participants were at 
least 35 years of age. The percentage of participants over 40 years was not specified, except by Knudsen et al. (1\% of participants). The rest of the studies presented the mean age, which ranged from $\sim 27(12)$ to $\sim 35$ (21).

- Pregnancy characteristics: Almost all of the studies included singleton pregnancies, only.

- Health Characteristics:

- One-third of studies excluded subjects with a previous diagnosis of type 1 and/or 2 diabetes mellitus (1, 5, 6, 10, 11, 14, 19). In addition, Shapiro et al. also excluded subjects with gestational diabetes in their index pregnancy.

- A few studies included mother-baby dyads only if the baby was born within a specific time period:

- Hillesund et al. included babies born between 37 and 42 weeks.

- Poon et al. included children born at or after 35 weeks of gestation, but before 43.5 weeks. In addition, Poon et al. specified that only babies who weighed $\geq 5 \mathrm{lbs}$ and didn't stay in the intensive care unit for more than 3 days were included.

- Shapiro et al. included all children with a gestational age $\geq 32$ weeks.

- Knudsen et al. included only full-term infants.

- Lu et al. excluded preterm deliveries.

- Okubo et al. included term deliveries born between 37 and 41 weeks of gestation.

- A few studies excluded subjects with the following chronic diseases:

- Hypertension $(1,8,14,19)$

- Thyroid pathology (19)

- Asthma with active steroid management (10)

- Timmermans et al. reported that $6 \%$ of their subjects had co-morbidities including chronic hypertension, heart disease, diabetes, high cholesterol, thyroid disease and systemic lupus erythematosus. Similarly, Chatzi noted that $<3 \%$ of their study participants had gestational hypertension and $<1 \%$ had diabetes before pregnancy.

- Khoury et al. excluded women with high risk pregnancies caused by endocrine disease, history of thromboembolic disease or significant gastrointestinal, cardiac, pulmonary, or hematologic disease. Women with complications during a previous pregnancy, including neonatal death, still birth, and preterm delivery, were also excluded. Women who experienced ongoing hyperemesis gravidarum or bleeding after gestational week 12 in the current pregnancy were not included.

- Okubo et al. reported that $\sim 13 \%$ of their subjects had one or more of the following conditions: Hyperemesis, hydramnios, oligoamnios, gestosis, abruptio placenta, placenta previa, incompetent cervical os or others diagnosed by a medical doctor.

- A few studies also excluded subjects with drug-abuse $(1,3)$ or serious psychiatric illness (10), or who were receiving psychotropic drugs (11).

- Four studies excluded subjects whose pregnancies were conceived using assisted reproductive technologies $(2,3,7,8)$.

- Two studies noted that their study participants were healthy or had a low risk pregnancy $(12,21)$. 
- Smoking during pregnancy varied across studies:

$\circ$ Daily smoking ranged between $\sim 3 \%$ (11) and 32\% (20). Lu et al. reported that $30 \%$ experienced passive smoking.

- Khoury et al. excluded current smokers from their study.

- Pre-pregnancy smoking status (7-13 years prior to becoming pregnant) was reported to be $25 \%$ in one study (4).

- Race/ethnicity: About half of the studies $(n=10)$ did not report participants' race/ethnicity. Among the studies that did report this information:

- One-third of studies noted that a majority of their subjects were white (Khoury: 100\%; Grieger: 89\%; Rodriguez-Bernal: 88\%; Poon: 87\%; Northstone: 82\%; Rifas-Shiman: 72\%; Shapiro: 55\%). Categorized another way, Xie et al. noted that $77 \%$ of the study subjects were nonBlacks. Bouwland-Both et al. conducted their study in an all-Dutch population.

- Only two studies reported including a majority of non-white subjects:

- Colon-Ramos noted that $62 \%$ of their study participants were African American.

- The GUSTO study participants (based in Singapore) were Chinese, Malayan or Indian. The authors did not specify the overall percentage of each race/ethnicity.

- Parity: There was heterogeneity in terms of study participants' parity. For example, in some studies, most subjects were nulliparous (88\% in Lu et al., $90 \%$ in Gresham et al. and $100 \%$ in Xie et al.). Other studies $(n=11)$ reported that $\sim 38 \%$ to $68 \%$ of women were nulliparous.

- Pre-pregnancy BMI:

- In this body of evidence, Chia et al. reported one of the lowest mean prepregnancy BMls (which ranged between 22.1 and $22.7 \mathrm{~kg} / \mathrm{m}^{2}$, depending on the dietary pattern quintile). While Okubo et al. noted that the mean pre-pregnancy BMI was $20.2 \mathrm{~kg} / \mathrm{m}^{2}$, it was based on maternal body weight measured at 20 years of age.

- Colon-Ramos reported one of the highest mean pre-pregnancy BMI $\left(\sim 27.6 \mathrm{~kg} / \mathrm{m}^{2}\right)$, with the African American sub-group noting even higher mean BMl $\left(28.8 \mathrm{~kg} / \mathrm{m}^{2}\right)$. The percentage of participants with BMI over 30 $\mathrm{kg} / \mathrm{m}^{2}$ ranged from $4 \%(15)$ to $19 \%(10)$.

- It should be noted that Khoury et al. excluded subjects with a BMl either $<19$ or $>32 \mathrm{~kg} / \mathrm{m}^{2}$.

- While Grieger et al. reported on maternal BMI, it is unclear if it was measured pre-pregnancy.

- Xie et al. reported that pre-pregnancy BMI in adolescents was 22.2 $\mathrm{kg} / \mathrm{m}^{2}$.

- Maternal education: Among studies that reported maternal education $(n=16)$, participants with more than a high school education ranged from $16 \%$ (19) to 91\% (9).

- Socioeconomic status (SES) was reported in some of the studies $(n=14)$. Data presented across studies were heterogeneous. Below are a few examples of studies that reported data on SES.

- Kennedy noted that all of their participants were WIC eligible (income $<185 \%$ of poverty level).

- Poon noted that $38 \%$ had income $<185 \%$ of poverty level. 
- Rifas-Shiman reported that $13 \%$ of their participants had income below $\$ 40,000$, whereas Shapiro et al. reported that $29 \%$ of participants had income below $\$ 40,000$.

- Rodriguez-Bernal noted that $61 \%$ of their participants were manual workers. Meanwhile, Monteagudo et al. reported that $37 \%$ of participants were employed doing housework and $5 \%$ did agricultural work.

- Grieger et al. reported that $82 \%$ of their participants were in the lowest $2 / 5^{\text {th }}$ SES strata.

- Xie et al. study participants were adolescents, so they reported parental education status as a proxy for SES, with $79 \%$ of parents having at least a high school education.

- Although Colon-Ramos did not report the SES of the study participants, the authors mentioned that the study was conducted in a U.S. county that was primarily low income (<200\% poverty level).

\section{Interventions/Exposures:}

Dietary patterns were assessed using 1) index/score analysis; 2) factor analysis and PCA; 3) experimental diet; 4) PCA and reduced rank regression; and 5) another unspecified method. A description of the studies included by each method used to measure dietary patterns is included below.

- Index/score analysis (Table 2: Indices and scores used to assess the relationship between dietary patterns before and during pregnancy and birth weight): Nine studies included in this review used one or more of the following indices/scores:

- Mediterranean Diet Scale (7)

- Australian Recommended Food Score (4)

- New Nordic Diet score (5)

- Nutritional Risk Score (18)

- Mediterranean Diet Score for Pregnancy (19)

- Alternative Mediterranean Diet (aMED) (6)

- Alternative Healthy Eating Index for Pregnancy $(6,8,9)$

- Healthy Eating Index-2010 (10)

- Factor analysis and PCA (Table 3: Summary of dietary patterns identified using factor or principal components analysis): Eight studies included in this review assessed dietary patterns using factor analysis or PCA $(3,11-16$, 20)

- Experimental diet: Two studies included in this review assigned participants to one of two experimental diets $(1,21)$. Khoury et al. randomly assigned participants to the following diets:

o Intervention diet:

- Dietitians encouraged the intake of fatty fish, vegetable oils, especially olive oil and rapeseed oil, nuts, nut butters, margarine based on olive- or rapeseed oil, and avocado to replace meat, butter, cream, and fatty dairy products; the consumption of fresh fruits and vegetables was advised (at least 6 a day); intake of dairy products in the form of skimmed or low-fat products (skimmed milk, fat-reduced cheese, and yogurt) in place of full fat products was encouraged; subjects were advised to choose meat for a main meal twice a week and use legumes, vegetable main 
dishes, fatty fish, or poultry with the fat trimmed off on the other days; coffee was limited to 2 cups of filtered coffee a day

- Compliance data showed that the intervention group included significantly more fish and fish products; fatty fish and fish products; rapeseed-based margarine; oils; olive oil; rapeseed oil; nuts, olives, and seeds; vegetables; and fruits when compared to the control diet ${ }^{3}$

- Control diet:

- Subjects were asked to consume their usual diet based on Norwegian foodstuffs, and not to introduce more oils or low-fat meat and dairy products than usual

- Included significantly more fatty milk, meat and meat products, fatty minced meat, butter, and hard margarines when compared to the intervention $\operatorname{diet}^{6}$

In a pilot RCT, Clapp randomly assigned participants to one of two experimental diets:

- Aboriginal carbohydrate diet (Low-Gl): used carbohydrates from unprocessed whole grains, fruits, beans, vegetables, and many dairy products; includes most dense whole grain and multigrain breads, bran cereals, pastas, fresh fruits and vegetables, yogurt, ice cream, and nuts

- Cafeteria carbohydrate diet (High-Gl): used carbohydrates from highly processed grains, root vegetables, and simple sugars; includes many highly-refined breads, potatoes, instant rice, most breakfast cereals, desserts, and snack-type foods.

- Logistic Regression and PCA: One study assessed adherence to a Mediterranean diet using logistic regression and PCA (2).

- Unspecified method: One study created dietary patterns by grouping foods into categories defined "on the basis of theoretical expectations" by Dube et al. ${ }^{4}$ and later used by Jeffery et al. ${ }^{5}$ and Agurs-Collins et al ${ }^{6}$ (17). The following three categories were created:

- High-calorie sweet pattern: includes foods such as doughnuts, ice cream, chocolate candy, regular candy, and cookies

- High-calorie nonsweet pattern: includes steak, fried chicken, fried fish, pizza, hot dogs, sausage, cheese, whole milk, etc.

- Low-calorie pattern: includes foods such as low-fat and skim milk, grilled chicken, grilled fish, apples, and breakfast cereal

\section{Time point of exposure:}

As described below, the time point of exposure and recall periods were heterogeneous across studies (Table 4: Time point of exposure assessment). The following section

\footnotetext{
${ }^{3}$ Khoury, J., Henriksen, T., Seljeflot, I., Mørkrid, L., Frøslie, K. F., \& Tonstad, S. (2007). Effects of an antiatherogenic diet during pregnancy on markers of maternal and fetal endothelial activation and inflammation: the CARRDIP study. BJOG, 114(3), 279-288. doi:10.1111/j.1471-0528.2006.01187.x

4 Dube, L., LeBel, J. L., \& Lu, J. (2005). Affect asymmetry and comfort food consumption. Physiol Behav, 86(4), 559-567. doi:10.1016/j.physbeh.2005.08.023

5 Jeffery, R. W., Linde, J. A., Simon, G. E., Ludman, E. J., Rohde, P., Ichikawa, L. E., \& Finch, E. A. (2009). Reported food choices in older women in relation to body mass index and depressive symptoms. Appetite, 52(1), 238-240. doi:10.1016/j.appet.2008.08.008

${ }^{6}$ Agurs-Collins, T., \& Fuemmeler, B. F. (2011). Dopamine polymorphisms and depressive symptoms predict foods intake. Results from a nationally representative sample. Appetite, 57(2), 339-348. doi:10.1016/j.appet.2011.05.325
} 
discusses the time period diet was assessed and the time window it represents.

- Before pregnancy:

- Grieger et al. (20) collected data at 13 weeks, but the recall period was 12 months prior to conception.

- Monteagudo et al. (19), Gresham et al. (4) and Xie et al. (17) did not specify a particular time period when the diet was assessed (which could have ranged from before to during pregnancy).

- First trimester: The following studies assessed diet during the first trimester, only:

- Rodriguez-Bernal et al. (8)

- Bouwland-Both et al. (3)

- Timmermans et al. (2)

- Second trimester: The following studies assessed diet during the second trimester, only:

- Hillesund et al. (5)

- Chia et al. (11)

- Colon-Ramos et al. (12)

- Knudsen et al. (13)

- Lu et al. (14)

- Third trimester: Northstone et al. (15) assessed diet during the third trimester, only.

- In the following studies, diet measurement spanned multiple trimesters -

- Rifas-Shiman et al. (9) assessed maternal diet at two distinct time points: first trimester (11.7 \pm 3.1 weeks) and second trimester (26 to 28 weeks)

- Poon et al. (6) assessed diet in a window of 26 to 36 weeks.

- Chatzi et al. (7) used multiple cohorts, with INMA collecting maternal diet data during the first trimester $(\sim 13.8 \pm 2$ weeks) and Rhea collecting dietary data between 14 and 18 weeks.

- Shapiro et al. (10) collected dietary data one to eight times between 8 and 24 weeks.

- Although Okubo et al. (16) collected data at around 18 weeks, the range spanned between 5 and 39 weeks.

- Clapp (21) assigned diets randomly around 8 weeks and followed them until delivery.

- Khoury et al. (1) assigned participants to one of two experimental diets between 17 and 20 weeks and followed them until delivery.

- The recall period varied from a few days $(10,11,17)$ to a few weeks $(13,14)$ to a few months $(2,3,5-9,12,16)$.

\section{Outcomes:}

Studies assessed several outcomes including birth weight (reported in grams or kilograms), low birth weight, weight-for-length and weight-for-age z scores, macrosomia, SGA, AGA, LGA, body composition, and IUGR. Some studies also assessed fetal growth measures, including crown-rump length, estimated fetal weight, fetal head circumference, abdominal circumference, femur length, and placental resistance measured using pulsatility index umbilical artery and resistance index uterine artery. Many studies did not standardize for gestational age or sex when assessing birth weight (i.e., they used "raw" birth weight as the outcome across a 
range of preterm and term births). Specifically, one-third of studies $(n=7)$ used both gestational age and sex-specific cut-off values when defining SGA, AGA, LGA and IUGR $(1,5,6,11,13,14,16)$. Table 5: Summary of outcome definitions, summarizes the outcomes and diagnostic criteria grouped by methodology used to create dietary patterns. 
Table 2: Indices and scores used to assess the relationship between dietary patterns before and during pregnancy and birth weight

\begin{tabular}{|c|c|c|c|c|}
\hline $\begin{array}{l}\text { Index/Score } \\
\text { (Reference) }\end{array}$ & $\begin{array}{l}\text { Mediterranean diet }{ }^{10} \\
\text { (min-max score) }\end{array}$ & $\begin{array}{l}\text { Australian } \\
\text { Recommended Food } \\
\text { Score } \\
\text { (min-max score) }\end{array}$ & $\begin{array}{l}\text { New Nordic Diet } \\
\text { Score }{ }^{12,13}\end{array}$ & $\begin{array}{l}\text { Nutritional Risk } \\
\text { Score }{ }^{14,15} \\
\text { (min-max score) }\end{array}$ \\
\hline Article & Chatzi et al., 2012 & Gresham et al., 2016 & Hillesund et al., 2014 & Kennedy, 1986 \\
\hline Component & Total score: 0-8 & Total score: $0-72$ & Total score: $0-10$ & Total score: 0-100 \\
\hline \multirow[t]{3}{*}{ Vegetables } & \multirow[t]{3}{*}{$\begin{array}{l}\text { Vegetables } \\
\geq \text { Median }=1 ;<\text { Median }=0\end{array}$} & \multirow[t]{3}{*}{$\begin{array}{l}\text { Vegetables } \\
(0-22)\end{array}$} & $\begin{array}{l}\text { Root Vegetables } \\
\geq \text { Median }=1 ;<\text { Median }=0\end{array}$ & \multirow{3}{*}{$\begin{array}{l}\text { Fruits and vegetables } \\
(0-30)\end{array}$} \\
\hline & & & $\begin{array}{l}\text { Cabbages } \\
\geq \text { Median }=1 ;<\text { Median }=0\end{array}$ & \\
\hline & & & $\begin{array}{l}\text { Potatoes relative to rice } \\
\text { and pasta combined } \\
\geq \text { Median }=1 ;<\text { Median }=0\end{array}$ & \\
\hline \multirow[t]{2}{*}{ Fruits } & \multirow[t]{2}{*}{$\begin{array}{l}\text { Fruits and nuts } \\
\geq \text { Median }=1 ;<\text { Median }=0\end{array}$} & \multirow[t]{2}{*}{$\begin{array}{l}\text { Fruits } \\
(0-14)\end{array}$} & $\begin{array}{l}\text { Nordic fruits } \\
\geq \text { Median }=1 ;<\text { Median }=0\end{array}$ & \multirow[t]{2}{*}{$\begin{array}{l}\text { Fruits included with } \\
\text { vegetables above }\end{array}$} \\
\hline & & & $\begin{array}{l}\text { Native berries (includes } \\
\text { "foods from wild } \\
\text { countryside") }\end{array}$ & \\
\hline
\end{tabular}

10 Trichopoulou A, Costacou T, Bamia C, et al. (2003) Adherence to a Mediterranean diet and survival in a Greek population. N Engl J Med 348, $2599-2608$.

${ }^{11}$ Collins CE, Young AF \& Hodge A (2008) Diet quality is associated with higher nutrient intake and self-rated health in mid-aged women. J Am Coll Nutr $27,146-157$.

12 Includes meal frequency, which is not included as a component in this table.

${ }^{13}$ Mithril C, Dragsted LO, Meyer C et al. (2012) Guidelines for the New Nordic Diet. Public Health Nutr 15, 1941-1947.

14 No reference provided.

15 Supplements and 'other foods and beverages' were also measured but not included in the score 


\begin{tabular}{|c|c|c|c|c|}
\hline $\begin{array}{l}\text { Index/Score } \\
\text { (Reference) }\end{array}$ & $\begin{array}{l}\text { Mediterranean diet }{ }^{10} \\
\text { (min-max score) }\end{array}$ & $\begin{array}{l}\text { Australian } \\
\text { Recommended Food } \\
\text { Score }{ }^{11} \\
\text { (min-max score) }\end{array}$ & $\begin{array}{l}\text { New Nordic Diet } \\
\text { Score }{ }^{12,13}\end{array}$ & $\begin{array}{l}\text { Nutritional Risk } \\
\text { Score }{ }^{14,15} \\
\text { (min-max score) }\end{array}$ \\
\hline \multirow{2}{*}{$\begin{array}{l}\text { Cereals/ } \\
\text { Grains and } \\
\text { whole grains }\end{array}$} & $\begin{array}{l}\text { Cereals } \\
\geq \text { Median }=1 ;<\text { Median }=0\end{array}$ & $\begin{array}{l}\text { Grains } \\
(0-14)\end{array}$ & $\begin{array}{l}\text { Whole grain bread } \\
\geq \text { Median }=1 ;<\text { Median }=0\end{array}$ & $\begin{array}{l}\text { Bread and cereal } \\
(0-15)\end{array}$ \\
\hline & & & $\begin{array}{l}\text { Oatmeal porridge } \\
\geq \text { Median }=1 ;<\text { Median }=0\end{array}$ & \\
\hline $\begin{array}{l}\text { Nuts and } \\
\text { legumes }\end{array}$ & $\begin{array}{l}\text { Legumes } \\
\geq \text { Median }=1 ;<\text { Median }=0 \\
\text { Nuts included } \text { with fruits }\end{array}$ & $\begin{array}{l}\text { See under fish and other } \\
\text { protein foods }\end{array}$ & & $\begin{array}{l}\text { Nuts, peanut butter, and } \\
\text { legumes included under } \\
\text { meat }\end{array}$ \\
\hline Meat & $\begin{array}{l}\text { Meat } \\
\geq \text { Median = 0; }<\text { Median = } 1 \\
\text { (reverse scored) }\end{array}$ & $\begin{array}{l}\text { Meat included as part of } \\
\text { protein }\end{array}$ & & $\begin{array}{l}\text { Meat or alternatives } \\
(0-40)\end{array}$ \\
\hline \multirow[t]{3}{*}{$\begin{array}{l}\text { Fish and other } \\
\text { protein foods }\end{array}$} & $\begin{array}{l}\text { Fish and seafood } \\
\geq \text { Median }=1 ;<\text { Median }=0\end{array}$ & $\begin{array}{l}\text { Fish } \\
(0-2)\end{array}$ & $\begin{array}{l}\text { Foods from wild } \\
\text { countryside (wild fish, } \\
\text { seafood, game) }{ }^{16} \\
\text { Also includes native } \\
\text { berries, captured as part } \\
\text { of fruits } \\
\geq \text { Median }=1 ; \text {; } \text { Median }=0\end{array}$ & $\begin{array}{l}\text { Fish, liver, and eggs } \\
\text { included as part of meat }\end{array}$ \\
\hline & & $\begin{array}{l}\text { Meat } \\
(0-5)\end{array}$ & & \\
\hline & & $\begin{array}{l}\text { Nuts/bean/soya } \\
(0-6)\end{array}$ & & \\
\hline
\end{tabular}

${ }^{16}$ Salmon and Trout excluded 


\begin{tabular}{|c|c|c|c|c|}
\hline $\begin{array}{l}\text { Index/Score } \\
\text { (Reference) }\end{array}$ & $\begin{array}{l}\text { Mediterranean diet }{ }^{10} \\
\text { (min-max score) }\end{array}$ & $\begin{array}{l}\text { Australian } \\
\text { Recommended Food } \\
\text { Score }{ }^{11} \\
\text { (min-max score) }\end{array}$ & $\begin{array}{l}\text { New Nordic Diet } \\
\text { Score }^{12,13}\end{array}$ & $\begin{array}{l}\text { Nutritional Risk } \\
\text { Score }{ }^{14,15} \\
\text { (min-max score) }\end{array}$ \\
\hline & & $\begin{array}{l}\text { Eggs } \\
(0-1)\end{array}$ & & \\
\hline Dairy & $\begin{array}{l}\text { Dairy products } \\
\geq \text { Median }=1 ;<\text { Median }=0\end{array}$ & $\begin{array}{l}\text { Dairy } \\
(0-7)\end{array}$ & $\begin{array}{l}\text { Milk } \\
\geq \text { Median = } 1 ;<\text { Median }=0\end{array}$ & $\begin{array}{l}\text { Milk and cheese } \\
(0-15)\end{array}$ \\
\hline Fat & $\begin{array}{l}\text { MUFA:SFA ratio } \\
\geq \text { Median }=1 ;<\text { Median }=0\end{array}$ & $\begin{array}{l}\text { Fat } \\
(0-1)\end{array}$ & & \\
\hline \multicolumn{5}{|l|}{$\begin{array}{l}\text { Empty } \\
\text { calories }\end{array}$} \\
\hline \multicolumn{5}{|l|}{ Fiber } \\
\hline \multicolumn{5}{|c|}{ Micronutrients } \\
\hline \multicolumn{5}{|l|}{$\begin{array}{l}\text { Sweetened } \\
\text { beverages }\end{array}$} \\
\hline Water & & & $\begin{array}{l}\text { Water consumption } \\
\text { relative to sweetened } \\
\text { beverages } \\
\geq \text { Median }=1 ;<\text { Median }=0\end{array}$ & \\
\hline Alcohol & Excluded alcohol & Excluded alcohol & & \\
\hline
\end{tabular}




\begin{tabular}{|c|c|c|c|c|c|}
\hline $\begin{array}{l}\text { Index/Score } \\
\text { (Reference) }\end{array}$ & $\begin{array}{l}\text { Mediterranean } \\
\text { Diet Score for } \\
\text { Pregnancy }{ }^{17}\end{array}$ & $\begin{array}{l}\text { AHEI-P } \\
\text { (min-max } \\
\text { score) }\end{array}$ & $\begin{array}{l}\text { Alternate } \\
\text { Mediterranean } \\
\text { Diet }^{19}\end{array}$ & $\begin{array}{l}\text { AHEI-P } \\
\text { (min-max } \\
\text { score) }\end{array}$ & HEI-2010 21 \\
\hline Article & $\begin{array}{l}\text { Monteagudo et } \\
\text { al., } 2016\end{array}$ & Poon et al., 2013 & Poon et al., 2013 & $\begin{array}{l}\text { Rifas-Shiman et } \\
\text { al., 2009; } \\
\text { Rodriguez-Bernal } \\
\text { et al., } 2010\end{array}$ & $\begin{array}{l}\text { Shapiro et al., } \\
2016\end{array}$ \\
\hline Component & Total score: 4-11 & Total score: 0-130 & Total score: 0-8 & Total score: 0-90 & Total score: $0-100$ \\
\hline \multirow[t]{2}{*}{ Vegetables } & $\begin{array}{l}\text { Vegetables } \\
\geq \text { Median }=1 \\
<\text { Median }=0\end{array}$ & $\begin{array}{l}\text { Vegetables } \\
(0-10)\end{array}$ & $\begin{array}{l}\text { Vegetables } \\
\geq \text { Median }=1 \\
<\text { Median }=0\end{array}$ & $\begin{array}{l}\text { Vegetables } \\
(0-10) \\
\text { (Includes tofu and } \\
\text { soybeans) }\end{array}$ & $\begin{array}{l}\text { Total vegetables } \\
(0-5)\end{array}$ \\
\hline & & & & & $\begin{array}{l}\text { Greens and } \\
\text { beans } \\
(0-5)\end{array}$ \\
\hline \multirow[t]{2}{*}{ Fruits } & $\begin{array}{l}\text { Fruits and nuts } \\
\geq \text { Median = } 1 ; \\
<\text { Median }=0\end{array}$ & $\begin{array}{l}\text { Whole fruit } \\
(0-10)\end{array}$ & $\begin{array}{l}\text { Fruits } \\
\geq \text { Median }=1 \\
<\text { Median }=0\end{array}$ & $\begin{array}{l}\text { Fruits } \\
(0-10)\end{array}$ & $\begin{array}{l}\text { Total fruit } \\
(0-5)\end{array}$ \\
\hline & & & & & $\begin{array}{l}\text { Whole fruit } \\
(0-5)\end{array}$ \\
\hline $\begin{array}{l}\text { Cereals/ Grains } \\
\text { and whole grains }\end{array}$ & $\begin{array}{l}\text { Cereals } \\
\geq \text { Median }=1 \\
<\text { Median }=0\end{array}$ & $\begin{array}{l}\text { Whole grains } \\
(0-10)\end{array}$ & $\begin{array}{l}\text { Whole grains } \\
\geq \text { Median }=1 \\
<\text { Median }=0\end{array}$ & & $\begin{array}{l}\text { Whole grains } \\
(0-10)\end{array}$ \\
\hline
\end{tabular}

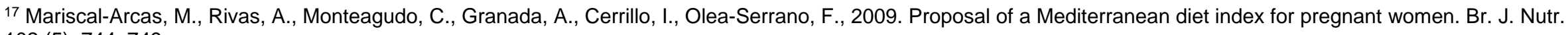
$102(5), 744 \mathrm{e} 749$.

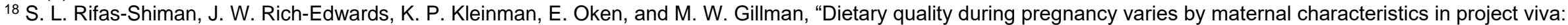
A US cohort," Journal of the American Dietetic Association, vol. 109, no. 6, pp. 1004-1011, 2009.

19 Trichopoulou A, Costacou T, Bamia C, et al. (2003) Adherence to a Mediterranean diet and survival in a Greek population. N Engl J Med 348, $2599-2608$.

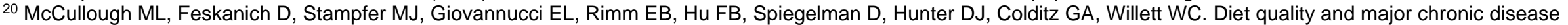
risk in men and women: Moving toward improved dietary guidance. Am J Clin Nutr. 2002; 76:1261-1271.

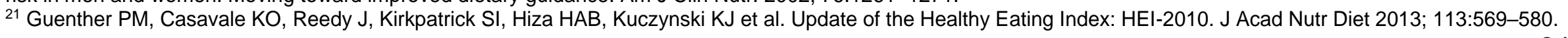




\begin{tabular}{|c|c|c|c|c|c|}
\hline $\begin{array}{l}\text { Index/Score } \\
\text { (Reference) }\end{array}$ & $\begin{array}{l}\text { Mediterranean } \\
\text { Diet Score for } \\
\text { Pregnancy }{ }^{17}\end{array}$ & $\begin{array}{l}\text { AHEI-P } \\
\text { (min-max } \\
{\text { score })^{18}} \\
\end{array}$ & $\begin{array}{l}\text { Alternate } \\
\text { Mediterranean } \\
\text { Diet }^{19}\end{array}$ & $\begin{array}{l}\text { AHEI-P } \\
\text { (min-max } \\
\text { score) }{ }^{20} \\
\end{array}$ & HEI-2010 21 \\
\hline & & & & & $\begin{array}{l}\text { Refined grains } \\
(0-10) \\
\text { (reverse scored) }\end{array}$ \\
\hline \multirow[t]{2}{*}{$\begin{array}{l}\text { Nuts and } \\
\text { legumes }\end{array}$} & $\begin{array}{l}\text { Pulses } \\
\geq \text { Median }=1 ; \\
<\text { Median }=0\end{array}$ & \multirow[t]{2}{*}{$\begin{array}{l}\text { Nuts and legumes } \\
(0-10)\end{array}$} & $\begin{array}{l}\text { Legumes } \\
\geq \text { Median }=1 ; \\
<\text { Median }=0\end{array}$ & & \multirow[t]{2}{*}{$\begin{array}{l}\text { See fish and } \\
\text { other protein } \\
\text { foods below }\end{array}$} \\
\hline & $\begin{array}{l}\text { Nuts included } \\
\text { with fruits }\end{array}$ & & $\begin{array}{l}\text { Nuts } \\
\geq \text { Median }=1 \\
<\text { Median }=0\end{array}$ & & \\
\hline Meat & $\begin{array}{l}\text { Meat } \\
\geq \text { Median = } 0 \\
<\text { Median }=1 \\
\text { (reverse scored) }\end{array}$ & $\begin{array}{l}\text { Red/processed } \\
\text { meat }(10-0)^{4} \\
\text { (reverse scored) }\end{array}$ & $\begin{array}{l}\text { Red and } \\
\text { processed meats } \\
\geq \text { Median = } 0 \\
<\text { Median }=1 \\
\text { (reverse scored) }\end{array}$ & $\begin{array}{l}\text { Ratio of white } \\
\text { (poultry/ fish) to } \\
\text { red meat (beef, } \\
\text { pork, lamb, } \\
\text { processed) } \\
(0-10) \\
\text { Excluded nuts } \\
\text { and soy }\end{array}$ & \\
\hline \multirow[t]{2}{*}{$\begin{array}{l}\text { Fish and other } \\
\text { protein foods }\end{array}$} & \multirow[t]{2}{*}{$\begin{array}{l}\text { Fish } \\
\geq \text { Median = } 1 \\
<\text { Median = }\end{array}$} & & \multirow[t]{2}{*}{$\begin{array}{l}\text { Fish } \\
\geq \text { Median = } 1 \\
<\text { Median }=0\end{array}$} & \multirow[t]{2}{*}{$\begin{array}{l}\text { Included with } \\
\text { meat }\end{array}$} & $\begin{array}{l}\text { Total protein } \\
\text { foods } \\
(0-5)\end{array}$ \\
\hline & & & & & $\begin{array}{l}\text { Seafood and } \\
\text { plant proteins } \\
(0-5)\end{array}$ \\
\hline Dairy & $\begin{array}{l}\text { Dairy products } \\
\geq \text { Median = 0; } \\
<\text { Median = } 1 \\
\text { (reverse scored) }\end{array}$ & & & & $\begin{array}{l}\text { Dairy } \\
(0-10)\end{array}$ \\
\hline Fat & $\begin{array}{l}\text { MUFA:SFA ratio } \\
\geq \text { Median }=1 ; \\
<\text { Median }=0\end{array}$ & $\begin{array}{l}\text { trans fat } \\
(10-0)^{4} \\
\text { (reverse scored) }\end{array}$ & $\begin{array}{l}\text { MUFA:SFA ratio } \\
\geq \text { Median }=1 \\
<\text { Median }=0\end{array}$ & $\begin{array}{l}\text { trans fat } \\
(0-10) \\
\text { (reverse scored) }\end{array}$ & $\begin{array}{l}\text { Fatty acids } \\
(0-10)\end{array}$ \\
\hline
\end{tabular}




\begin{tabular}{|c|c|c|c|c|c|}
\hline $\begin{array}{l}\text { Index/Score } \\
\text { (Reference) }\end{array}$ & $\begin{array}{l}\text { Mediterranean } \\
\text { Diet Score for } \\
\text { Pregnancy }^{17}\end{array}$ & $\begin{array}{l}\text { AHEl-P } \\
\text { (min-max } \\
\text { score) }^{18}\end{array}$ & $\begin{array}{l}\text { Alternate } \\
\text { Mediterranean } \\
\text { Diet }^{19}\end{array}$ & $\begin{array}{l}\text { AHEI-P } \\
\text { (min-max } \\
\text { score) }{ }^{20}\end{array}$ & HEI-2010 21 \\
\hline & & $\begin{array}{l}\text { Long-chain }(n=3) \\
\text { fats }(E P A+D H A)\end{array}$ & & $\begin{array}{l}\text { PUFA:SFA ratio } \\
(0-10)\end{array}$ & \\
\hline & & $(0-10)$ & & & \\
\hline & & $\begin{array}{l}\text { PUFA } \\
(0-10)\end{array}$ & & & \\
\hline Empty calories & & & & & $\begin{array}{l}\text { Empty calories } \\
(0-20)\end{array}$ \\
\hline Fiber & & & & $\begin{array}{l}\text { Fiber } \\
(0-10)\end{array}$ & \\
\hline \multirow[t]{4}{*}{ Micronutrients } & $\begin{array}{l}\text { Folic acid } \\
\geq 2 / 3 \text { RDI for } \\
\text { pregnancy }=1 ; \\
<2 / 3 \text { RDI for } \\
\text { pregnancy }=0\end{array}$ & $\begin{array}{l}\text { Sodium } \\
(10-1)^{4} \\
\text { (reverse scored) }\end{array}$ & & $\begin{array}{l}\text { Folate } \\
(0-10)\end{array}$ & $\begin{array}{l}\text { Sodium } \\
(0-10) \\
\text { (reverse scored) }\end{array}$ \\
\hline & $\begin{array}{l}\text { Iron } \\
\geq 2 / 3 \text { RDI for } \\
\text { pregnancy }=1 \text {; } \\
<2 / 3 \text { RDI for } \\
\text { pregnancy }=0\end{array}$ & $\begin{array}{l}\text { Calcium } \\
(0-10)\end{array}$ & & $\begin{array}{l}\text { Calcium } \\
(0-10)\end{array}$ & \\
\hline & $\begin{array}{l}\text { Calcium } \\
\geq 2 / 3 \text { RDI for } \\
\text { pregnancy }=1 \text {; } \\
<2 / 3 \text { RDI for } \\
\text { pregnancy }=0\end{array}$ & $\begin{array}{l}\text { Folate } \\
(0-10)\end{array}$ & & $\begin{array}{l}\text { Iron } \\
(0-10)\end{array}$ & \\
\hline & & $\begin{array}{l}\text { Iron } \\
(0-10)\end{array}$ & & & \\
\hline $\begin{array}{l}\text { Sweetened } \\
\text { beverages }\end{array}$ & & $\begin{array}{l}\text { Sugar-sweetened } \\
\text { beverages } \\
(10-0)^{4} \\
\text { (reverse scored) }\end{array}$ & & & \\
\hline
\end{tabular}




\begin{tabular}{|c|c|c|c|c|c|}
\hline $\begin{array}{l}\text { Index/Score } \\
\text { (Reference) }\end{array}$ & $\begin{array}{l}\text { Mediterranean } \\
\text { Diet Score for } \\
\text { Pregnancy }^{17}\end{array}$ & $\begin{array}{l}\text { AHEl-P } \\
\text { (min-max } \\
\text { score) }{ }^{18}\end{array}$ & $\begin{array}{l}\text { Alternate } \\
\text { Mediterranean } \\
\text { Diet }^{19}\end{array}$ & $\begin{array}{l}\text { AHEl-P } \\
\text { (min-max } \\
\text { score) }\end{array}$ & HEI-2010 21 \\
\hline \multicolumn{6}{|l|}{ Water } \\
\hline Alcohol & & Excluded alcohol & Excluded alcohol & Excluded alcohol & Excluded alcohol \\
\hline
\end{tabular}


Table 3: Summary of dietary patterns identified using factor or principal components analysis

\begin{tabular}{|c|c|}
\hline Study & Dietary Patterns \\
\hline \multirow[t]{4}{*}{$\begin{array}{l}\text { Bouwland-Both, } \\
2013\end{array}$} & $\begin{array}{l}\text { Energy-rich pattern: high intakes of bread/breakfast cereals, margarine, nuts, snacks/sweets and } \\
\text { nonsweetened nonalcoholic beverages; low intakes of sweetened, nonalcoholic beverages }\end{array}$ \\
\hline & $\begin{array}{l}\text { Mediterranean pattern*: high intakes of vegetables, legumes, pasta/rice, dairy, fish/shellfish, } \\
\text { vegetable oils, alcohol, nonsweetened nonalcoholic beverages; low intakes of processed meat }\end{array}$ \\
\hline & $\begin{array}{l}\text { Western pattern*: high intakes of potatoes, pasta/rice, dairy, fresh meat, processed meat, } \\
\text { margarine and alcohol; low intakes of nuts, fish/shellfish }\end{array}$ \\
\hline & $\begin{array}{l}\text { *Mediterranean and Western dietary patterns showed no significant association with crown-rump } \\
\text { length }(C R L) \text { and so were not included in the analysis }\end{array}$ \\
\hline \multirow[t]{3}{*}{ Chia, 2016} & $\begin{array}{l}\text { Vegetable, fruit, and white rice pattern: characterized by higher intakes of vegetables, fruits, } \\
\text { plain white rice, whole-grain bread, fish, and nuts and seeds and lower intakes of fried potatoes, } \\
\text { burgers, carbonated and sweetened drinks, and flavored rice }\end{array}$ \\
\hline & $\begin{array}{l}\text { Seafood and noodle pattern: higher intakes of soup, seafood, fish and seafood products, noodles } \\
\text { (flavored and in soup), and low-fat red meat and lower intakes of legumes, ethnic bread, white rice, } \\
\text { and curry-based gravies }\end{array}$ \\
\hline & $\begin{array}{l}\text { Pasta, cheese, and processed meat pattern: characterized by high intakes of pasta-, tomato-, } \\
\text { and cream-based gravies, cheese, and processed meat }\end{array}$ \\
\hline \multirow{7}{*}{$\begin{array}{l}\text { Colón-Ramos, } \\
2015\end{array}$} & Healthy pattern: high positive loadings for vegetables, fruits, non-fried fish and chicken, and water \\
\hline & $\begin{array}{l}\text { Processed pattern: high positive loadings for processed meat, fast food items, snacks sweets, } \\
\text { and soft drinks }\end{array}$ \\
\hline & $\begin{array}{l}\text { Southern pattern: high positive loadings for cooked cereals, peaches, corn fried fish, beans, } \\
\text { greens, pig's feet, neck bones oxtails, tongue, and pork }\end{array}$ \\
\hline & Healthy-processed pattern: combination of these two DPs above \\
\hline & Healthy-southern pattern: combination of these two DPs above \\
\hline & Southern-processed pattern: combination of these two DPs above \\
\hline & Mixed pattern: foods from all other patterns together \\
\hline
\end{tabular}




\begin{tabular}{|c|c|}
\hline Study & Dietary Patterns \\
\hline \multirow[t]{3}{*}{ Grieger, 2014} & High-protein/fruit pattern: fish, meat, chicken, fruit, whole grains \\
\hline & High-fat/sugar/takeaway pattern: takeaway foods, potato chips, refined grains, added sugar \\
\hline & Vegetarian-type pattern: vegetables, whole grains, legumes \\
\hline \multirow[t]{3}{*}{ Knudsen, 2008} & $\begin{array}{l}\text { Western pattern: highest intake of high-fat dairy, refined grains, processed and red meat, animal } \\
\text { fat (butter and lard), potatoes, sweets, beer, coffee, and high-energy drinks; lowest intake of fruits } \\
\text { and vegetables ( } 35 \% \text { of energy intake from fat) }\end{array}$ \\
\hline & $\begin{array}{l}\text { Health conscious pattern: high intake of fruits, vegetables, fish, poultry, breakfast cereals, } \\
\text { vegetable juice, and water; lowest intake of meat and fat of animal origin ( } 25 \% \text { of energy intake } \\
\text { from fat) }\end{array}$ \\
\hline & $\begin{array}{l}\text { Intermediate pattern: high intake of low-fat dairy and fruit juice; consumption of the remaining food } \\
\text { groups in between Western and Health conscious DPs (30\% of energy intake from fat) }\end{array}$ \\
\hline \multirow[t]{6}{*}{ Lu, 2016} & $\begin{array}{l}\text { Cereals, eggs, and Cantonese soups pattern: highest content of staples such as rice, pasta, } \\
\text { porridge, eggs, and Cantonese soups; represents a traditional Cantonese diet }\end{array}$ \\
\hline & Dairy pattern: highest content of dairy \\
\hline & $\begin{array}{l}\text { Fruits, nuts, and Cantonese desserts pattern: highest content of fruits, nuts, and Cantonese } \\
\text { desserts }\end{array}$ \\
\hline & Meats pattern: highest content of red meat and processed meat \\
\hline & Vegetables pattern: highest content of leafy and cruciferous vegetables \\
\hline & $\begin{array}{l}\text { Varied pattern: characterized by relatively high intakes of mixed food, including noodles, bread, } \\
\text { root vegetables, melon vegetables, mushrooms, sea vegetables, bean vegetables, processed } \\
\text { vegetables, poultry, animal organ meat, fish, other seafood, bean products, yoghourt, sweet } \\
\text { beverages, puffed food, confectioneries, and snacks }\end{array}$ \\
\hline
\end{tabular}




\begin{tabular}{|c|c|}
\hline Study & Dietary Patterns \\
\hline \multirow[t]{5}{*}{$\begin{array}{l}\text { Northstone, } \\
2008\end{array}$} & $\begin{array}{l}\text { Health-conscious pattern: high loadings on salad, fruit, rice, pasta, breakfast cereals, fish, eggs, } \\
\text { pulses, fruit juices, poultry and non-white bread }\end{array}$ \\
\hline & $\begin{array}{l}\text { Traditional pattern: high loadings on green vegetables and root vegetables, potatoes, peas and to } \\
\text { some extent red meat and poultry }\end{array}$ \\
\hline & $\begin{array}{l}\text { Processed pattern: high loadings on high-fat processed foods, such as meat pies, sausages and } \\
\text { burgers, fried foods, pizza, chips and crisps }\end{array}$ \\
\hline & $\begin{array}{l}\text { Confectionery pattern: characterized by high intakes of confectionery and other foods with high } \\
\text { sugar content such as chocolate, sweets, biscuits, cakes and other puddings }\end{array}$ \\
\hline & $\begin{array}{l}\text { Vegetarian pattern: loaded highly on meat substitutes, pulses, nuts and herbal tea and high } \\
\text { negative loadings were seen with red meat and poultry }\end{array}$ \\
\hline \multirow[t]{3}{*}{ Okubo, 2012} & $\begin{array}{l}\text { Meat and eggs pattern: high intake of beef and pork, processed meat, chicken, eggs, butter and } \\
\text { dairy products }\end{array}$ \\
\hline & $\begin{array}{l}\text { Wheat products pattern: high intake of bread, confectioneries, fruit and vegetable juice, and soft } \\
\text { drinks }\end{array}$ \\
\hline & $\begin{array}{l}\text { Rice, fish, and vegetables pattern: high intake of rice, potatoes, nuts, pulses, fruits, green and } \\
\text { yellow vegetables, white vegetables, mushrooms, seaweeds, Japanese and Chinese tea, fish, } \\
\text { shellfish, sea products, miso soup and salt-containing seasoning }\end{array}$ \\
\hline
\end{tabular}




\section{Table 4: Time point of exposure assessment}

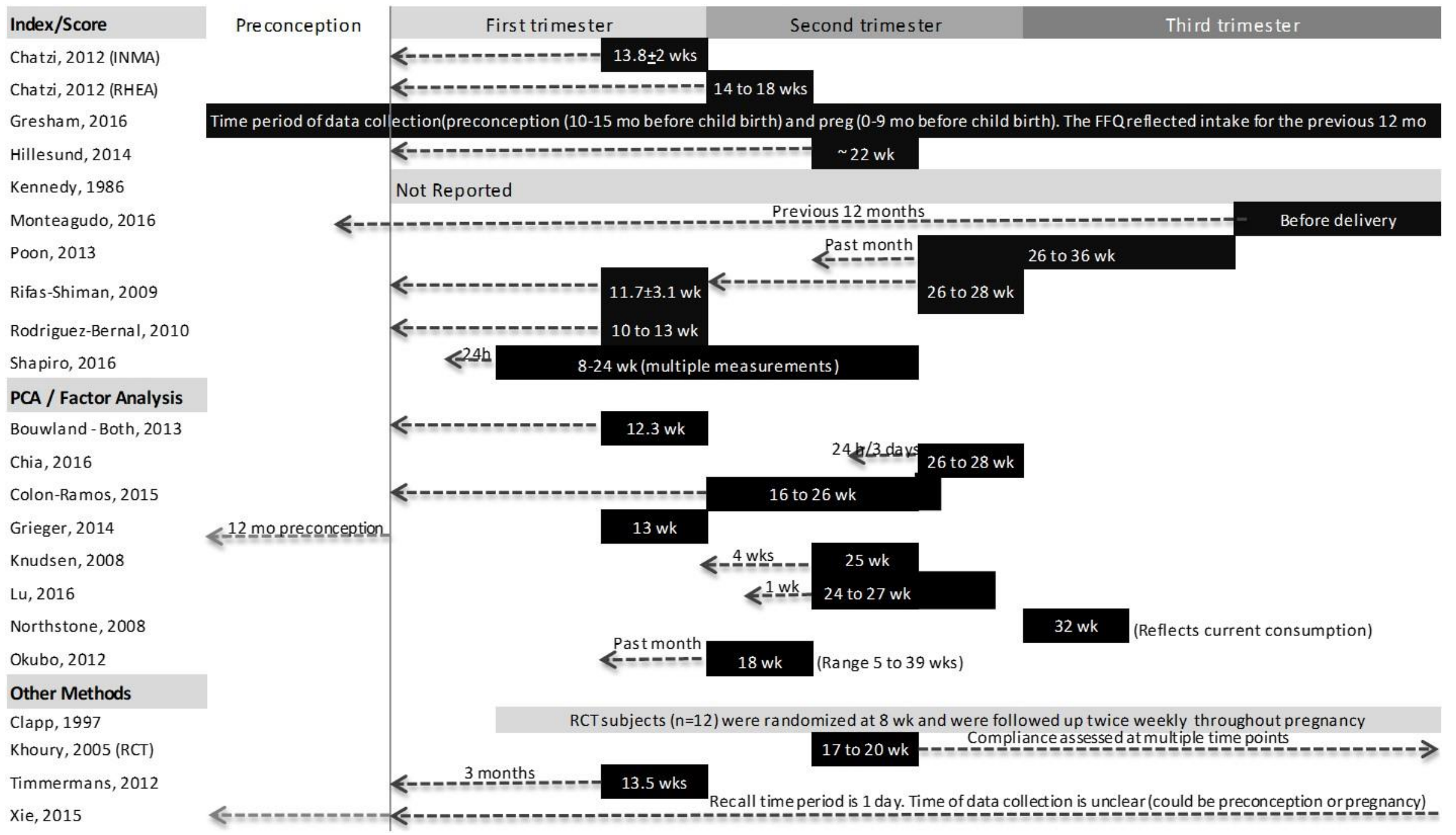


Table 5: Summary of outcome definitions

\begin{tabular}{|c|c|c|c|c|c|c|}
\hline Study & Outcome & $\begin{array}{l}\text { Diagnostic } \\
\text { Criteria }\end{array}$ & $\begin{array}{l}\text { Source of } \\
\text { Criteria }\end{array}$ & $\begin{array}{l}\text { Method of } \\
\text { Assessment }\end{array}$ & References & $\begin{array}{l}\text { Additional } \\
\text { Outcomes } \\
\text { Measured }\end{array}$ \\
\hline \multicolumn{7}{|l|}{ Index/Score } \\
\hline Chatzi, 2012 & $\begin{array}{l}\text { Fetal growth restriction } \\
\text { (weight, length, head } \\
\text { circumference) } \\
\text { Birth weight }\end{array}$ & $\begin{array}{l}\text { FGR: birth weight } \\
<10 \text { th percentile } \\
\text { of the predicted } \\
\text { birth weight } \\
\text { distribution }\end{array}$ & NR & $\begin{array}{l}\text { Obtained from hospital } \\
\text { delivery logs and } \\
\text { medical records }\end{array}$ & N/A & $\begin{array}{l}\text { Birth length } \\
\text { Head } \\
\text { circumference }\end{array}$ \\
\hline Gresham, 2016 & Low birth weight & $\begin{array}{l}\text { LBW: }<2500 \mathrm{~g} \text { or } \\
5.5 \mathrm{lbs}\end{array}$ & NR & Self-reported & $\mathrm{N} / \mathrm{A}$ & $\begin{array}{l}\text { Gestational } \\
\text { hypertension } \\
\text { GDM } \\
\text { Premature birth }\end{array}$ \\
\hline Hillesund, 2014 & $\begin{array}{l}\text { SGA, AGA, LGA } \\
\text { (Gestational age: } \\
\text { 37-42 weeks) }\end{array}$ & $\begin{array}{l}\text { SGA: }<10 \text { th } \\
\text { "gender-specific" } \\
\text { birth weight } \\
\text { percentile } \\
\text { LGA: >90th } \\
\text { "gender- } \\
\text { specific"22 birth } \\
\text { weight percentile }\end{array}$ & NR & $\begin{array}{l}\text { Newborn weight } \\
\text { obtained from Medical } \\
\text { Birth Registry of } \\
\text { Norway and } \\
\text { categorized by } \\
\text { "gender-specific" cut- } \\
\text { off values }\end{array}$ & $\mathrm{N} / \mathrm{A}$ & $\begin{array}{l}\text { Gestational weight } \\
\text { gain }\end{array}$ \\
\hline Kennedy, 1986 & Birth weight & LBW: <2500g & NR & $\begin{array}{l}\text { Extracted from } \\
\text { medical records }\end{array}$ & $\mathrm{N} / \mathrm{A}$ & $\mathrm{N} / \mathrm{A}$ \\
\hline
\end{tabular}

22 "Gender" was used here for consistency with the definition given in the article. 


\begin{tabular}{|c|c|c|c|c|c|c|}
\hline Study & Outcome & $\begin{array}{l}\text { Diagnostic } \\
\text { Criteria }\end{array}$ & $\begin{array}{l}\text { Source of } \\
\text { Criteria }\end{array}$ & $\begin{array}{l}\text { Method of } \\
\text { Assessment }\end{array}$ & References & $\begin{array}{l}\text { Additional } \\
\text { Outcomes } \\
\text { Measured }\end{array}$ \\
\hline $\begin{array}{l}\text { Monteagudo, } \\
2016\end{array}$ & Birth weight (g) & $\mathrm{N} / \mathrm{A}$ & $\mathrm{N} / \mathrm{A}$ & $\begin{array}{l}\text { Measured to the } \\
\text { nearest gram by staff } \\
\text { trained in using } \\
\text { standard protocols } \\
\text { (Lohman, 1988) }\end{array}$ & $\begin{array}{l}\text { Lohman, } \\
1988^{23}\end{array}$ & $\mathrm{~N} / \mathrm{A}$ \\
\hline Poon, 2013 & $\begin{array}{l}\text { SGA } \\
\text { LGA } \\
\text { Birth weight }(\mathrm{g}) \\
\text { (Gestational age: } \\
\geq 35-\leq 43.5\end{array}$ & $\begin{array}{l}\text { SGA: } \leq 10 \text { th } \\
\text { "gender-specific" } \\
\text { birth weight for } \\
\text { gestational age } \\
\text { percentile } \\
\text { LGA: } \geq 90 \text { th } \\
\text { "gender- } \\
\text { specific" } 24 \text { birth } \\
\text { weight for } \\
\text { gestational age } \\
\text { percentile }\end{array}$ & $\begin{array}{l}\text { Canadian } \\
\text { reference for } \\
\text { birth weight for } \\
\text { gestational age }\end{array}$ & $\begin{array}{l}\text { Reported on birth } \\
\text { screener } \\
\text { Gestational age } \\
\text { determined based on } \\
\text { expected date of } \\
\text { delivery and infant } \\
\text { birth date }\end{array}$ & $\begin{array}{l}\text { Kramer, } \\
2001^{25}\end{array}$ & $\begin{array}{l}\text { Early infant growth } \\
\text { (4-6 months) }\end{array}$ \\
\hline $\begin{array}{l}\text { Rifas-Shiman, } \\
2009\end{array}$ & $\begin{array}{l}\text { Birth weight for } \\
\text { gestational age z- } \\
\text { scores } \\
\text { SGA } \\
\text { LGA }\end{array}$ & $\begin{array}{l}\text { SGA: }<10 \text { th birth } \\
\text { weight percentile } \\
\text { LGA: >90th birth } \\
\text { weight percentile }\end{array}$ & Oken, 2003 & NR & $\begin{array}{l}\text { Oken, } \\
2003^{26}\end{array}$ & $\begin{array}{l}\text { Preeclampsia } \\
\text { Gestational } \\
\text { diabetes } \\
\text { Gestational weight } \\
\text { gain }\end{array}$ \\
\hline
\end{tabular}

${ }^{23}$ Lohman, T., Roche, A., Martorell, R., 1988. Anthropometric Standardization Reference Manual. Humana Kinetics Books, Illinois.

24 "Gender" was used here for consistency with the definition given in the article.

${ }^{25}$ M. S.Kramer, R.W. Platt, S.W.Wenet al., "Anewand improved population-based Canadian reference for birth weight for gestational age," Pediatrics, vol. 108, no. 2, article E35, 2001.

${ }^{26}$ Oken E, Kleinman KP, Rich-Edwards JW, Gillman MW. A nearly continuous measure of birth weight for gestational age using a United States national reference. BMC Pediatr. 2003; 3:6. 


\begin{tabular}{|c|c|c|c|c|c|c|}
\hline Study & Outcome & $\begin{array}{l}\text { Diagnostic } \\
\text { Criteria }\end{array}$ & $\begin{array}{l}\text { Source of } \\
\text { Criteria }\end{array}$ & $\begin{array}{l}\text { Method of } \\
\text { Assessment }\end{array}$ & References & $\begin{array}{l}\text { Additional } \\
\text { Outcomes } \\
\text { Measured }\end{array}$ \\
\hline $\begin{array}{l}\text { Rodriguez- } \\
\text { Bernal, } 2010\end{array}$ & $\begin{array}{l}\text { Fetal growth restriction } \\
\text { Birth weight for } \\
\text { gestational age }(g)\end{array}$ & $\begin{array}{l}\text { FGR: below lower } \\
\text { limit of } \\
\text { confidence } \\
\text { interval for growth } \\
\text { potential } \\
\text { predictions at } \\
80 \%\end{array}$ & Mamelle, 2006 & $\begin{array}{l}\text { FGR } \\
\text { Estimated based on } \\
\text { constitutional growth } \\
\text { potential } \\
\text { Birth weight } \\
\text { Obtained from hospital } \\
\text { delivery logs and } \\
\text { medical records } \\
\text { Gestational age based } \\
\text { on date of last } \\
\text { menstrual period self- } \\
\text { reported by the } \\
\text { mothers and corrected } \\
\text { by ultrasound }\end{array}$ & $\begin{array}{l}\text { Mamelle, } \\
2006^{27}\end{array}$ & $\begin{array}{l}\text { Birth length } \\
\text { Head } \\
\text { circumference }\end{array}$ \\
\hline
\end{tabular}

${ }^{27}$ Mamelle N, Boniol M, Rivie're O, et al. Identification of newborns with fetal growth restriction (FGR) in weight and/or length based on constitutional growth potential. Eur J Pediatr 2006;165:717-25. 


\begin{tabular}{|c|c|c|c|c|c|c|}
\hline Study & Outcome & $\begin{array}{l}\text { Diagnostic } \\
\text { Criteria }\end{array}$ & $\begin{array}{l}\text { Source of } \\
\text { Criteria }\end{array}$ & $\begin{array}{l}\text { Method of } \\
\text { Assessment }\end{array}$ & References & $\begin{array}{l}\text { Additional } \\
\text { Outcomes } \\
\text { Measured }\end{array}$ \\
\hline Shapiro, 2016 & $\begin{array}{l}\text { Birth weight }(\mathrm{g}) \\
\text { (Gestational age: } \\
\geq 32 \text { weeks) }\end{array}$ & $\mathrm{N} / \mathrm{A}$ & $\mathrm{N} / \mathrm{A}$ & $\begin{array}{l}\text { Results extracted from } \\
\text { medical records }\end{array}$ & $N / A$ & $\begin{array}{l}\text { Birth length } \\
\text { Head } \\
\text { circumference } \\
\text { Skin-fold thickness } \\
\text { Neonatal body } \\
\text { composition: fat } \\
\text { mass and fat-free } \\
\text { mass }\end{array}$ \\
\hline \multicolumn{7}{|c|}{ PCA / Factor analysis } \\
\hline $\begin{array}{l}\text { Bouwland- } \\
\text { Both, } 2013\end{array}$ & $\begin{array}{l}\text { Crown-rump length } \\
\text { Estimated fetal weight } \\
\text { Birth weight }(\mathrm{g})\end{array}$ & N/A & $\mathrm{N} / \mathrm{A}$ & $\begin{array}{l}\frac{\mathrm{CRL}}{\text { Ultrasound }} \\
\frac{\text { EFW }}{\text { Calculated using }} \\
\text { Hadlock's formula } \\
\text { Birth weight } \\
\text { Obtained from } \\
\text { community midwife } \\
\text { and hospital registries }\end{array}$ & $\begin{array}{l}\text { Usher, } \\
1969^{28} \\
\text { (standards } \\
\text { for } \\
\text { calculating } \\
\text { GA \& BW } \\
\end{array}$ & $\begin{array}{l}\text { Biomarkers of the } \\
\text { one-carbon } \\
\text { pathway (folate } \\
\text { and total } \\
\text { homocysteine in } \\
\text { plasma and } \\
\text { vitamin B12 in } \\
\text { serum) }\end{array}$ \\
\hline
\end{tabular}

${ }^{28}$ Usher R, McLean F. Intrauterine growth of live-born Caucasian infants at sea level: standards obtained from measurements in 7 dimensions of infants born between 25 and 44 weeks of gestation. J Pediatr 1969;74:901-10. 


\begin{tabular}{|c|c|c|c|c|c|}
\hline Study & Outcome & $\begin{array}{l}\text { Diagnostic } \\
\text { Criteria }\end{array}$ & $\begin{array}{l}\text { Source of } \\
\text { Criteria }\end{array}$ & $\begin{array}{l}\text { Method of } \\
\text { Assessment }\end{array}$ & $\begin{array}{l}\text { Additional } \\
\text { Outcomes } \\
\text { Measured }\end{array}$ \\
\hline Chia, 2016 & $\begin{array}{l}\text { Birth weight }(g) S G A \\
\text { LGA }\end{array}$ & $\begin{array}{l}\text { SGA: <10th } \\
\text { percentile for } \\
\text { gestational age } \\
\text { (At } 40 \text { weeks of } \\
\text { gestation, } 2964 \mathrm{~g} \\
\text { for males, } 2947 \mathrm{~g} \\
\text { for females) } \\
\text { LGA: >90th } \\
\text { percentile for } \\
\text { gestational age } \\
\text { (At } 40 \text { weeks of } \\
\text { gestation, } 3838 \mathrm{~g} \\
\text { for males; } 3716 \mathrm{~g} \\
\text { for females) }\end{array}$ & $\begin{array}{l}\text { Mikolajczyk, } \\
2011\end{array}$ & $\begin{array}{l}\text { Birth weight measured Mikolajczyk, } \\
\text { using a SECA } 334 \\
\text { weighing scale to the } \\
\text { nearest } 1 \mathrm{~g} \text {; retrieved } \\
\text { from birth delivery } \\
\text { reports } \\
\text { SGA and LGA } \\
\text { determined using } \\
\text { global birth-weight } \\
\text { reference }\end{array}$ & Preterm birth \\
\hline $\begin{array}{l}\text { Colon-Ramos, } \\
2015\end{array}$ & $\begin{array}{l}\text { Birth weight (weight- } \\
\text { for-length and weight- } \\
\text { for-age } z \text {-scores) } \\
\text { (Gestational age } \geq 37 \\
\text { weeks) }\end{array}$ & $N / A$ & $\mathrm{~N} / \mathrm{A}$ & $\begin{array}{l}\text { Obtained from medical N/A } \\
\text { charts }\end{array}$ & $\begin{array}{l}\text { Head } \\
\text { circumference }\end{array}$ \\
\hline
\end{tabular}

${ }^{29}$ Mikolajczyk RT, Zhang J, Betran AP, Souza JP, Mori R, Gu“Imezoglu AM, Merialdi M. A global reference for fetal-weight and birthweight percentiles. Lancet 2011;377:1855-61. 


\begin{tabular}{|c|c|c|c|c|c|c|}
\hline Study & Outcome & $\begin{array}{l}\text { Diagnostic } \\
\text { Criteria }\end{array}$ & $\begin{array}{l}\text { Source of } \\
\text { Criteria }\end{array}$ & $\begin{array}{l}\text { Method of } \\
\text { Assessment }\end{array}$ & References & $\begin{array}{l}\text { Additional } \\
\text { Outcomes } \\
\text { Measured }\end{array}$ \\
\hline Grieger, 2014 & $\begin{array}{l}\text { Fetal birth weight (g) } \\
\text { (LBW, SGA, IUGR, } \\
\text { LGA, Macrosomia) }\end{array}$ & $\begin{array}{l}\text { LBW: }<2500 \mathrm{~g} \\
\text { and SGA } \\
\text { SGA: <10th } \\
\text { percentile for } \\
\text { gestational age } \\
\text { IUGR: <3rd } \\
\text { percentile for } \\
\text { gestational age } \\
\text { LGA: >90th } \\
\text { percentile for } \\
\text { gestational age } \\
\text { Macrosomia: } \\
>4000 \mathrm{~g}\end{array}$ & NR & $\begin{array}{l}\text { Collected at delivery } \\
\text { (method NR) }\end{array}$ & $\mathrm{N} / \mathrm{A}$ & Preterm birth \\
\hline Knudsen, 2008 & Birth weight (g) (SGA) & $\begin{array}{l}\text { SGA: z-score } \\
\text { below the } 2.5 \text { th } \\
\text { percentile for } \\
\text { respective sex } \\
\text { and gestational } \\
\text { age }\end{array}$ & Marsal, 1996 & $\begin{array}{l}\text { Extracted from the } \\
\text { National Patient } \\
\text { Registry in Denmark }\end{array}$ & $\begin{array}{l}\text { Marsal, } \\
1996^{30}\end{array}$ & $\mathrm{~N} / \mathrm{A}$ \\
\hline
\end{tabular}

${ }^{30}$ Marsal K, Persson PH, Larsen T, Lilja H, Selbing A, Sultan B (1996). Intrauterine growth curves based on ultrasonically estimated foetal weights. Acta Paediatr 85, 843-848. 


\begin{tabular}{|c|c|c|c|c|c|c|}
\hline Study & Outcome & $\begin{array}{l}\text { Diagnostic } \\
\text { Criteria }\end{array}$ & $\begin{array}{l}\text { Source of } \\
\text { Criteria }\end{array}$ & $\begin{array}{l}\text { Method of } \\
\text { Assessment }\end{array}$ & References & $\begin{array}{l}\text { Additional } \\
\text { Outcomes } \\
\text { Measured }\end{array}$ \\
\hline Lu, 2016 & $\begin{array}{l}\text { Birth weight } \\
\text { (SGA, LGA) }\end{array}$ & $\begin{array}{l}\text { SGA: birth weight } \\
<10 \text { th percentile } \\
\text { of Guangzhou } \\
\text { gestational age- } \\
\text { and sex-specific } \\
\text { reference growth } \\
\text { curves } \\
\text { LGA: birth weight } \\
\text { >90th percentile } \\
\text { of Guangzhou } \\
\text { gestational age- } \\
\text { and sex-specific } \\
\text { reference growth } \\
\text { curves }\end{array}$ & $\begin{array}{l}\text { Birthweight } \\
\text { reference in } \\
\text { Guangzhou }\end{array}$ & $\begin{array}{l}\text { Birth weight and } \\
\text { gestational age } \\
\text { obtained from medical } \\
\text { charts }\end{array}$ & $\mathrm{He}, 2014^{31}$ & $\mathrm{~N} / \mathrm{A}$ \\
\hline $\begin{array}{l}\text { Northstone, } \\
2008\end{array}$ & Birth weight (g) & $\mathrm{N} / \mathrm{A}$ & $\mathrm{N} / \mathrm{A}$ & $\begin{array}{l}\text { Recorded in delivery } \\
\text { room; abstracted from } \\
\text { birth notifications }\end{array}$ & $\mathrm{N} / \mathrm{A}$ & $\mathrm{N} / \mathrm{A}$ \\
\hline
\end{tabular}

\footnotetext{
${ }^{31}$ He, J.R.; Xia, H.M.; Liu, Y.; Xia, X.Y.; Mo, W.J.; Wang, P.; Cheng, K.K.; Leung, G.M.; Feng, Q.; Schooling, C.M.; et al. A new birthweight reference in Guangzhou, southern china, and its comparison with the global reference. Arch. Dis. Child. 2014, 99, 1091-1097.
} 


\begin{tabular}{|c|c|c|c|c|c|c|}
\hline Study & Outcome & $\begin{array}{l}\text { Diagnostic } \\
\text { Criteria }\end{array}$ & $\begin{array}{l}\text { Source of } \\
\text { Criteria }\end{array}$ & $\begin{array}{l}\text { Method of } \\
\text { Assessment }\end{array}$ & References & $\begin{array}{l}\text { Additional } \\
\text { Outcomes } \\
\text { Measured }\end{array}$ \\
\hline Okubo, 2012 & $\begin{array}{l}\text { Birth weight (g) } \\
\text { (Gestational age: } \\
37-41 \text { weeks) }\end{array}$ & $\begin{array}{l}\text { SGA: birth } \\
\text { anthropometric } \\
\text { measurements } \\
<10 \text { th percentile } \\
\text { of the Japanese } \\
\text { gestational age- } \\
\text { and sex-specific } \\
\text { reference growth } \\
\text { curves }\end{array}$ & $\begin{array}{l}\text { Ministry of } \\
\text { Health, Labor, } \\
\text { and Welfare or } \\
\text { Japan }\end{array}$ & $\begin{array}{l}\text { Self-administered } \\
\text { questionnaire } \\
\text { (Women were asked } \\
\text { to refer to the neonatal } \\
\text { anthropometric } \\
\text { measurements } \\
\text { recorded by the } \\
\text { obstetrician or midwife } \\
\text { in their Maternal and } \\
\text { Child Health } \\
\text { Handbook) }\end{array}$ & $\begin{array}{l}\text { Ministry of } \\
\text { Health, } \\
\text { Labor, and } \\
\text { I Welfare of } \\
\text { Japan, } \\
2001^{32} \\
\end{array}$ & $\begin{array}{l}\text { Birth length } \\
\text { Head } \\
\text { circumference } \\
\text { Gestational weight } \\
\text { gain }\end{array}$ \\
\hline \multicolumn{7}{|l|}{ Other Method } \\
\hline Clapp, 1997 & Birth weight $(\mathrm{kg})$ & $N / A$ & $N / A$ & $\begin{array}{l}\text { Measured to the } \\
\text { nearest } 10 \mathrm{~g} \text { using a } \\
\text { measurement box } \\
\text { (Clapp, 1996) }\end{array}$ & $\begin{array}{l}\text { Clapp, } \\
1996^{33}\end{array}$ & $\begin{array}{l}\text { Neonatal } \\
\text { Birth length } \\
\text { Head } \\
\text { circumference } \\
\text { Abdominal } \\
\text { circumference } \\
\text { \% body fat } \\
\text { Fat mass (gm) } \\
\text { Lean body mass } \\
\text { (kg) }\end{array}$ \\
\hline
\end{tabular}

\footnotetext{
32 Ministry of Health, Labor, and Welfare of Japan (2001) children and Infant Growth Survey on anthropometric parameters, 2000. http://www.mhlw.go.jp/houdou/0110/h1024-4.html (accessed December 2010).

${ }_{33}$ Clapp JF (1996) Morphometric and neurodevelopmental outcome at age five years of the offspring of women who continued to exercise during pregnancy. J Pediatr 129:856-863
} 


\begin{tabular}{|c|c|c|c|c|c|c|}
\hline Study & Outcome & $\begin{array}{l}\text { Diagnostic } \\
\text { Criteria }\end{array}$ & $\begin{array}{l}\text { Source of } \\
\text { Criteria }\end{array}$ & $\begin{array}{l}\text { Method of } \\
\text { Assessment }\end{array}$ & References & $\begin{array}{l}\text { Additional } \\
\text { Outcomes } \\
\text { Measured }\end{array}$ \\
\hline Khoury, 2005 & $\begin{array}{l}\text { IUGR } \\
\text { Birth weight (g) }\end{array}$ & $\begin{array}{l}\text { IUGR: birth } \\
\text { weight <10th } \\
\text { percentile for } \\
\text { gestational age } \\
\text { and "gender"34 } \\
\text { according to } \\
\text { Norwegian } \\
\text { percentiles }\end{array}$ & $\begin{array}{l}\text { Norwegian } \\
\text { percentiles }\end{array}$ & $\begin{array}{l}\text { Obtained from hospital } \\
\text { records }\end{array}$ & $\begin{array}{l}\text { Skjærven, } \\
2000^{35}\end{array}$ & $\begin{array}{l}\text { Maternal, cord, } \\
\text { and neonatal } \\
\text { cholesterol levels } \\
\text { Hypertensive } \\
\text { complications of } \\
\text { pregnancy } \\
\text { Preterm delivery } \\
\text { Gestational weight } \\
\text { gain }\end{array}$ \\
\hline $\begin{array}{l}\text { Timmermans, } \\
2012\end{array}$ & $\begin{array}{l}\text { Fetal size until birth } \\
\text { (head circumference, } \\
\text { abdominal } \\
\text { circumference, femur } \\
\text { length, estimated fetal } \\
\text { weight) } \\
\text { Placental resistance } \\
\text { (pulsatility index } \\
\text { umbilical artery, } \\
\text { resistance index } \\
\text { uterine artery) } \\
\text { Birth weight }(\mathrm{g})\end{array}$ & $\begin{array}{l}\text { IUGR } 36 \text { : } \\
\text { gestational age- } \\
\text { adjusted birth } \\
\text { weight below } \\
\text { percentile }-2.3 \text { in } \\
\text { the study cohort } \\
(\mathrm{SD}<-2.0)\end{array}$ & NR & $\begin{array}{l}\text { Fetal size (including } \\
\text { IUGR): growth chart } \\
\text { and constructed using } \\
\text { fetal ultrasound } \\
\text { measurements; } \\
\text { Placental resistance: } \\
\text { colour Doppler } \\
\text { (Umbilical artery } \\
\text { pulsatility index and } \\
\text { Uterine artery } \\
\text { resistance index); } \\
\text { Birth weight: medical } \\
\text { records }\end{array}$ & $\mathrm{N} / \mathrm{A}$ & $\mathrm{N} / \mathrm{A}$ \\
\hline
\end{tabular}

\footnotetext{
34 "Gender" was used here for consistency with the definition given in the article.

3511 Skjærven R, Gjessing HK, Bakketeig LS. Birth weight by gestational age in Norway. Acta Obstet Gynecol Scand 2000;79:440-9.

${ }^{36}$ The authors measured IUGR but did not include it in outcome analyses.
} 


\begin{tabular}{|c|c|c|c|c|c|c|}
\hline Study & Outcome & $\begin{array}{l}\text { Diagnostic } \\
\text { Criteria }\end{array}$ & $\begin{array}{l}\text { Source of } \\
\text { Criteria }\end{array}$ & $\begin{array}{l}\text { Method of } \\
\text { Assessment }\end{array}$ & References & $\begin{array}{l}\text { Additional } \\
\text { Outcomes } \\
\text { Measured }\end{array}$ \\
\hline Xie, 2015 & Birth weight $(\mathrm{kg})$ & $N / A$ & $N / A$ & $\begin{array}{l}\text { Self-reported: } \\
\text { Participants who had } \\
\text { given birth were } \\
\text { asked, "How much did } \\
\text { the baby weigh at } \\
\text { birth?" }\end{array}$ & N/A & Gestational age \\
\hline
\end{tabular}




\section{Evidence synthesis}

With 21 included studies, there is a substantial body of evidence available to examine the relationship between dietary patterns before and during pregnancy and gestational age- and sex-adjusted birth weight. Table 6: Results grouped by methodology used for dietary pattern assessment, and the following section provide more information on the findings of each these studies. There is heterogeneity in the methodology employed to define and assess dietary patterns and in how outcomes were reported, which makes it difficult to compare across studies. Further, the time period of dietary assessment varied across studies.

\section{Dietary patterns assessed via index/score}

Nine studies used indices/scores to assess dietary patterns. Four of these studies found an association between adherence to a healthy dietary pattern and birth weight.

- Chatzi et al. (7) assessed maternal adherence to a Mediterranean Diet (MD) characterized by vegetables, legumes, fruits and nuts, cereals, fish and seafood, dairy products, and the ratio of mono- to saturated lipids and all types of meat - in the context of fetal growth restriction and birth weight. The study included data from the multicenter INMA study (INMA-Atlantic and INMAMediterranean) and the RHEA study. Higher MD adherence in INMAMediterranean was associated with a lower risk of fetal growth restriction and higher birth weight. In the other two cohorts, there was no association between $\mathrm{MD}$ adherence and the outcomes.

- Gresham et al. (4) examined the association between adherence to the Australian Recommended Food Score (ARFS) before and during pregnancy and self-reported low birth weight. Components included vegetables, fruit, grain, protein (nuts/bean/soya, meat, fish, eggs), dairy, and fat. The exposure captured dietary intake during the previous 12 months. The time point of data collection ranged from before pregnancy to the end of the third trimester of pregnancy. The study found no significant difference in the odds of low birth weight based on adherence to the ARFS.

- Hillesund et al. (5) assessed maternal adherence to the New Nordic Diet (NND), which measured the frequency of eating the following foods: Nordic fruits (apples, pears, plums, strawberries), root vegetables (carrots, rutabaga and various types of onions), cabbages (kale, cauliflower, broccoli and Brussels sprouts), potatoes, whole grain breads, oatmeal porridge, foods from the wild countryside (wild fish, seafood, game and wild berries), milk and water. (The NND also measured the frequency of main meals per week.) High adherence to the NND, compared to low adherence, was associated with reduced odds of having an SGA baby and greater odds of having an LGA baby.

- Kennedy (18) examined the relationship between a nutritional risk score and birth weight. Components of the risk score included meat or alternatives (meats, fish, poultry, liver, eggs, nuts, peanut butter, legumes), milk and cheese, bread and cereal (whole grain, enriched, other), and four categories of fruits and vegetables (citrus and/or vitamin C-rich vegetables, green and yellow vegetables, all other including potato, and total fruits). The study found no association between nutritional risk score and birth weight.

- Monteagudo et al. (19) assessed the relationship between the Mediterranean Diet Score for Pregnancy (MDS-P) and birth weight. The components of MDS-P 
included vegetables, fruit and nuts, pulses, cereals, fish, ratio of monounsaturated to saturated fats, meat, dairy products and selected micronutrients (folic acid, iron, and calcium). A higher MDS-P score was a predictor of birth weight over 3,500 g.

- Poon et al. (6) assessed adherence to the Alternate Healthy Eating Index for Pregnancy (AHEI-P) and the Alternate Mediterranean Diet (aMED). AHEI-P was characterized by the following components: vegetables, whole fruit, whole grains, nuts and legumes, long-chain (n-3) fats, polyunsaturated fats, folate, calcium, iron, sugar-sweetened beverages, red and processed meat, trans fat, and sodium. aMED was characterized by vegetables, legumes, fruits, nuts, whole grains, fish, and the ratio of monounsaturated to saturated fats and red and processed meats. Adherence to the AHEI-P or aMED was not associated with birth weight or risk of SGA or LGA.

- Rifas-Shiman et al. (9) used an Alternate Healthy Eating Index modified for pregnancy (AHEI-P), which was characterized by intake of vegetables, fruit, ratio of white to red meat, fiber, trans fat, ratio of polyunsaturated to saturated fatty acids, and folate, calcium, and iron from foods. Greater adherence to the AHEI-P during the first or second trimester of pregnancy did not alter the risk of having an SGA or LGA baby.

- Rodriguez-Bernal et al. (8) assessed adherence to the Alternate Healthy Eating Index (AHEI) modified for pregnancy. Components of the AHEI included vegetables, fruit, nuts and soy, ratio of white meat (fish and poultry) to red meat, cereal fiber, trans fat, the ratio of polyunsaturated to saturated fat, and the intake of folate, calcium, and iron from foods. Higher adherence to the AHEI was associated with a lower risk of fetal growth restriction (estimated fetal weight) and improved birth weight.

- Shapiro et al. (10) used the Healthy Eating Index-2010 (HEl-2010) to assess diet quality during pregnancy. The components of HEl-2010 included total fruit, whole fruit, total vegetables, greens and beans, whole grains, dairy, total protein foods, seafood and plant proteins, fatty acids, refined grains, sodium and empty calories. Better maternal diet quality, defined as greater adherence to the HEI2010 (score $>57$ ), was not associated with birth weight.

\section{Dietary patterns assessed via factor or principal component analysis}

Eight studies used data-driven methods (i.e., principal component analysis, exploratory factor analysis, and cluster analysis) to assess dietary patterns. Five of the eight studies found a relationship between diet and birth weight outcomes. A summary of findings from these studies is presented below.

- Bouwland-Both et al. (3) generated three dietary patterns. Higher adherence to the energy-rich dietary pattern (characterized by high intakes of bread/breakfast cereals, margarine, nuts, snacks/sweets and nonsweetened nonalcoholic beverages, and low intakes of sweetened, nonalcoholic beverages) was associated with a greater crown-rump length. However, the energy-rich dietary pattern was not associated with estimated fetal weight or birth weight. The Mediterranean and Western dietary patterns were not associated with crownrump length and so were not included in further analyses.

- Chia et al. (11) showed that greater adherence to a vegetable, fruit and white rice dietary pattern (characterized by higher intakes of vegetables, fruits, plain 
white rice, whole-grain bread, fish, and nuts and seeds and lower intakes of fried potatoes, burgers, carbonated and sweetened drinks, and flavored rice) was associated with an increased risk of having LGA babies, but was not associated with having SGA babies. Chia et al. also assessed other dietary patterns (seafood and noodle and pasta, cheese and processed meat), which were not associated with birth weight or having SGA or LGA babies.

- Colon-Ramos et al. (12) generated three dietary patterns:

- Healthy, characterized by high positive loadings for vegetables, fruits, non-fried fish and chicken, and water

- Processed, characterized by high positive loadings for processed meat, fast food items, snacks sweets, and soft drinks

- Southern, characterized by high positive loadings for cooked cereals, peaches, corn fried fish, beans, greens, pig's feet, neck bones oxtails, tongue, and pork

The authors also generated healthy-processed, healthy-Southern, Southernprocessed, and mixed patterns, reflecting a combination of one or more of the above dietary patterns. None of these patterns were associated with weight-forlength and weight-for-age.

- Grieger et al. (20) measured dietary patterns before pregnancy. Three dietary patterns were generated:

- High protein/fruit, characterized by a diet rich in fish, meat, chicken, fruit and whole grains

- High fat, sugar/takeaway, characterized by takeaway foods, potato chips, refined grains, added sugar

- Vegetarian type, characterized by a diet rich in vegetables, whole grains, legumes

There was no association between any of these dietary patterns and the risk of having LBW or SGA babies.

- Knudsen et al. (13) used PCA to create three different dietary patterns:

- Western pattern, including highest intake of high-fat dairy, refined grains, processed and red meat, animal fat (butter and lard), potatoes, sweets, beer, coffee, and high-energy drinks; lowest intake of fruits and vegetables (35\% of energy intake from fat)

- Health conscious pattern, including high intake of fruits, vegetables, fish, poultry, breakfast cereals, vegetable juice, and water; lowest intake of meat and fat of animal origin (25\% of energy intake from fat)

- Intermediate pattern, including high intake of low-fat dairy and fruit juice; consumption of the remaining food groups in between Western and Health conscious DPs (30\% of energy intake from fat)

When compared to a Western dietary pattern, adherence to an intermediate or health conscious dietary pattern was associated with lower odds of having an SGA baby.

- Lu et al. (14) generated the following dietary patterns using cluster analysis:

- Cereals, eggs, and Cantonese soups pattern, with highest content of staples such as rice, pasta, porridge, eggs, and Cantonese soups; represents a traditional Cantonese diet

- Dairy pattern, with highest content of dairy

- Fruits, nuts, and Cantonese desserts pattern, with highest content of fruits, nuts, and Cantonese desserts 
- Meats pattern, with highest content of red meat and processed meat

- Vegetables pattern, with highest content of leafy and cruciferous vegetables

- Varied pattern, characterized by relatively high intakes of mixed food, including noodles, bread, root vegetables, melon vegetables, mushrooms, sea vegetables, bean vegetables, processed vegetables, poultry, animal organ meat, fish, other seafood, bean products, yoghourt, sweet beverages, puffed food, confectioneries, and snacks

When compared to the cereals, eggs, and Cantonese soups pattern (representing a traditional Cantonese diet), none of the dietary patterns were significantly associated with birth weight for gestational age.

- Northstone et al. (15) used PCA to create the following dietary patterns:

- Health-conscious pattern, with high loadings on salad, fruit, rice, pasta, breakfast cereals, fish, eggs, pulses, fruit juices, poultry and non-white bread

- Traditional pattern, with high loadings on green vegetables and root vegetables, potatoes, peas and to some extent red meat and poultry

- Processed pattern, with high loadings on high-fat processed foods, such as meat pies, sausages and burgers, fried foods, pizza, chips and crisps

- Confectionery pattern, with high intakes of confectionery and other foods with high sugar content such as chocolate, sweets, biscuits, cakes and other puddings

- Vegetarian pattern, loaded highly on meat substitutes, pulses, nuts and herbal tea and high negative loadings of red meat and poultry

The health conscious dietary pattern was associated with higher birth weight, while the processed and vegetarian patterns were associated with lower birth weight. There was no association between the traditional and confectionery patterns and birth weight.

- Okubo et al. (16) used cluster analysis to create the following dietary patterns:

- Meat and eggs pattern, characterized by high intake of beef and pork, processed meat, chicken, eggs, butter and dairy products

- Wheat products pattern, characterized by high intake of bread, confectioneries, fruit and vegetable juice, and soft drinks

- Rice, fish, and vegetables pattern, characterized by high intake of rice, potatoes, nuts, pulses, fruits, green and yellow vegetables, white vegetables, mushrooms, seaweeds, Japanese and Chinese tea, fish, shellfish, sea products, miso soup and salt-containing seasoning Adherence to the wheat products dietary pattern was associated with lower birth weight and a higher risk of SGA when compared to a rice, fish, and vegetable dietary pattern.

\section{Dietary patterns assessed in the RCT}

- Clapp (21) randomly assigned 12 subjects at 8 weeks of gestation to an aboriginal carbohydrate diet (a low glycemic index diet characterized by carbohydrates from unprocessed whole grains, fruits, beans, vegetables, and many dairy products) or a cafeteria carbohydrate diet (a high glycemic index diet characterized by carbohydrates from highly processed grains, root vegetables, and simple sugars). Mothers who were randomized to the cafeteria carbohydrate diet had babies with a greater mean birth weight when compared 
to those randomized to the aboriginal carbohydrate diet.

- Khoury et al. (1) randomly assigned 290 participants to an intervention or control diet. The intervention diet was characterized by higher amounts of fruits and vegetables, fatty fish, vegetable oils, nuts and low-fat dairy. Participants were asked to restrict meat and replace it with avocado, as well as to limit coffee consumption to 2 cups/day. Subjects who were assigned to the control group were asked to consume their usual diet based on Norwegian foodstuffs and not to introduce more oils or low-fat meat and dairy products than usual (1). There were no significant associations between diet and birth weight or risk of IUGR.

\section{Dietary patterns assessed through other methods}

- Timmermans et al. (2) used logistic regression to assess adherence to the Mediterranean dietary pattern, characterized by higher intakes of pasta, rice, vegetable oils, fish, vegetables and alcohol and lower intakes of meat, potatoes and fatty sauces. Compared to high adherence, low adherence was associated with lower fetal abdominal circumference during late pregnancy and lower estimated fetal weight during mid- and late-pregnancy. Similarly, medium and low adherence was associated with lower birth weight when compared to high adherence.

- Xie et al. (17) defined the following dietary patterns on the basis of theoretical expectations:

- High-calorie, sweet pattern, characterized by foods such as doughnuts, ice cream, chocolate candy, regular candy, and cookies

- High-calorie, non-sweet pattern, characterized by steak, fried chicken, fried fish, pizza, hot dogs, sausage, cheese, whole milk, etc.

- Low-calorie pattern, characterized by foods such as low-fat and skim milk, grilled chicken, grilled fish, apples, and breakfast cereal The questionnaire was not validated, and the outcome was self-reported. In this adolescent-only cohort, there was no association between reported dietary patterns and birth weight. 


\begin{tabular}{|l|}
\hline Key for color-coding: \\
\hline Dietary pattern categorized as beneficial when... \\
Greater adherence improves birth weight outcomes \\
Lower adherence is detrimental for birth weight outcomes \\
\hline Dietary pattern categorized as detrimental when... \\
Greater adherence is detrimental for birth weight outcomes \\
Lower adherence improves birth weight outcomes \\
\hline
\end{tabular}

Table 6: Results grouped by methodology used for dietary pattern assessment

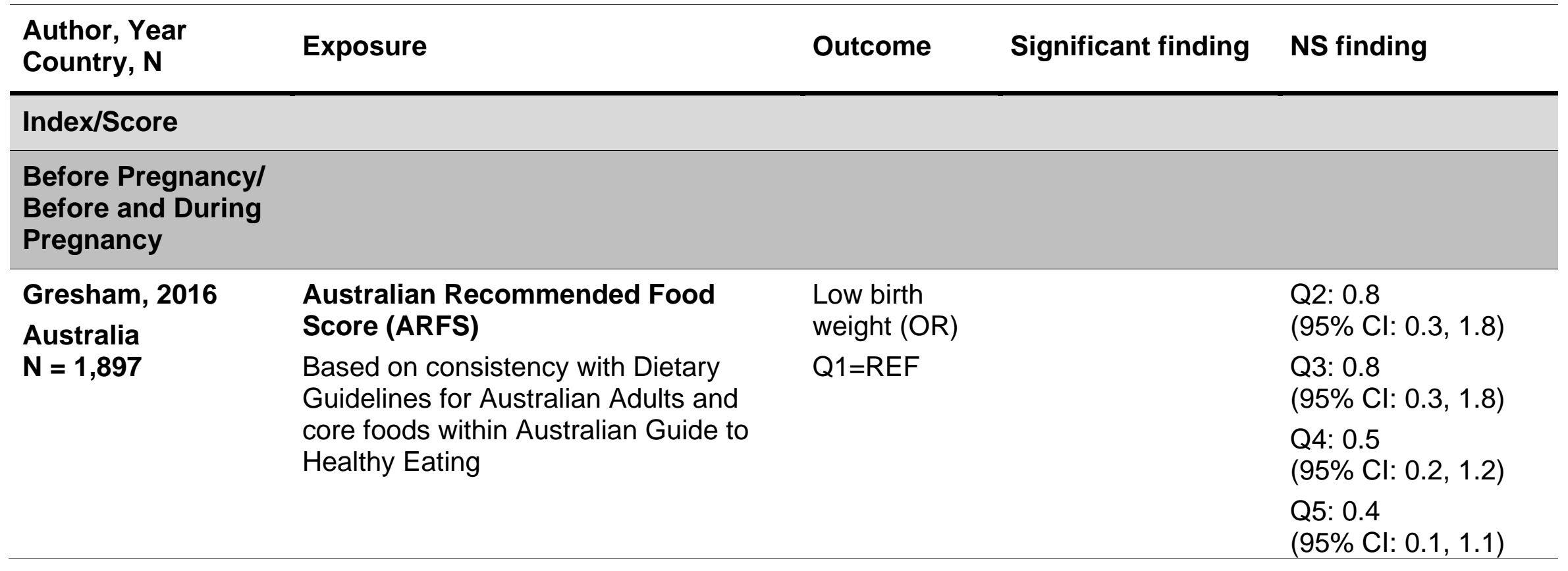




\begin{tabular}{|c|c|c|c|c|}
\hline $\begin{array}{l}\text { Author, Year } \\
\text { Country, N }\end{array}$ & Exposure & Outcome & Significant finding & NS finding \\
\hline $\begin{array}{l}\text { Monteagudo, } 2016 \\
\text { Spain } \\
N=320\end{array}$ & $\begin{array}{l}\text { Mediterranean Diet Score for } \\
\text { Pregnancy (MDS-P) } \\
\text { Positively-scored components: high } \\
\text { consumption (median intake) of } \\
\text { vegetables, fruit and nuts, pulses, } \\
\text { cereals, and fish; a high MUFA:SFA } \\
\text { ratio; low consumption of meat and } \\
\text { dairy products; and the Spanish RDI of } \\
\text { selected micronutrients (folic acid, Fe, } \\
\text { and Ca), considering two-thirds of the } \\
\text { RDI for pregnant women as the cut-off } \\
\text { point }\end{array}$ & $\begin{array}{l}\text { Birth weight } \\
\left(\mathrm{OR}^{*}\right) \\
\text { Lower=REF } \\
\\
{ }^{*} \text { odds of } \\
\text { weighing } \\
>3.5 \mathrm{~kg} \text { (third } \\
\text { tertile) vs } \\
<2.5 \mathrm{~kg} \text { (first } \\
\text { tertile) }\end{array}$ & $\begin{array}{l}\text { Higher: } 6.162 \\
\text { (95\% Cl: } 1.438, \\
26.394)\end{array}$ & \\
\hline
\end{tabular}




\begin{tabular}{|c|c|c|c|c|}
\hline $\begin{array}{l}\text { Author, Year } \\
\text { Country, N }\end{array}$ & Exposure & Outcome & Significant finding & NS finding \\
\hline \multirow{15}{*}{$\begin{array}{l}\text { Chatzi, } 2012 \\
\text { Spain } \\
N=2,461 \\
\text { Greece } \\
N=889\end{array}$} & \multirow{9}{*}{$\begin{array}{l}\text { Mediterranean Diet scale } \\
\text { Positively scored components: } \\
\text { vegetables, legumes, fruits and nuts, } \\
\text { cereals, fish and seafood, dairy } \\
\text { products, and ratio of mono- to } \\
\text { saturated lipids } \\
\text { Negatively scored components: all } \\
\text { types of meat }\end{array}$} & \multirow{9}{*}{$\begin{array}{l}\text { Fetal growth } \\
\text { restriction } \\
(\mathrm{RR}) \\
\text { Low=REF }\end{array}$} & \multirow{9}{*}{$\begin{array}{l}\text { Spain (INMA- } \\
\text { Mediterranean): } \\
\text { High: } 0.50 \\
(95 \% \mathrm{Cl}: 0.28,0.90)\end{array}$} & Spain (INMA- \\
\hline & & & & Mediterranean): \\
\hline & & & & $(95 \%$ Cl: $0.54,1.06)$ \\
\hline & & & & $\begin{array}{l}\text { Spain (INMA- } \\
\text { Atlantic): }\end{array}$ \\
\hline & & & & $\begin{array}{l}\text { Medium: } 1.24 \\
(95 \% \mathrm{Cl}: 0.81,1.89)\end{array}$ \\
\hline & & & & $\begin{array}{l}\text { High: } 0.97 \\
(95 \% \text { Cl: } 0.42,2.26)\end{array}$ \\
\hline & & & & Greece: \\
\hline & & & & $\begin{array}{l}\text { Medium: } 1.82 \\
(95 \% \mathrm{Cl}: 0.95,3.49)\end{array}$ \\
\hline & & & & $\begin{array}{l}\text { High: } 1.96 \\
(95 \% \text { Cl: } 0.90,4.25)\end{array}$ \\
\hline & & \multirow{6}{*}{$\begin{array}{l}\text { Birth weight } \\
(\beta) \\
\text { Low=REF }\end{array}$} & $\begin{array}{l}\text { Spain (INMA- } \\
\text { Mediterranean): }\end{array}$ & $\begin{array}{l}\text { Spain (INMA- } \\
\text { Atlantic): }\end{array}$ \\
\hline & & & $\begin{array}{l}\text { Medium: } 55.20 \mathrm{~g} \\
(\mathrm{SE}=23.52)\end{array}$ & $\begin{array}{l}\text { Medium: }-26.46 \mathrm{~g} \\
(\mathrm{SE}=26.03)\end{array}$ \\
\hline & & & $\begin{array}{l}\text { High: } 87.78 \mathrm{~g} \\
(\mathrm{SE}=33.40)\end{array}$ & $\begin{array}{l}\text { High: }-82.85 \mathrm{~g} \\
(\mathrm{SE}=47.67)\end{array}$ \\
\hline & & & & Greece: \\
\hline & & & & $\begin{array}{l}\text { Medium: }-33.67 \mathrm{~g} \\
(\mathrm{SE}=31.78)\end{array}$ \\
\hline & & & & $\begin{array}{l}\text { High: }-20.42 \mathrm{~g} \\
(\mathrm{SE}=42.33)\end{array}$ \\
\hline
\end{tabular}




\begin{tabular}{|c|c|c|c|c|}
\hline $\begin{array}{l}\text { Author, Year } \\
\text { Country, N }\end{array}$ & Exposure & Outcome & Significant finding & NS finding \\
\hline Hillesund, 2014 & New Nordic Diet score & SGA (OR) & \multirow{13}{*}{$\begin{array}{l}\text { High: } 0.92 \\
(95 \% \text { Cl: } 0.86,0.99)\end{array}$} & \multirow{13}{*}{$\begin{array}{l}\text { Medium: } 0.95 \\
(95 \% \mathrm{Cl}: 0.89,1.02)\end{array}$} \\
\hline \multirow[t]{16}{*}{$\begin{array}{l}\text { Norway } \\
N=66,597\end{array}$} & $\begin{array}{l}\text { Constructed to measure adherence } \\
\text { with the fundamental guidelines of the } \\
\text { NND }\end{array}$ & Low $=$ REF & & \\
\hline & Positively-scored components: (i) & & & \\
\hline & $\begin{array}{l}\text { eating } \geq 24 \text { main meals/week; (ii) eating } \\
\text { Nordic fruits } \geq 5 \text { times/week; (iii) eating }\end{array}$ & & & \\
\hline & root vegetables $\geq 5$ times/week; (iv) & & & \\
\hline & eating cabbage $\geq 2$ times/week; (v) & & & \\
\hline & eating potatoes $\geq$ one-third of total & & & \\
\hline & occasions of eating potatoes, rice or & & & \\
\hline & more often than refined bread; (vii) & & & \\
\hline & eating oatmeal $\geq$ monthly; (viii) eating & & & \\
\hline & fish/game/berries about 2 times/week; & & & \\
\hline & (ix) drinking milk more often than juice; & & & \\
\hline & $\begin{array}{l}\text { and }(x) \text { drinking } \geq 6 \text { times as much } \\
\text { water as sugar-sweetened beverages }\end{array}$ & & & \\
\hline & & \multicolumn{2}{|l|}{ AGA (OR) } & Medium: 1.00 (REF) \\
\hline & & \multicolumn{2}{|l|}{ Low=REF } & High: 1.00 (REF) \\
\hline & & LGA (OR) & \multirow{2}{*}{$\begin{array}{l}\text { High: } 1.07 \\
(95 \% \text { Cl: } 1.00,1.15)\end{array}$} & \multirow{2}{*}{$\begin{array}{l}\text { Medium: } 1.04 \\
\text { (95\% Cl: } 0.97,1.12)\end{array}$} \\
\hline & & Low $=$ REF & & \\
\hline
\end{tabular}




\begin{tabular}{|c|c|c|c|c|}
\hline $\begin{array}{l}\text { Author, Year } \\
\text { Country, N }\end{array}$ & Exposure & Outcome & Significant finding & NS finding \\
\hline \multirow{6}{*}{$\begin{array}{l}\text { Kennedy, } 1986 \\
\text { USA } \\
\mathbf{N}=886\end{array}$} & Nutritional Risk score & \multirow{3}{*}{$\begin{array}{l}\text { Birth weight } \\
\text { (mean) }\end{array}$} & & High risk: 3,273g \\
\hline & $\begin{array}{l}\text { Categorized as high, moderate, or low } \\
\text { risk }\end{array}$ & & & $\begin{array}{l}\text { Moderate risk: } \\
3,262 \mathrm{~g}\end{array}$ \\
\hline & \multirow[t]{4}{*}{$\begin{array}{l}\text { Positively-scored components: meat or } \\
\text { alternatives (meats, fish, poultry [fresh } \\
\text { or processed], liver, eggs, nuts, peanut } \\
\text { butter, legumes), milk and cheese } \\
\text { (maximum of } 3 \text { oz of cheese scored), } \\
\text { bread and cereal (whole grain, } \\
\text { enriched, other), and fruits and } \\
\text { vegetables (4 categories: citrus and/or } \\
\text { vitamin C-rich vegetables, green and } \\
\text { yellow vegetables, all other including } \\
\text { potato, total fruits) }\end{array}$} & & & Low risk: $3,319 \mathrm{~g}$ \\
\hline & & Low birth & & High risk: $7.0 \%$ \\
\hline & & weight (\%) & & Moderate risk: $6.9 \%$ \\
\hline & & & & Low risk: $7.4 \%$ \\
\hline \multirow{4}{*}{$\begin{array}{l}\text { Poon, } 2013 \\
\text { USA } \\
N=893\end{array}$} & \multirow{2}{*}{$\begin{array}{l}\text { Alternative Healthy Eating Index for } \\
\text { Pregnancy (AHEI-P) }\end{array}$} & SGA (RR) & & \multirow{3}{*}{$\begin{array}{l}\text { T2: } 0.73 \\
\text { (95\% Cl: } 0.41,1.31) \\
\text { T3: } 0.93 \\
(95 \% \text { Cl: } 0.49,1.75)\end{array}$} \\
\hline & & \multirow{3}{*}{$\mathrm{T} 1=\mathrm{REF}$} & & \\
\hline & $\begin{array}{l}\text { Positively-scored components: } \\
\text { vegetables, whole fruit, whole grains, } \\
\text { nuts and legumes, long-chain (n-3) fats, } \\
\text { polyunsaturated fats, folate, calcium, } \\
\text { and iron }\end{array}$ & & & \\
\hline & $\begin{array}{l}\text { Negatively-scored components: sugar- } \\
\text { sweetened beverages, red and } \\
\text { processed meat, trans fat, and sodium }\end{array}$ & & & \\
\hline
\end{tabular}




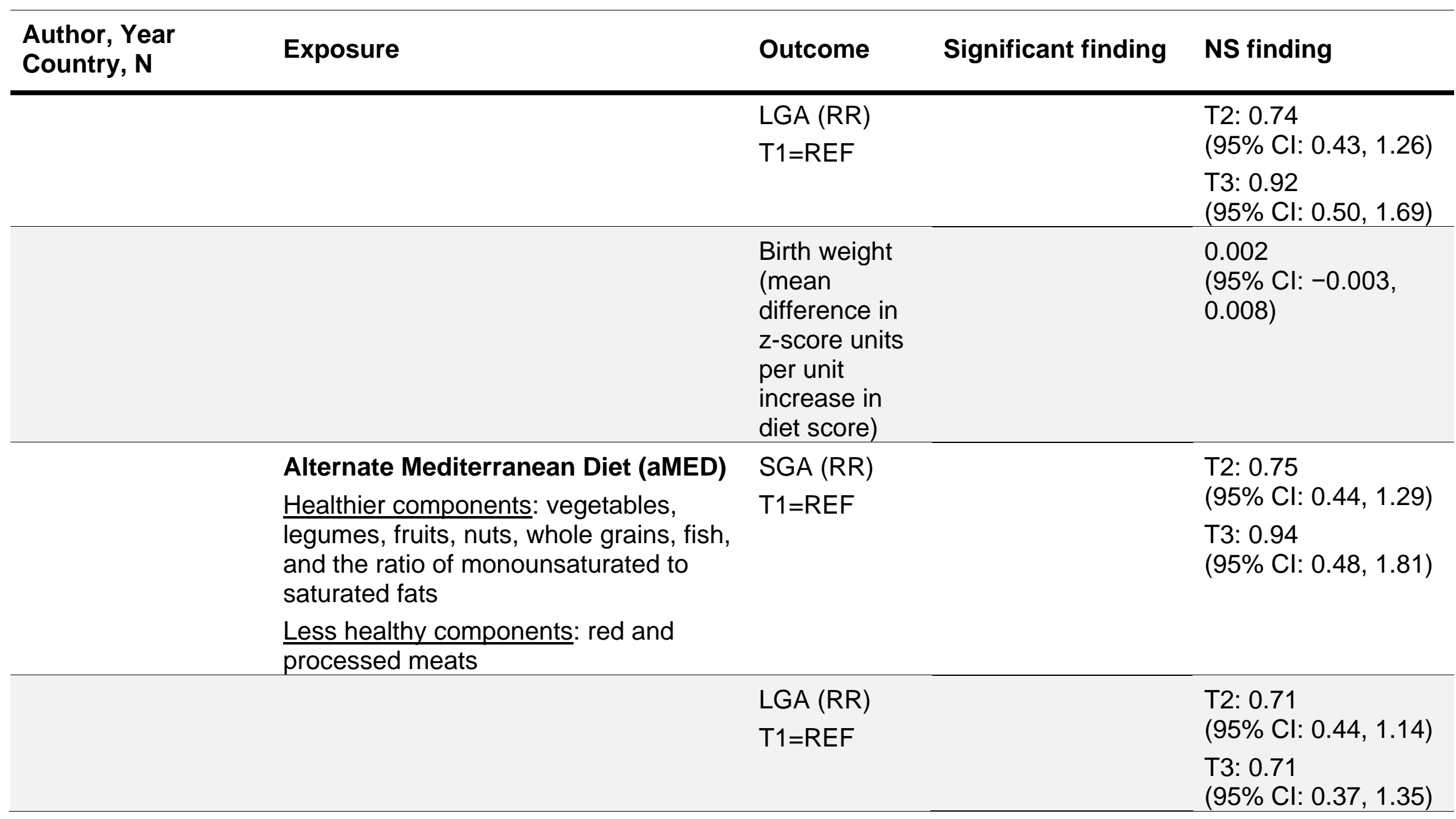




\begin{tabular}{|c|c|c|c|c|}
\hline $\begin{array}{l}\text { Author, Year } \\
\text { Country, N }\end{array}$ & Exposure & Outcome & Significant finding & NS finding \\
\hline & & $\begin{array}{l}\text { Birth weight } \\
\text { (mean } \\
\text { difference in } \\
\text { z-score units } \\
\text { per unit } \\
\text { increase in } \\
\text { diet score) }\end{array}$ & & $\begin{array}{l}-0.003 \\
(95 \% \mathrm{Cl}:-0.036 \\
0.031)\end{array}$ \\
\hline Rifas-Shiman, 2009 & AHEI-P & SGA (OR) & & $1^{\text {st }}$ trimester: \\
\hline \multirow[t]{5}{*}{$\begin{array}{l}\text { USA } \\
N=1,777 \text { (first } \\
\text { trimester) } \\
N=1,666 \text { (second } \\
\text { trimester) }\end{array}$} & $\begin{array}{l}\text { vegetables; fruit; ratio of white to red } \\
\text { meat; fiber; trans fat; ratio of } \\
\text { polyunsaturated to saturated fatty } \\
\text { acids; and folate, calcium, and iron from } \\
\text { foods }\end{array}$ & $A G A=R E F$ & & $\begin{array}{l}0.92 \\
(95 \% \mathrm{Cl}: 0.82,1.02) \\
2^{\text {nd }} \text { trimester: } \\
1.00 \\
(95 \% \mathrm{Cl}: 0.90,1.10)\end{array}$ \\
\hline & & LGA (OR) & & $1^{\text {st }}$ trimester: \\
\hline & & $A G A=R E F$ & & $\begin{array}{l}0.95 \\
(95 \% \mathrm{Cl}: 0.89,1.02)\end{array}$ \\
\hline & & & & $2^{\text {nd }}$ trimester: \\
\hline & & & & $\begin{array}{l}0.99 \\
(95 \% \text { Cl: } 0.92,1.07)\end{array}$ \\
\hline
\end{tabular}




\begin{tabular}{|c|c|c|c|c|}
\hline $\begin{array}{l}\text { Author, Year } \\
\text { Country, N }\end{array}$ & Exposure & Outcome & Significant finding & NS finding \\
\hline $\begin{array}{l}\text { Rodriguez-Bernal, } \\
2010 \\
\text { Spain } \\
N=787\end{array}$ & $\begin{array}{l}\text { AHEI-P } \\
\text { Positively-scored components: } \\
\text { vegetables }(5 \text { serv/d); fruit }(4 \mathrm{serv} / \mathrm{d}) \text {; } \\
\text { nuts and soy }(1 \mathrm{serv} / \mathrm{d}) ; \text { ratio of white to } \\
\text { red meat }(\geq 4: 1) \text {; cereal fiber }(15 \mathrm{~g} / \mathrm{d}) ; \\
\text { trans fat }(\leq 0.5 \% \text { of energy); ratio of } \\
\text { polyunsaturated to saturated fat }(\geq 1) \text {; } \\
\text { and folate }(\geq 600 \mathrm{~g} / \mathrm{d}) \text {, calcium } \\
(\geq 1000 \mathrm{mg} / \mathrm{d}) \text {, and iron }(\geq 27 \mathrm{mg} / \mathrm{d}) \\
\text { intakes from foods }\end{array}$ & $\begin{array}{l}\text { Fetal growth } \\
\text { restriction in } \\
\text { weight (OR) } \\
\text { Q1=REF }\end{array}$ & $\begin{array}{l}\text { Q3: } 0.35 \\
\text { (95\% Cl: } 0.16,0.76) \\
\text { Q4: } 0.51 \\
(95 \% \mathrm{Cl}: 0.26,0.99) \\
\text { Q5: } 0.24 \\
(95 \% \mathrm{Cl}: 0.10,0.55)\end{array}$ & $\begin{array}{l}\text { Q2: } 0.55 \\
(95 \% \mathrm{Cl}: 0.28,1.08)\end{array}$ \\
\hline & & $\begin{array}{l}\text { Birth weight } \\
\text { adjusted for } \\
\text { gestational } \\
\text { age }(\beta) \\
\text { Q1=REF }\end{array}$ & $\begin{array}{l}\text { Q2: } 92.69 \mathrm{~g} \\
(95 \% \mathrm{Cl}: 3.24 \\
182.16) \\
\text { Q4: } 126.25 \mathrm{~g} \\
(95 \% \mathrm{Cl}: 38.53 \text {, } \\
213.96) \\
\text { Q5: } 114.15 \mathrm{~g} \\
(95 \% \mathrm{Cl}: 27.07 \\
201.23)\end{array}$ & $\begin{array}{l}\text { Q3: } 83.45 \mathrm{~g} \\
\text { (95\% Cl: }-7.53 \text {, } \\
\text { 174.43) }\end{array}$ \\
\hline $\begin{array}{l}\text { Shapiro, } 2016 \\
\text { USA } \\
N=1,079\end{array}$ & $\begin{array}{l}\text { Healthy Eating Index-2010 (HEI- } \\
\text { 2010): } \\
\text { Components: total fruit, whole fruit, total } \\
\text { vegetables, greens and beans, whole } \\
\text { grains, dairy, total protein foods, } \\
\text { seafood and plant proteins, fatty acids, } \\
\text { refined grains, sodium and empty } \\
\text { calories }\end{array}$ & $\begin{array}{l}\text { Birth weight } \\
(\beta) \\
\text { Score } \\
>57=\text { REF }\end{array}$ & & $\begin{array}{l}\text { Score } \leq 57: 27.86 \mathrm{~g} \\
(95 \% \mathrm{Cl}:-21.16, \\
76.89)\end{array}$ \\
\hline
\end{tabular}




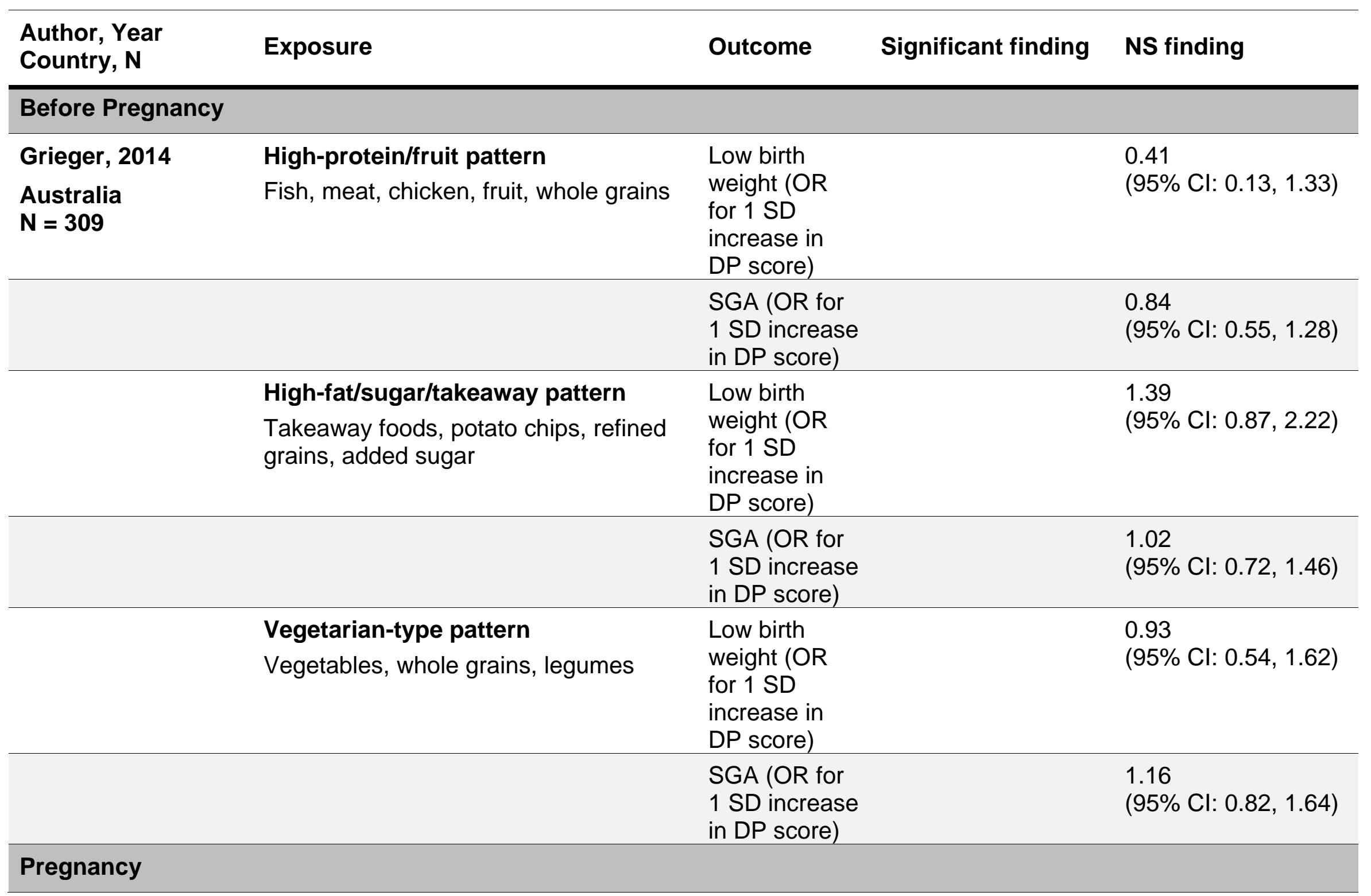




\begin{tabular}{|c|c|c|c|c|}
\hline $\begin{array}{l}\text { Author, Year } \\
\text { Country, N }\end{array}$ & Exposure & Outcome & Significant finding & NS finding \\
\hline \multirow[t]{8}{*}{$\begin{array}{l}\text { Bouwland-Both, } \\
2013 \\
\text { The Netherlands } \\
\mathbf{N}=847\end{array}$} & $\begin{array}{l}\text { Energy-rich pattern } \\
\text { High intakes of bread/breakfast cereals, } \\
\text { margarine, nuts, snacks/sweets and } \\
\text { nonsweetened nonalcoholic beverages; } \\
\text { Low intakes of sweetened, nonalcoholic } \\
\text { beverages }\end{array}$ & $\begin{array}{l}\text { Crown-rump } \\
\text { length } \\
\text { Low=REF }\end{array}$ & $\begin{array}{l}\text { Millimeters: } \\
\text { High: } 1.62 \\
\text { (95\% Ci: } 0.52,2.72) \\
\text { SD score: } \\
\text { High: } 0.23 \\
(95 \% \text { Ci: } 0.08,0.38)\end{array}$ & $\begin{array}{l}\text { Millimeters: } \\
\text { Intermediate: } 0.87 \\
\text { (95\% Cl: }-0.23,1.96) \\
\text { SD score: } \\
\text { Intermediate: } 0.10 \\
(95 \% \mathrm{Cl}:-0.05,0.25)\end{array}$ \\
\hline & & $\begin{array}{l}\text { Estimated } \\
\text { fetal weight } \\
(\beta) \\
\text { Low=REF }\end{array}$ & & $\begin{array}{l}2^{\text {nd }} \text { trimester }-S D \\
\text { score: } \\
\text { Intermediate: }-0.01 \\
(95 \% \mathrm{Cl}:-0.15,0.12)\end{array}$ \\
\hline & & & & $\begin{array}{l}\text { High: } 0.01 \\
(95 \% \text { Cl: }-0.12,0.15)\end{array}$ \\
\hline & & & & $\begin{array}{l}3^{\text {rd }} \text { trimester - SD } \\
\text { score: }\end{array}$ \\
\hline & & & & $\begin{array}{l}\text { Intermediate: } 0.11 \\
(95 \% \mathrm{Cl}:-0.04,0.26)\end{array}$ \\
\hline & & & & $\begin{array}{l}\text { High: } 0.03 \\
(95 \% \text { Cl: }-0.12,0.18)\end{array}$ \\
\hline & & $\begin{array}{l}\text { Birth weight } \\
(\beta) \\
\text { Low=REF }\end{array}$ & & $\begin{array}{l}\text { SD score: } \\
\text { Intermediate: } 0.04 \\
(95 \% \mathrm{Cl}:-0.14,0.22)\end{array}$ \\
\hline & & & & $\begin{array}{l}\text { High: } 0.10 \\
(95 \% \mathrm{Cl}:-0.08,0.28)\end{array}$ \\
\hline
\end{tabular}




\begin{tabular}{|c|c|c|c|c|}
\hline $\begin{array}{l}\text { Author, Year } \\
\text { Country, } \mathbf{N}\end{array}$ & Exposure & Outcome & Significant finding & NS finding \\
\hline & $\begin{array}{l}\text { Mediterranean pattern } \\
\text { High intakes of vegetables, legumes, } \\
\text { pasta/rice, dairy, fish/shellfish, } \\
\text { vegetable oils, alcohol, nonsweetened } \\
\text { nonalcoholic beverages; } \\
\underline{\text { Low intakes of processed meat }}\end{array}$ & $\mathrm{N} / \mathrm{A}$ & $N / A$ & $\begin{array}{l}\text { Pattern showed no } \\
\text { significant } \\
\text { association with } \\
\text { crown-rump length } \\
\text { and so was not } \\
\text { included in the } \\
\text { analysis }\end{array}$ \\
\hline & $\begin{array}{l}\text { Western pattern } \\
\text { High intakes of potatoes, pasta/rice, } \\
\text { dairy, fresh meat, processed meat, } \\
\text { margarine and alcohol; } \\
\underline{\text { Low intakes of nuts, fish/shellfish }}\end{array}$ & $\mathrm{N} / \mathrm{A}$ & $N / A$ & $\begin{array}{l}\text { Pattern showed no } \\
\text { significant } \\
\text { association with } \\
\text { crown-rump length } \\
\text { and so was not } \\
\text { included in the } \\
\text { analysis }\end{array}$ \\
\hline \multirow[t]{3}{*}{$\begin{array}{l}\text { Chia, } 2016 \\
\text { Singapore } \\
N=923\end{array}$} & $\begin{array}{l}\text { Vegetable, fruit, and white rice } \\
\text { pattern } \\
\text { Higher intakes of vegetables, fruits, } \\
\text { plain white rice, whole-grain bread, fish, } \\
\text { and nuts and seeds, and } \\
\text { Lower intakes of fried potatoes, } \\
\text { burgers, carbonated and sweetened } \\
\text { drinks, and flavored rice }\end{array}$ & \multicolumn{2}{|l|}{$\begin{array}{l}\text { Birth weight } \\
(\beta \text { per } 1-S D \\
\text { increase in } \\
\text { dietary } \\
\text { pattern score })\end{array}$} & $\begin{array}{l}29.47 \mathrm{~g} \\
(95 \% \mathrm{Cl}:-0.74 \\
59.67)\end{array}$ \\
\hline & & \multicolumn{2}{|l|}{$\begin{array}{l}\text { SGA (OR) } \\
\text { Low=REF }\end{array}$} & \multirow[t]{2}{*}{$\begin{array}{l}\text { Higher: } 1.03 \\
(95 \% \text { Cl: } 0.82,1.30)\end{array}$} \\
\hline & & $\begin{array}{l}\text { LGA (OR) } \\
\text { Low=REF }\end{array}$ & $\begin{array}{l}\text { Higher: } 1.31 \\
(95 \% \mathrm{Cl}: 1.06,1.62)\end{array}$ & \\
\hline
\end{tabular}




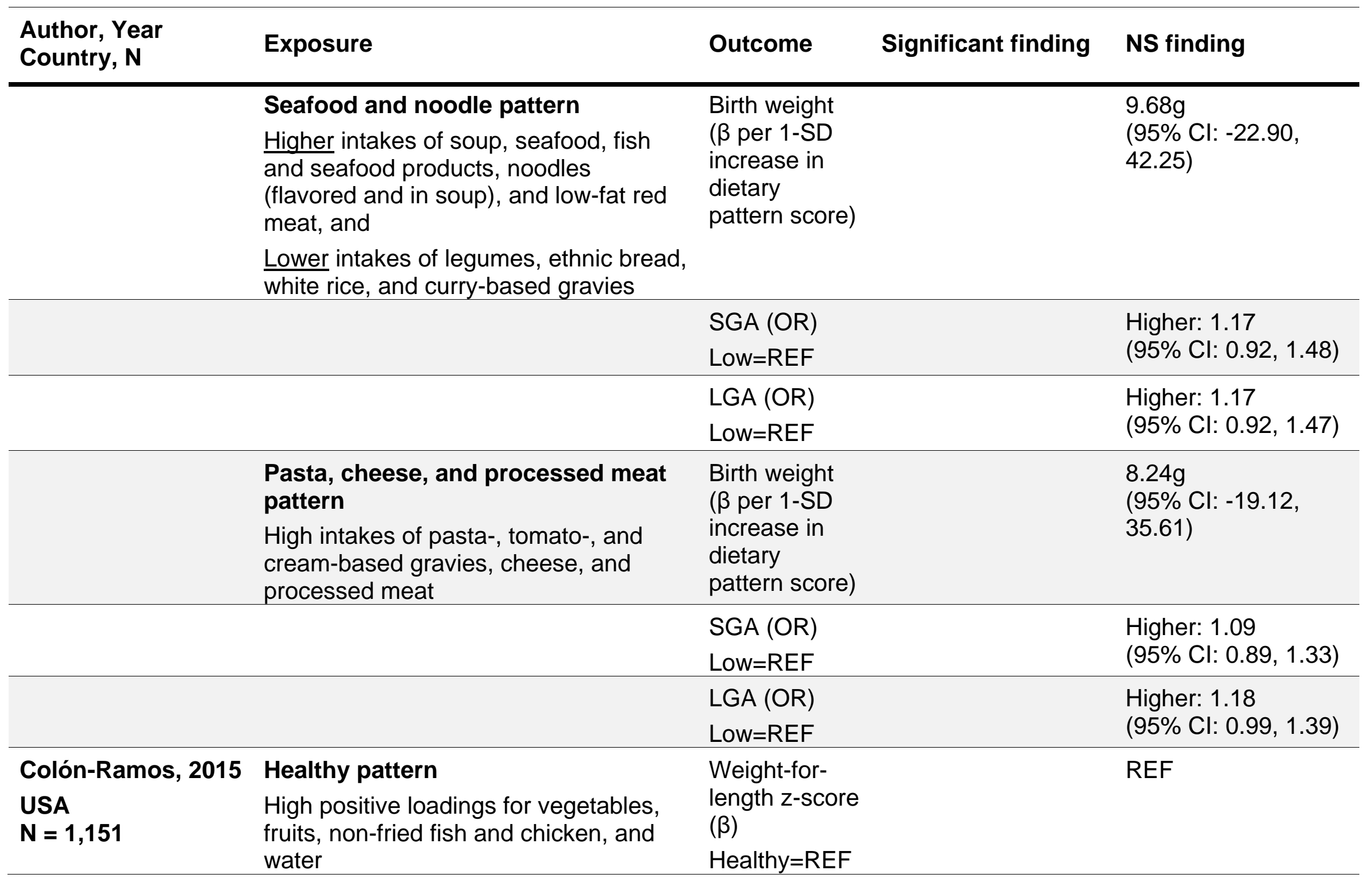




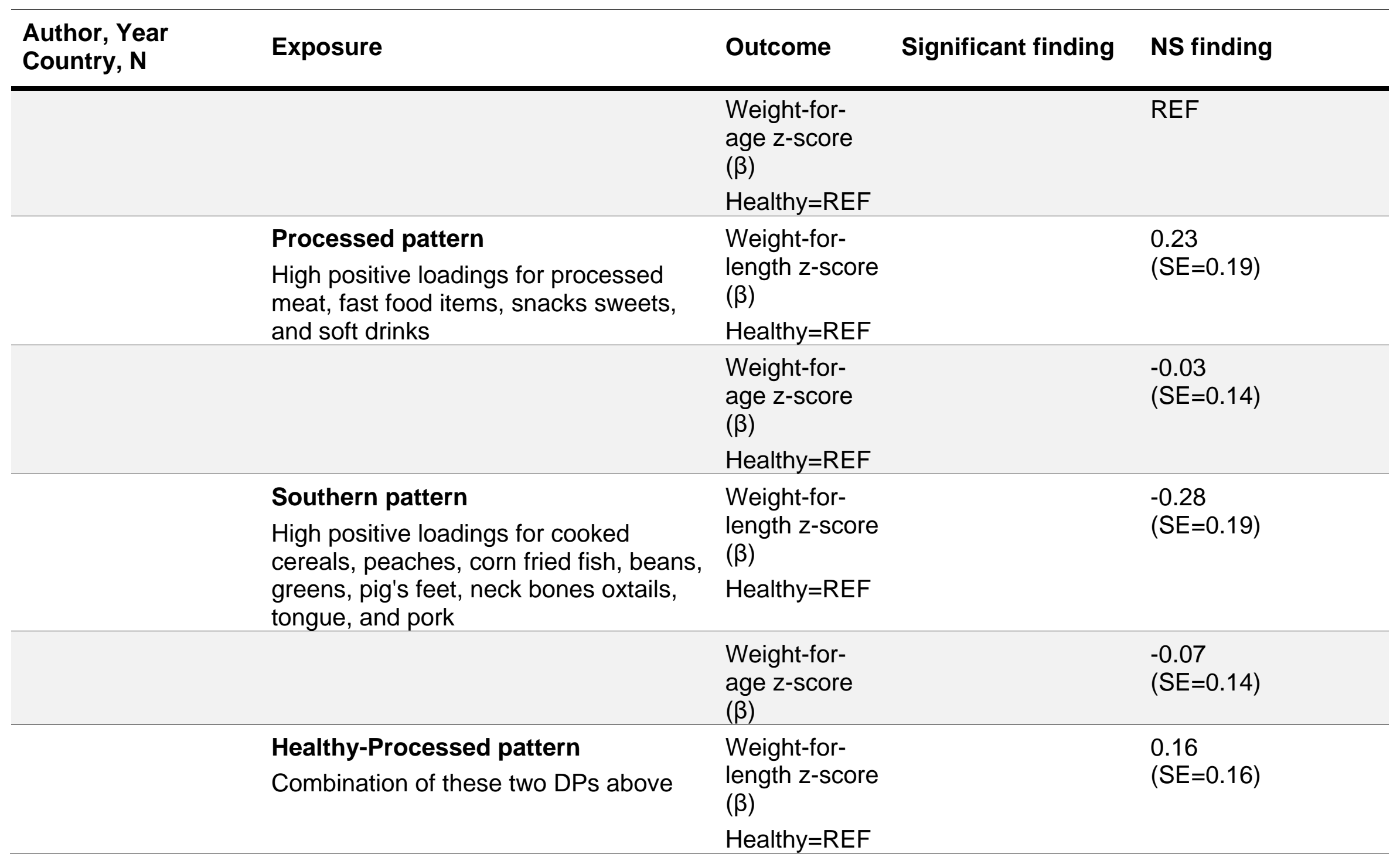




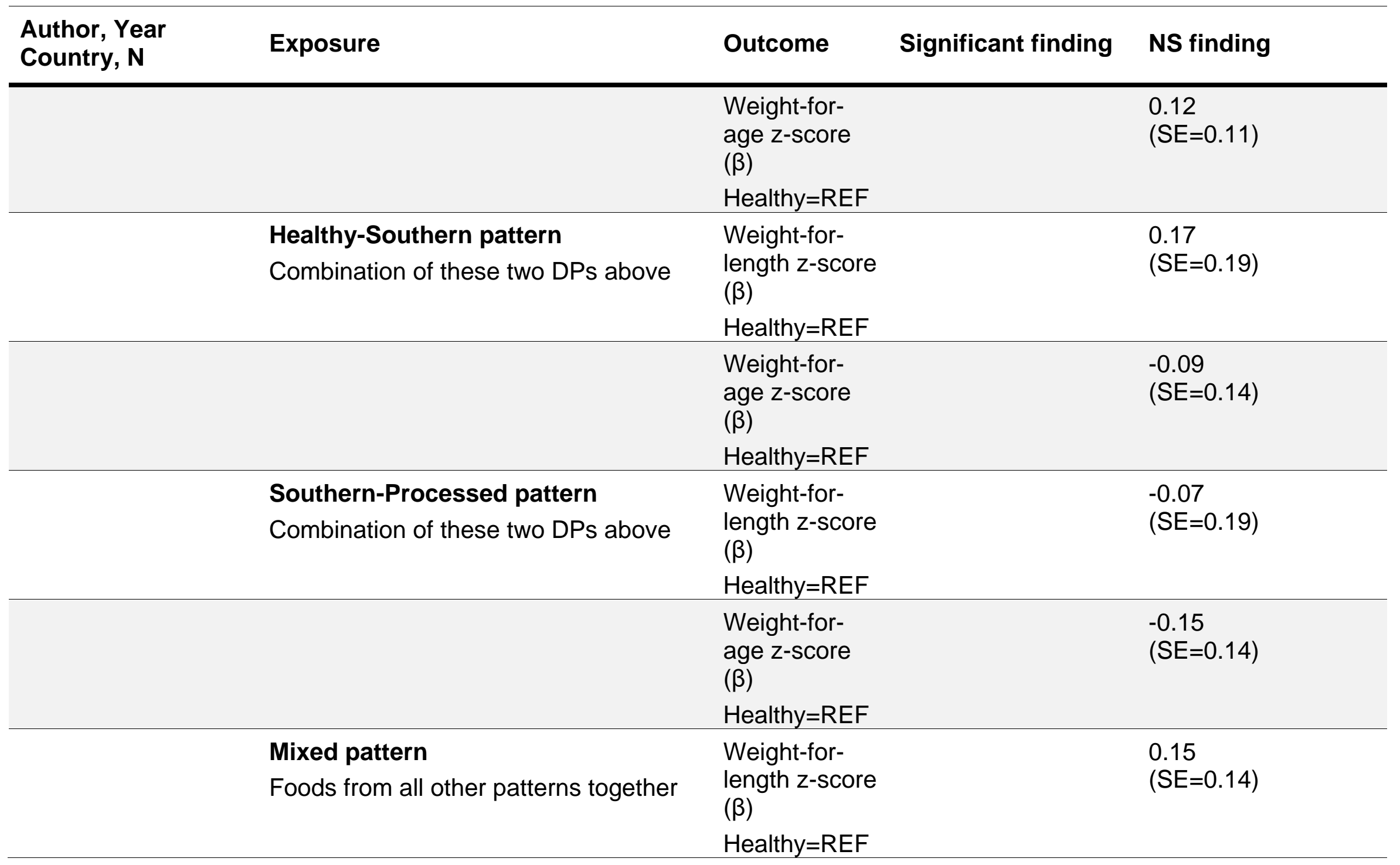




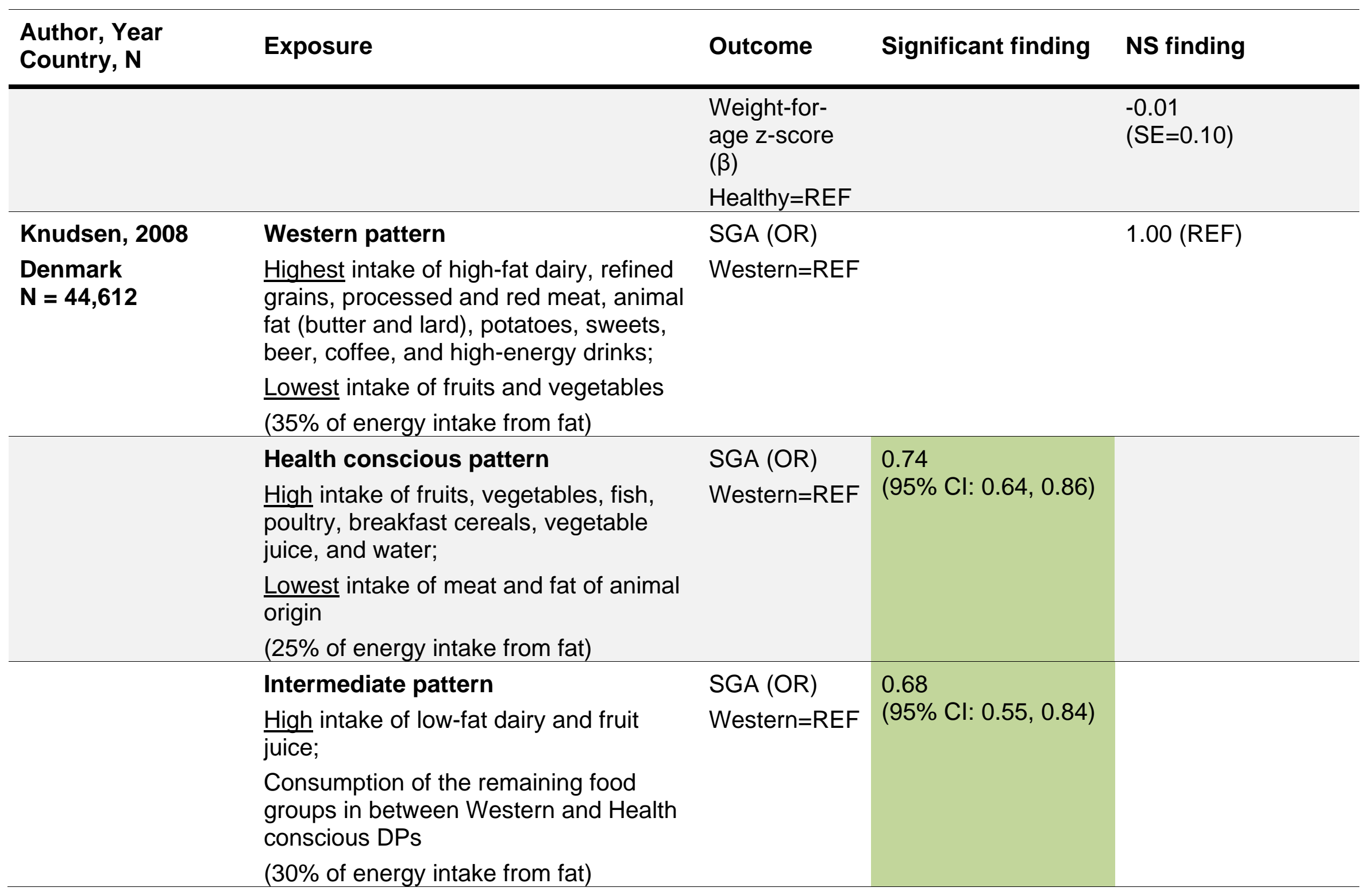




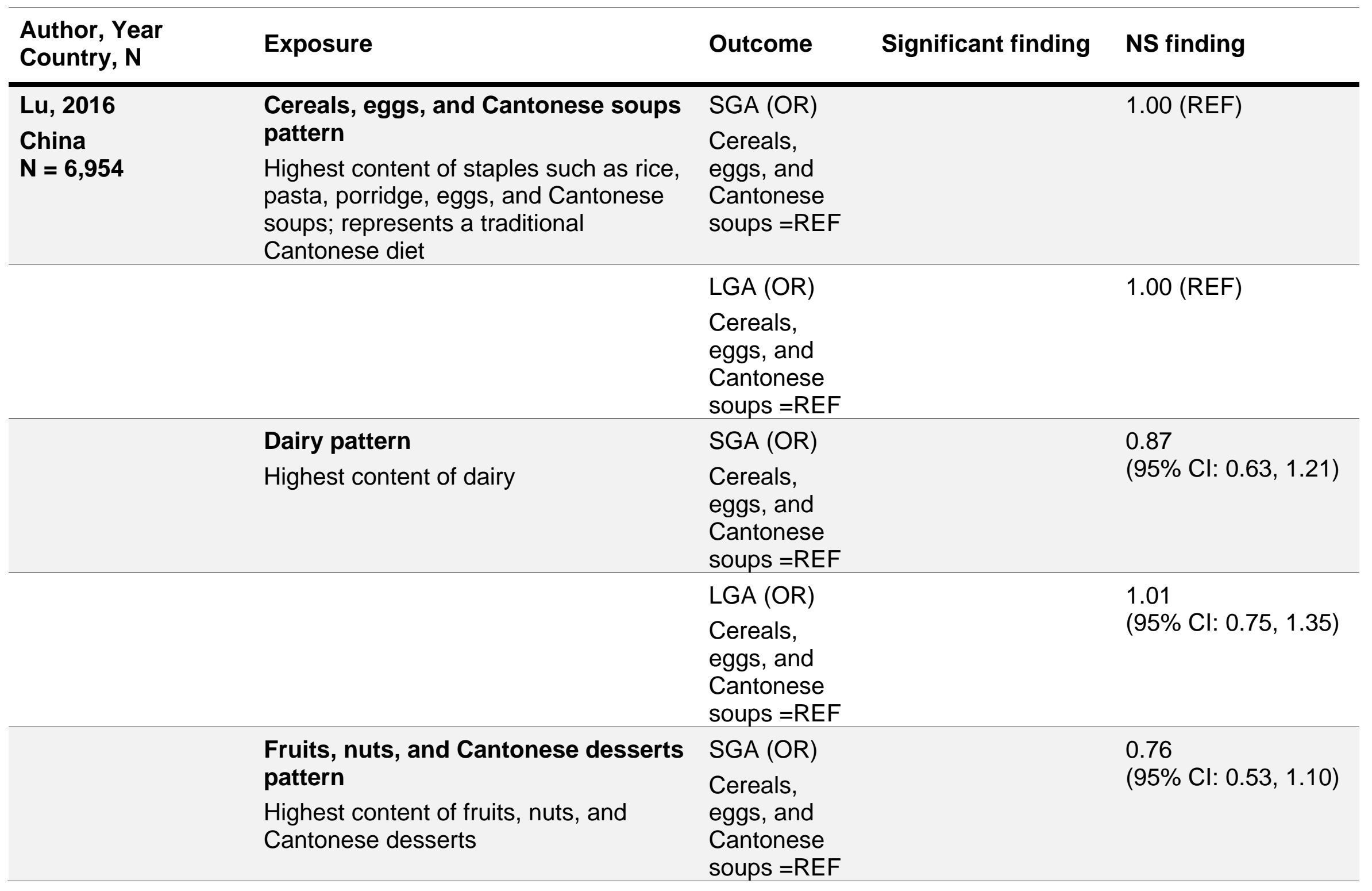




\begin{tabular}{|c|c|c|c|c|}
\hline $\begin{array}{l}\text { Author, Year } \\
\text { Country, N }\end{array}$ & Exposure & Outcome & Significant finding & NS finding \\
\hline & & $\begin{array}{l}\text { LGA (OR) } \\
\text { Cereals, } \\
\text { eggs, and } \\
\text { Cantonese } \\
\text { soups =REF }\end{array}$ & & $\begin{array}{l}1.14 \\
(95 \% \mathrm{Cl}: 0.84,1.54)\end{array}$ \\
\hline & $\begin{array}{l}\text { Meats pattern } \\
\text { Highest content of red meat and } \\
\text { processed meat }\end{array}$ & $\begin{array}{l}\text { SGA (OR) } \\
\text { Cereals, } \\
\text { eggs, and } \\
\text { Cantonese } \\
\text { soups =REF }\end{array}$ & & $\begin{array}{l}0.95 \\
(95 \% \mathrm{Cl}: 0.69,1.30)\end{array}$ \\
\hline & & $\begin{array}{l}\text { LGA (OR) } \\
\text { Cereals, } \\
\text { eggs, and } \\
\text { Cantonese } \\
\text { soups=REF }\end{array}$ & & $\begin{array}{l}0.75 \\
(95 \% \mathrm{Cl}: 0.56,1.02)\end{array}$ \\
\hline & $\begin{array}{l}\text { Vegetables pattern } \\
\text { Highest content of leafy and cruciferous } \\
\text { vegetables }\end{array}$ & $\begin{array}{l}\text { SGA (OR) } \\
\text { Cereals, } \\
\text { eggs, and } \\
\text { Cantonese } \\
\text { soups=REF }\end{array}$ & & $\begin{array}{l}0.77 \\
(95 \% \mathrm{Cl}: 0.56,1.05)\end{array}$ \\
\hline & & $\begin{array}{l}\text { LGA (OR) } \\
\text { Cereals, } \\
\text { eggs, and } \\
\text { Cantonese } \\
\text { soups=REF }\end{array}$ & & $\begin{array}{l}1.03 \\
(95 \% \mathrm{Cl}: 0.79,1.36)\end{array}$ \\
\hline
\end{tabular}




\begin{tabular}{|c|c|c|c|c|}
\hline $\begin{array}{l}\text { Author, Year } \\
\text { Country, N }\end{array}$ & Exposure & Outcome & Significant finding & NS finding \\
\hline & Varied pattern & SGA (OR) & & 0.77 \\
\hline & $\begin{array}{l}\text { Relatively high intakes of mixed food, } \\
\text { including noodles, bread, root } \\
\text { vegetables, melon vegetables, } \\
\text { mushrooms, sea vegetables, bean } \\
\text { vegetables, processed vegetables, } \\
\text { poultry, animal organ meat, fish, other } \\
\text { seafood, bean products, yoghourt, } \\
\text { sweet beverages, puffed food, } \\
\text { confectioneries, and snacks }\end{array}$ & $\begin{array}{l}\text { Cereals, } \\
\text { eggs, and } \\
\text { Cantonese } \\
\text { soups=REF }\end{array}$ & & (95\% Cl: $0.57,1.04)$ \\
\hline & & LGA (OR) & & 1.10 \\
\hline & & $\begin{array}{l}\text { Cereals, } \\
\text { eggs, and } \\
\text { Cantonese } \\
\text { soups=REF }\end{array}$ & & (95\% Cl: $0.85,1.42)$ \\
\hline \multirow{4}{*}{$\begin{array}{l}\text { Northstone, } 2008 \\
\text { England } \\
\mathrm{N}=12,053\end{array}$} & Health-conscious pattern & Birth weight & $39.87 \mathrm{~g}$ & \\
\hline & $\begin{array}{l}\text { High loadings on salad, fruit, rice, } \\
\text { pasta, breakfast cereals, fish, eggs, } \\
\text { pulses, fruit juices, poultry and non- } \\
\text { white bread }\end{array}$ & $\begin{array}{l}\text { ( } \beta \text { per } 1 \mathrm{SD} \\
\text { increase in } \\
\text { DP score) }\end{array}$ & $\begin{array}{l}(95 \% \mathrm{Cl}: 29.59 \\
50.16)\end{array}$ & \\
\hline & Traditional pattern & Birth weight & & $6.63 g$ \\
\hline & $\begin{array}{l}\text { High loadings on green vegetables and } \\
\text { root vegetables, potatoes, peas and to } \\
\text { some extent red meat and poultry }\end{array}$ & $\begin{array}{l}\text { ( } \beta \text { per } 1 \mathrm{SD} \\
\text { increase in } \\
\text { DP score) }\end{array}$ & & $\begin{array}{l}(95 \% \mathrm{Cl}:-3.40 \\
16.66)\end{array}$ \\
\hline
\end{tabular}




\begin{tabular}{|c|c|c|c|c|}
\hline $\begin{array}{l}\text { Author, Year } \\
\text { Country, N }\end{array}$ & Exposure & Outcome & Significant finding & NS finding \\
\hline & Processed pattern & Birth weight & $-18.03 g$ & \\
\hline & $\begin{array}{l}\text { High loadings on high-fat processed } \\
\text { foods, such as meat pies, sausages } \\
\text { and burgers, fried foods, pizza, chips } \\
\text { and crisps }\end{array}$ & $\begin{array}{l}\text { ( } \beta \text { per } 1 \text { SD } \\
\text { increase in } \\
\text { DP score) }\end{array}$ & $\begin{array}{l}\text { (95\% Cl: }-29.74,- \\
6.33)\end{array}$ & \\
\hline & Confectionery pattern & \multirow{2}{*}{\multicolumn{2}{|c|}{$\begin{array}{l}\text { Birth weight } \\
\text { ( } \beta \text { per } 1 \mathrm{SD} \\
\text { increase in } \\
\text { DP score) }\end{array}$}} & \multirow{2}{*}{$\begin{array}{l}-1.25 \mathrm{~g} \\
(95 \% \mathrm{Cl}:-12.90 \\
10.41)\end{array}$} \\
\hline & $\begin{array}{l}\text { High intakes of confectionery and other } \\
\text { foods with high sugar content such as } \\
\text { chocolate, sweets, biscuits, cakes and } \\
\text { other puddings }\end{array}$ & & & \\
\hline & Vegetarian pattern & \multirow{2}{*}{$\begin{array}{l}\text { Birth weight } \\
\text { ( } \beta \text { per } 1 \text { SD } \\
\text { increase in } \\
\text { DP score })\end{array}$} & \multirow{2}{*}{$\begin{array}{l}-15.47 \mathrm{~g} \\
(95 \% \mathrm{Cl}:-25.44,- \\
5.50)\end{array}$} & \\
\hline & $\begin{array}{l}\text { Loaded highly on meat substitutes, } \\
\text { pulses, nuts and herbal tea and high } \\
\text { negative loadings were seen with red } \\
\text { meat and poultry }\end{array}$ & & & \\
\hline \multirow{4}{*}{$\begin{array}{l}\text { Okubo, } 2012 \\
\text { Japan } \\
N=803\end{array}$} & Meat and eggs pattern & \multirow{2}{*}{$\begin{array}{l}\text { Birth weight } \\
\text { (mean) }\end{array}$} & & \multirow{2}{*}{$\begin{array}{l}3,105 \mathrm{~g} \\
(95 \% \mathrm{Cl}: 3,069 \\
3,141)\end{array}$} \\
\hline & $\begin{array}{l}\text { High intake of beef and pork, processed } \\
\text { meat, chicken, eggs, butter and dairy } \\
\text { products }\end{array}$ & & & \\
\hline & & \multirow{2}{*}{$\begin{array}{l}\text { SGA (OR) } \\
\text { Rice, fish, } \\
\text { and } \\
\text { vegetables= } \\
\text { REF }\end{array}$} & & \multirow{2}{*}{$\begin{array}{l}4.32 \\
(95 \% \mathrm{Cl}: 0.92,20.3)\end{array}$} \\
\hline & & & & \\
\hline
\end{tabular}




\begin{tabular}{|c|c|c|c|c|}
\hline $\begin{array}{l}\text { Author, Year } \\
\text { Country, N }\end{array}$ & Exposure & Outcome & Significant finding & NS finding \\
\hline & $\begin{array}{l}\text { Wheat products pattern } \\
\text { High intake of bread, confectioneries, } \\
\text { fruit and vegetable juice, and soft drinks }\end{array}$ & $\begin{array}{l}\text { Birth weight } \\
\text { (mean) }\end{array}$ & $\begin{array}{l}3,073 \mathrm{~g} \\
(95 \% \mathrm{Cl}: 3,063, \\
3,111)^{*} \\
\text { *significantly } \\
\text { different than rice, } \\
\text { fish, and vegetables } \\
\text { pattern }\end{array}$ & \\
\hline & & $\begin{array}{l}\text { SGA (OR) } \\
\text { Rice, fish, } \\
\text { and } \\
\text { vegetables= } \\
\text { REF }\end{array}$ & $\begin{array}{l}5.24 \\
(95 \% \mathrm{Cl}: 1.13,24.4)\end{array}$ & \\
\hline & & $\begin{array}{l}\text { SGA (OR) } \\
\text { Rice, fish, } \\
\text { and } \\
\text { vegetables= } \\
\text { REF }\end{array}$ & & 1.00 (REF) \\
\hline
\end{tabular}




\begin{tabular}{|c|c|c|c|c|}
\hline $\begin{array}{l}\text { Author, Year } \\
\text { Country, N }\end{array}$ & Exposure & Outcome & Significant finding & NS finding \\
\hline \multicolumn{5}{|c|}{$\begin{array}{l}\text { Before Pregnancy/ } \\
\text { Before and/or } \\
\text { During Pregnancy }\end{array}$} \\
\hline \multirow{6}{*}{$\begin{array}{l}\text { Xie, } 2015 \\
\text { USA } \\
N=572\end{array}$} & High-calorie sweet pattern & \multirow{2}{*}{\multicolumn{2}{|c|}{$\begin{array}{l}\text { Birth weight } \\
\text { ( } \beta \text { for } 1 \text {-unit } \\
\text { increase in } \\
\text { the scale) }\end{array}$}} & $0.02 \mathrm{~kg}(\mathrm{SE}=0.02)$ \\
\hline & $\begin{array}{l}\text { Foods such as doughnuts, ice cream, } \\
\text { chocolate candy, regular candy, and } \\
\text { cookies }\end{array}$ & & & \\
\hline & High-calorie nonsweet pattern & \multirow{2}{*}{\multicolumn{2}{|c|}{$\begin{array}{l}\text { Birth weight } \\
\text { ( } \beta \text { for } 1 \text {-unit } \\
\text { increase in } \\
\text { the scale) }\end{array}$}} & $-0.004 \mathrm{~kg}(\mathrm{SE}=0.01)$ \\
\hline & $\begin{array}{l}\text { Steak, fried chicken, fried fish, pizza, } \\
\text { hot dogs, sausage, cheese, whole milk, } \\
\text { etc. }\end{array}$ & & & \\
\hline & Low-calorie pattern & \multirow{2}{*}{\multicolumn{2}{|c|}{$\begin{array}{l}\text { Birth weight } \\
\text { ( } \beta \text { for } 1 \text {-unit } \\
\text { increase in } \\
\text { the scale) }\end{array}$}} & $0.03 \mathrm{~kg}(\mathrm{SE}=0.03)$ \\
\hline & $\begin{array}{l}\text { Foods such as low-fat and skim milk, } \\
\text { grilled chicken, grilled fish, apples, and } \\
\text { breakfast cereal }\end{array}$ & & & \\
\hline \multicolumn{5}{|l|}{ Pregnancy } \\
\hline \multirow{2}{*}{$\begin{array}{l}\text { Clapp, } 1997 \\
\text { USA } \\
N=12\end{array}$} & $\begin{array}{l}\text { Aboriginal Carbohydrate Diet } \\
\text { (Low-Gl) }\end{array}$ & \multirow{2}{*}{$\begin{array}{l}\text { Birth weight } \\
\text { (mean } \pm \\
\text { SEM) }\end{array}$} & \multirow{2}{*}{$\begin{array}{l}3.27 \pm 0.12 \mathrm{~kg}^{*} \\
\text { * significantly } \\
\text { different than } \\
\text { cafeteria } \\
\text { carbohydrate diet }\end{array}$} & \\
\hline & $\begin{array}{l}\text { Carbs from unprocessed whole grains, } \\
\text { fruits, beans, vegetables, and many } \\
\text { dairy products; includes most dense } \\
\text { whole grain and multigrain breads, bran } \\
\text { cereals, pastas, fresh fruits and } \\
\text { vegetables, yogurt, ice cream, and nuts }\end{array}$ & & & \\
\hline
\end{tabular}




\begin{tabular}{|c|c|c|c|c|}
\hline $\begin{array}{l}\text { Author, Year } \\
\text { Country, N }\end{array}$ & Exposure & Outcome & Significant finding & NS finding \\
\hline & $\begin{array}{l}\text { Cafeteria Carbohydrate Diet } \\
\text { (High-GI) } \\
\text { Carbs from highly processed grains, } \\
\text { root vegetables, and simple sugars; } \\
\text { includes many highly refined breads, } \\
\text { potatoes, instant rice, most breakfast } \\
\text { cereals, desserts, and snack-type foods }\end{array}$ & $\begin{array}{l}\text { Birth weight } \\
\text { (mean } \pm \\
\text { SEM) }\end{array}$ & $\begin{array}{l}4.25 \pm 0.11 \mathrm{~kg}^{*} \\
{ }^{*} \text { significantly } \\
\text { different than } \\
\text { aboriginal } \\
\text { carbohydrate diet }\end{array}$ & \\
\hline
\end{tabular}




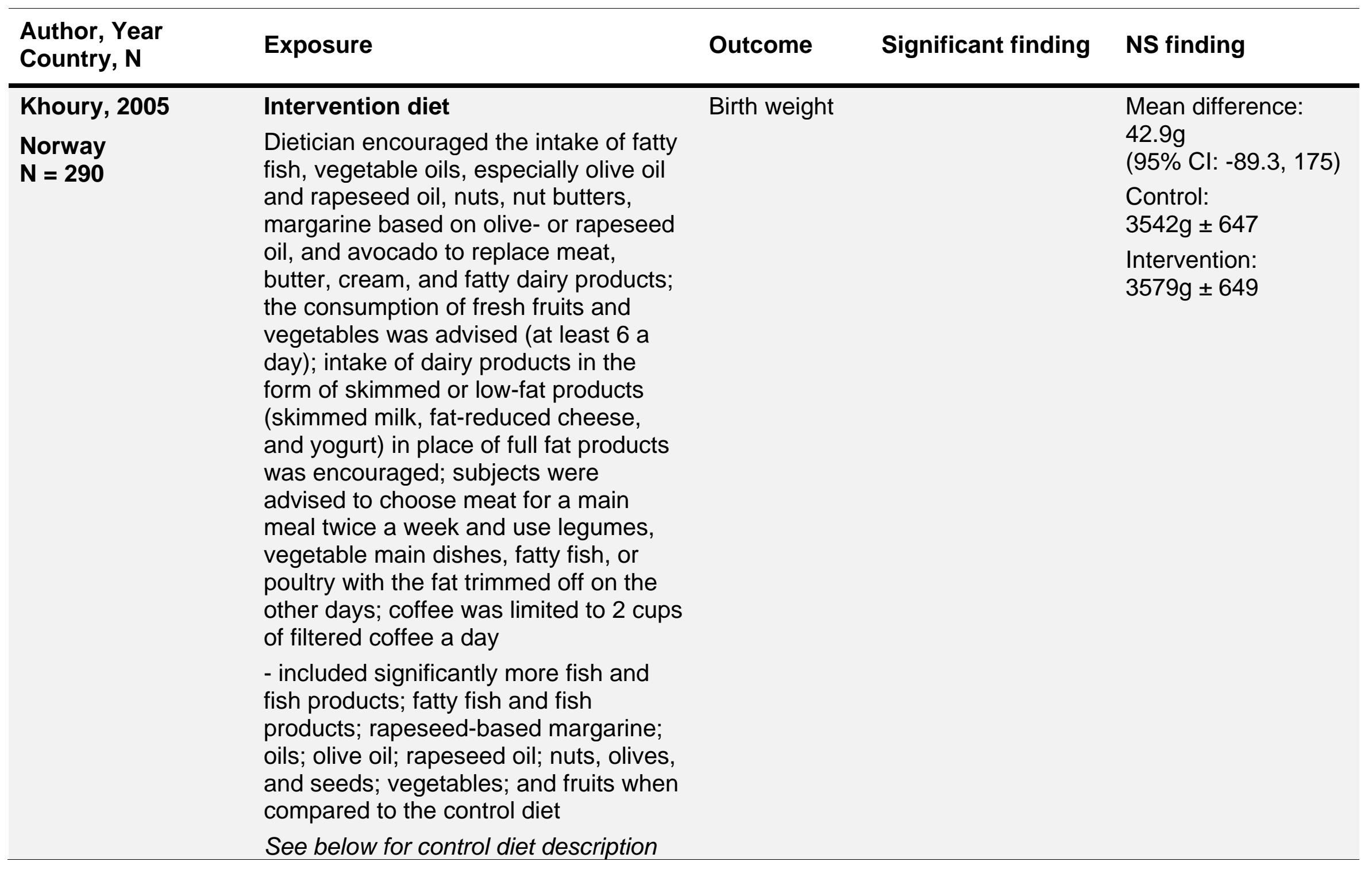




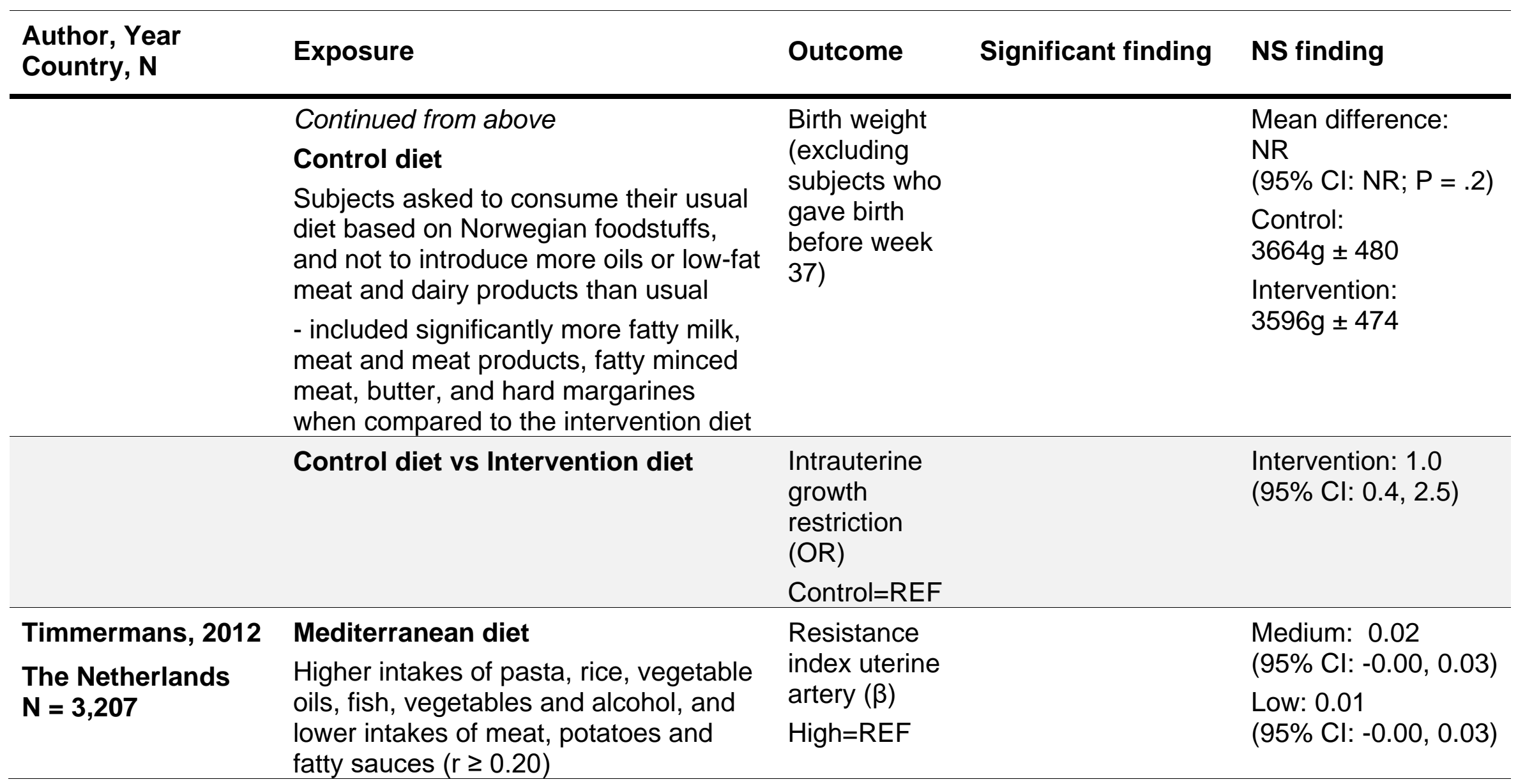




\begin{tabular}{|c|c|c|c|c|}
\hline $\begin{array}{l}\text { Author, Year } \\
\text { Country, N }\end{array}$ & Exposure & Outcome & Significant finding & NS finding \\
\hline & & \multirow{3}{*}{\multicolumn{2}{|c|}{$\begin{array}{l}\text { Head } \\
\text { circumference } \\
\text { standard } \\
\text { deviation } \\
\text { score }(\beta)\end{array}$}} & Mid-pregnancy: \\
\hline & & & & $\begin{array}{l}\text { Medium: } 0.01 \\
(95 \% \mathrm{Cl}:-0.08,0.10)\end{array}$ \\
\hline & & & & $\begin{array}{l}\text { Low: } 0.01 \\
(95 \% \mathrm{Cl}:-0.09,0.10)\end{array}$ \\
\hline & & \multirow{3}{*}{\multicolumn{2}{|c|}{ High=REF }} & $\begin{array}{l}\text { (95\% CI: }-0.09,0.10) \\
\text { Late-pregnancy: }\end{array}$ \\
\hline & & & & $\begin{array}{l}\text { Medium: }-0.03 \\
(95 \% \mathrm{Cl}:-0.12,0.05)\end{array}$ \\
\hline & & & & $\begin{array}{l}\text { Low: }-0.08 \\
(95 \% \mathrm{Cl}:-0.17,0.01)\end{array}$ \\
\hline & & \multirow{3}{*}{$\begin{array}{l}\text { Abdominal } \\
\text { circumference } \\
\text { standard } \\
\text { deviation } \\
\text { score }(\beta)\end{array}$} & \multirow{5}{*}{$\begin{array}{l}\text { Late-pregnancy: } \\
\text { Low: }-0.16 \\
(95 \% \text { Cl: }-0.25,- \\
0.07)\end{array}$} & Mid-pregnancy: \\
\hline & & & & $\begin{array}{l}\text { Medium: }-0.03 \\
(95 \% \mathrm{Cl}:-0.12,0.06)\end{array}$ \\
\hline & & & & Low: -0.05 \\
\hline & & \multirow[t]{2}{*}{ High=REF } & & Late-pregnancy: \\
\hline & & & & $\begin{array}{l}\text { Medium: }-0.06 \\
(95 \% \mathrm{Cl}:-0.15,0.03)\end{array}$ \\
\hline
\end{tabular}




\begin{tabular}{|c|c|c|c|c|}
\hline $\begin{array}{l}\text { Author, Year } \\
\text { Country, N }\end{array}$ & Exposure & Outcome & Significant finding & NS finding \\
\hline & & \multirow{2}{*}{\multicolumn{2}{|c|}{$\begin{array}{l}\text { Femur length } \\
\text { standard } \\
\text { deviation } \\
\text { score }(\beta)\end{array}$}} & Mid-pregnancy: \\
\hline & & & & $\begin{array}{l}\text { Medium: } 0.02 \\
(95 \% \text { Cl:-0.06, 0.11) }\end{array}$ \\
\hline & & \multicolumn{2}{|l|}{ High=REF } & $\begin{array}{l}\text { Low: } 0.07 \\
\text { (95\% Cl: }-0.02,0.16)\end{array}$ \\
\hline & & & & Late-pregnancy: \\
\hline & & & & $\begin{array}{l}\text { Medium: } 0.00 \\
(95 \% \text { Cl:-0.08, 0.08) }\end{array}$ \\
\hline & & & & $\begin{array}{l}\text { Low: } 0.01 \\
(95 \% \text { Cl: }-0.08,0.09)\end{array}$ \\
\hline & & \multirow{3}{*}{$\begin{array}{l}\text { Estimated } \\
\text { fetal weight } \\
\text { standard } \\
\text { deviation } \\
\text { score }(\beta)\end{array}$} & \multirow{5}{*}{$\begin{array}{l}\text { Mid-pregnancy: } \\
\text { Low: }-0.01 \\
(95 \% \mathrm{Cl}:-0.09,- \\
0.08) \\
\text { Late-pregnancy: } \\
\text { Low: }-0.11 \\
(95 \% \mathrm{Cl}:-0.20,- \\
0.02)\end{array}$} & Mid-pregnancy: \\
\hline & & & & $\begin{array}{l}\text { Medium: } 0.08 \\
(95 \% \text { Cl:-0.08, 0.09) }\end{array}$ \\
\hline & & & & Late-pregnancy: \\
\hline & & \multirow{2}{*}{ High=REF } & & Medium: -0.07 \\
\hline & & & & (95\% Cl:-0.16, 0.02) \\
\hline
\end{tabular}




\begin{tabular}{|c|c|c|c|c|}
\hline $\begin{array}{l}\text { Author, Year } \\
\text { Country, N }\end{array}$ & Exposure & Outcome & Significant finding & NS finding \\
\hline & & \multirow{4}{*}{$\begin{array}{l}\text { Birth weight } \\
(\beta) \\
\text { High=REF }\end{array}$} & $\begin{array}{l}\text { Medium: }-58.0 \mathrm{~g} \\
(95 \% \mathrm{Cl}:-95.8,-\end{array}$ & \\
\hline & & & $\begin{array}{l}20.3) \\
\text { Difference in SDS: } \\
-0.16 \\
(95 \% \mathrm{Cl}:-0.24,- \\
0.07)\end{array}$ & \\
\hline & & & $\begin{array}{l}\text { Low: }-72.0 \mathrm{~g} \\
\text { (95\% Cl: }-110.8,- \\
33.3)\end{array}$ & \\
\hline & & & $\begin{array}{l}\text { Difference in SDS: } \\
-0.21 \\
(95 \% \mathrm{Cl}:-0.30,- \\
0.12)\end{array}$ & \\
\hline
\end{tabular}




\section{Assessment of the body of evidence}

A grade was not assignable for this body of evidence. The individual grading elements are discussed below.

\section{Internal validity (determined with the NEL Bias Assessment Tool):}

- Study Design: The data were primarily observational in nature, making it difficult to determine causal effect of the dietary patterns. Further, the characteristics of the study participants were also heterogeneous.

- Exposure:

○ Data were primarily self-reported, thus possibly affecting the validity of the data collected.

- Studies used a variety of tools to measure participants' dietary intake:

- Food frequency questionnaires (FFQ) were the primary measurement tool. A few of the FFQ used were neither validated $(15,17,18)$ nor assessed for reliability, and the type of FFQ used varied between studies.

- Some studies used other methods, including diet history questionnaires $(6,10), 24-\mathrm{hr}$ recall $(10)$, a 3-day food diary (11) and dietary recall (17).

- When the subjects were randomized to a particular diet, compliance was assessed periodically $(1,21)$.

- Furthermore, the methods used to create dietary patterns were heterogeneous.

- Timing:

- Variation in the timing of exposure assessment and the duration of recall periods across studies makes it difficult to draw conclusions for the entire periods before and during pregnancy.

- Grieger et al. exclusively assessed maternal diet before pregnancy.

- Monteagudo et al., Gresham et al. and Xie et al. measured diet during a wide window spanning from before pregnancy to the end of pregnancy, and they did not delineate their findings based on timing (before pregnancy vs. during pregnancy).

- Rodriguez-Bernal et al., Bouwland-Both et al. and Timmermans et al. assessed diet during the first trimester, with the recall period spanning from conception until the point of measurement.

- Hillesund et al., Chia et al., Colon-Ramos et al., Knudsen et al., and Lu et al. all measured maternal diet during the second trimester (with variable time periods of recall).

- Northstone et al. measured diet during the third trimester and captured current consumption.

- Several studies assessed diet across trimesters, which included varied recall periods.

- Confounders:

- Key confounders (parity, educational attainment, smoking status, race/ethnicity, maternal age, family poverty income ratio, pre-pregnancy $\mathrm{BMI}$, mean total energy intake, and hypertensive disorders of pregnancy) were not consistently accounted for across studies. This limits the internal validity of study findings. 
- Six studies did not adjust for pre-pregnancy BMI, two of which were RCTs. Khoury et al. noted that pre-pregnancy BMI did not differ between intervention and control diet groups, whereas Clapp did not compare pre-pregnancy BMl across groups.

- Furthermore, only six of 21 studies adjusted for maternal energy intake, a key confounder in the association between dietary patterns and birth weight outcomes.

- None of the studies included in this review assessed effect measure modification between dietary patterns and maternal pre-pregnancy BMI in the context of birth weight outcomes. Failing to examine these potential interactions could have prevented these studies from observing associations.

- Outcome:

- Outcomes were assessed in a variety of ways across studies included in this review.

- Just one-third of studies $(n=7)$ used both gestational age- and sexspecific cut-off values when defining SGA, AGA, LGA and IUGR $(1,5,6,11,13,14,16)$.

- Twelve studies reported birth weight adjusted for gestational age as an outcome $(1,5-9,11-14,16,20)$.

- Nine studies reported birth weight, alone, without standardizing for gestational age or sex or using z-scores $(2-4,10,15,17-19,21)$.

- Four studies included subjects born at a specified gestational age:

- Hillesund ( $\geq 37$ to $\leq 42$ weeks)

- Poon ( $\geq 35$ to $\leq 43.5$ weeks)

- Shapiro ( $\geq 32$ weeks)

- Okubo ( $\geq 37$ to $\leq 41$ weeks)

- The use of birth weight values unadjusted for infant sex or gestational age at delivery in many of these studies makes these data difficult to interpret.

\section{Consistency:}

- There was heterogeneity in study findings in this body of evidence. Some studies found an association between dietary patterns and birth weight outcomes $(n=11)$, while others did not $(n=10)$. This varied by the specific measure of size at birth that was used in each study.

- Small- or large-for-gestational age:

- Five of the 12 studies showed an association between dietary patterns and SGA $(5,7,8,13,16)$.

- Two of the six studies noted that a greater adherence to a dietary pattern (specifically, the New Nordic Diet and a vegetable, fruit and white rice pattern) was associated with an increased risk of $\operatorname{LGA}(5,11)$. Monteagudo et al. observed that a higher Mediterranean diet score was predictive of newborn overweight $(>3,500 \mathrm{~g})$ when compared to newborn underweight $(<2,500 \mathrm{~g})$; however, the birth weight was not standardized for gestational age or z-score.

- Monteagudo et al. findings may be explained in part by their structuring of the outcome as the likelihood of overweight versus underweight as the reference group. This likely contributed to their 
large observed effect size.

- Birth weight:

- Birth weight standardized for gestational age was reported in three studies $(8,9,12)$, of which only Rodriguez-Bernal et al. reported a positive association with the birth weight.

- Crude birth weight, without standardizing for gestational age or sex (i.e, using percentiles or z-scores), was reported in 9 studies. Of these, four showed that adherence to a healthy dietary pattern was associated with birth weight $(2,7,15,21)$. Among studies that reported low birth weight as an outcome $(n=3)$, none showed an association with maternal dietary patterns $(4,18,20)$.

- The mean birth weight for included studies was above $3,000 \mathrm{~g}$ (range: $3,103 \mathrm{~g}(16)$ to $\sim 3,760 \mathrm{~g} \mathrm{(21))}$, indicating that babies were born at a healthy weight, overall. This may explain why many studies did not detect an association between healthier dietary patterns and increased birth weight.

- Dietary patterns were associated with other outcomes such as crown-rump length (3), estimated fetal weight during mid- and late-pregnancy and latepregnancy abdominal circumference (2), but there was no association with other fetal measures (head circumference, femur length) or weight for length zscore.

Impact:

- Most of the studies in the body of evidence directly examined the relationship between different dietary patterns or different levels of adherence to a dietary pattern and birth weight. However, there were a few exceptions. First, the RCT by Khoury et al. assessed IUGR and birth weight as secondary outcomes. The primary study was focused on understanding the relationship between a cholesterol-lowering diet during pregnancy and maternal, cord, and neonatal cholesterol levels (1). Second, Shapiro et al. was designed to assess the association between maternal diet and neonatal adiposity, with birth weight as a secondary outcome.

- The lack of consistency across studies limits the practical/clinical significance of this review.

\section{Adequacy:}

- A substantial number of studies were included in this review. The evidence base included 21 studies comprising 2 RCTs, 18 prospective cohort studies, and 1 retrospective cohort study.

- Most of the studies were conducted by independent research groups. The only exception was Timmermans et al. and Bouwland-Both et al., who both used data from the Generation R study and also had overlapping authorship.

- Sample sizes varied from 12 subjects to 66,597 subjects. The median sample size was 1,079 . None of the studies reported power calculations. A few studies $(1,17,19-21)$ had lower sample sizes, which might have limited their ability to detect significant differences had such differences been present.

\section{Generalizability:}

- There are serious limitations to the generalizability of these findings. 
- Only seven out of 21 studies were conducted in the U.S.

- Adolescent, minority, and lower-SES populations are underrepresented

in the body of evidence. Only one study was conducted primarily in adolescent girls (17).

- It is unknown if the findings would apply to more diverse samples before and during pregnancy.

\section{Other limitations/considerations:}

Many of the studies did not use a standardized outcome measure (such as SGA, LGA, or gestational age- and sex-specific birth weight). Even among those that used a standardized measure, there was heterogeneity in how it was defined. Some studies used observed distributions of fetal size that are standardized for gestational age and sex in a defined population. Meanwhile, others used observed distributions that were customized based on maternal characteristics (e.g. maternal parity, height, ethnicity) or standards derived from a healthy population selected to reflect optimal growth ${ }^{37}$.

As discussed above, none of the studies assessed effect measure modification between dietary patterns and maternal pre-pregnancy BMI in the context of birth weight outcomes and only a limited number of studies $(n=6)$ adjusted for mean total energy intake. These limitations could have possibly influenced the study findings.

Many different methods (sometimes using the same nomenclature) were used to define and assess dietary patterns, making it difficult to compare or contrast results across studies. Journal editors and peer-reviewers may be less willing to publish studies that replicate others' findings, which could have resulted in an evidence base with a wide array of dietary patterns. It is important for the editors and peer-reviewers to understand the need for publishing studies that replicate dietary patterns, in addition to publishing studies that assess unique dietary patterns. ${ }^{38}$

\section{Research recommendations}

To assess the relationship between dietary patterns before and during pregnancy and birth weight outcomes more adequately, additional research is needed that should:

- Include diverse populations from the U.S. and elsewhere with varying age groups (including adolescents) and different racial/ethnic and socioeconomic backgrounds.

- Assess effect measure modification by pre-pregnancy BMI and gestational weight gain.

- Use a standardized birth size measure (such as one developed by the INTERGROWTH-21 $1^{\text {st }}$ project) that would enable valid comparisons between and within countries ${ }^{39}$.

- Include well-designed and sufficiently powered RCTs.

- Foster collaborative efforts across different regions and populations so that

\footnotetext{
37 Westerway, S. C., Papageorghiou, A. T., Hirst, J., Costa, F. D., Hyett, J., \& Walker, S. P. (2015). INTERGROWTH-21st - Time to standardise fetal measurement in Australia. Australas J Ultrasound Med, 18(3), 9195. doi:10.1002/j.2205-0140.2015.tb00206.x

38 U.S. Department of Health and Human Services and U.S. Department of Agriculture. (2015). 2015 - 2020 Dietary Guidelines for Americans. 8th Edition. Retrieved from https://health.gov/dietaryguidelines/2015/guidelines/.

39 Westerway, S. C., Papageorghiou, A. T., Hirst, J., Costa, F. D., Hyett, J., \& Walker, S. P. (2015).

INTERGROWTH-21st - Time to standardise fetal measurement in Australia. Australas J Ultrasound Med, 18(3), 9195. doi:10.1002/j.2205-0140.2015.tb00206.x
} 
dietary patterns can be more consistently scored, compared and reproduced across studies.

- Develop and validate novel epidemiological tools that can accurately capture the complexity of dietary habits.

- Promote harmonization of research methods across various cohorts and randomized trials, similar to the National Cancer Institute's Dietary Patterns Methods Project ${ }^{40}$.

- Adjust for key confounding factors in observational studies, including parity, educational attainment, smoking status, race/ethnicity, maternal age, family poverty income ratio, pre-pregnancy BMI, mean total energy intake and gestational weight gain.

40 Liese, A. D., Krebs-Smith, S. M., Subar, A. F., George, S. M., Harmon, B. E., Neuhouser, M. L., . . Reedy, J. (2015). The Dietary Patterns Methods Project: synthesis of findings across cohorts and relevance to dietary guidance. J Nutr, 145(3), 393-402. doi:10.3945/jn.114.205336 


\section{Included articles}

1. Khoury J, Henriksen T, Christophersen B, Tonstad S. Effect of a cholesterollowering diet on maternal, cord, and neonatal lipids, and pregnancy outcome: a randomized clinical trial. Am J Obstet Gynecol 2005;193(4):1292-301.

2. Timmermans $S$, Steegers-Theunissen RP, Vujkovic M, den Breeijen $\mathrm{H}$, Russcher $\mathrm{H}$, Lindemans J, Mackenbach J, Hofman A, Lesaffre EE, Jaddoe VV, et al. The Mediterranean diet and fetal size parameters: the Generation R Study. Br J Nutr 2012;108(8):1399-409.

3. Bouwland-Both MI, Steegers-Theunissen RP, Vujkovic M, Lesaffre EM, MookKanamori DO, Hofman A, Lindemans J, Russcher H, Jaddoe VW, Steegers EA. A periconceptional energy-rich dietary pattern is associated with early fetal growth: the Generation R study. BJOG 2013;120(4):435-45.

4. Gresham E, Collins CE, Mishra GD, Byles JE, Hure AJ. Diet quality before or during pregnancy and the relationship with pregnancy and birth outcomes: the Australian Longitudinal Study on Women's Health. Public Health Nutr 2016;19(16):2975-83.

5. Hillesund ER, Bere E, Haugen M, Overby NC. Development of a New Nordic Diet score and its association with gestational weight gain and fetal growth - a study performed in the Norwegian Mother and Child Cohort Study (MoBa). Public Health Nutr 2014;17(9):1909-18.

6. Poon AK, Yeung E, Boghossian N, Albert PS, Zhang C. Maternal Dietary Patterns during Third Trimester in Association with Birthweight Characteristics and Early Infant Growth. Scientifica (Cairo) 2013;2013:786409.

7. Chatzi L, Mendez M, Garcia R, Roumeliotaki T, Ibarluzea J, Tardon A, Amiano P, Lertxundi A, Iniguez C, Vioque J, et al. Mediterranean diet adherence during pregnancy and fetal growth: INMA (Spain) and RHEA (Greece) mother-child cohort studies. Br J Nutr 2012;107(1):135-45.

8. Rodriguez-Bernal CL, Rebagliato M, Iniguez C, Vioque J, Navarrete-Munoz EM, Murcia M, Bolumar F, Marco A, Ballester F. Diet quality in early pregnancy and its effects on fetal growth outcomes: the Infancia y Medio Ambiente (Childhood and Environment) Mother and Child Cohort Study in Spain. Am J Clin Nutr 2010;91(6):1659-66.

9. Rifas-Shiman SL, Rich-Edwards JW, Kleinman KP, Oken E, Gillman MW. Dietary quality during pregnancy varies by maternal characteristics in Project Viva: a US cohort. J Am Diet Assoc 2009;109(6):1004-11.

10. Shapiro AL, Kaar JL, Crume TL, Starling AP, Siega-Riz AM, Ringham BM, Glueck $\mathrm{DH}$, Norris JM, Barbour LA, Friedman JE, et al. Maternal diet quality in pregnancy and neonatal adiposity: the Healthy Start Study. Int J Obes (Lond) 2016;40(7):105662.

11. Chia AR, de Seymour JV, Colega M, Chen LW, Chan YH, Aris IM, Tint MT, Quah PL, Godfrey KM, Yap F, et al. A vegetable, fruit, and white rice dietary pattern during pregnancy is associated with a lower risk of preterm birth and larger birth size in a multiethnic Asian cohort: the Growing Up in Singapore Towards healthy Outcomes (GUSTO) cohort study. Am J Clin Nutr 2016;104(5):1416-23.

12. Colon-Ramos U, Racette SB, Ganiban J, Nguyen TG, Kocak M, Carroll KN, Volgyi E, Tylavsky FA. Association between dietary patterns during pregnancy and birth size measures in a diverse population in Southern US. Nutrients 2015;7(2):1318-32.

13. Knudsen VK, Orozova-Bekkevold IM, Mikkelsen TB, Wolff S, Olsen SF. Major dietary patterns in pregnancy and fetal growth. Eur J Clin Nutr 2008;62(4):463-70. 
14. Lu MS, Chen QZ, He JR, Wei XL, Lu JH, Li SH, Wen XX, Chan FF, Chen NN, Qiu $\mathrm{L}$, et al. Maternal Dietary Patterns and Fetal Growth: A Large Prospective Cohort Study in China. Nutrients 2016;8(5).

15. Northstone K, Ness AR, Emmett PM, Rogers IS. Adjusting for energy intake in dietary pattern investigations using principal components analysis. Eur J Clin Nutr 2008;62(7):931-8.

16. Okubo H, Miyake Y, Sasaki S, Tanaka K, Murakami K, Hirota Y, Osaka M, Child Health Study G, Kanzaki H, Kitada M, et al. Maternal dietary patterns in pregnancy and fetal growth in Japan: the Osaka Maternal and Child Health Study. Br J Nutr 2012;107(10):1526-33.

17. Xie Y, Madkour AS, Harville EW. Preconception Nutrition, Physical Activity, and Birth Outcomes in Adolescent Girls. J Pediatr Adolesc Gynecol 2015;28(6):471-6.

18. Kennedy ET. A prenatal screening system for use in a community-based setting. $J$ Am Diet Assoc 1986;86(10):1372-5.

19. Monteagudo C, Mariscal-Arcas M, Heras-Gonzalez L, Ibanez-Peinado D, Rivas A, Olea-Serrano F. Effects of maternal diet and environmental exposure to organochlorine pesticides on newborn weight in Southern Spain. Chemosphere 2016;156:135-42.

20. Grieger JA, Grzeskowiak LE, Clifton VL. Preconception dietary patterns in human pregnancies are associated with preterm delivery. J Nutr 2014;144(7):1075-80.

21. Clapp JF. Diet, exercise, and feto-placental growth. Archives of Gynecology and Obstetrics 1997;260(1-4):101-8. 


\section{ERROR! REFERENCE SOURCE NOT FOUND.}

The analytic framework (Figure 1) illustrates the overall scope of the project, including the population, the interventions and/or exposures, comparators, and outcomes of interest. It also includes definitions of key terms and identifies key confounders considered in the systematic review. This is the analytic framework for the systematic review conducted to examine the relationship between dietary patterns before and during pregnancy and gestational age- and sex-specific birth weight.

\section{Figure 1: Analytic framework}

Analytic Framework: What is the relationship between dietary patterns before and during pregnancy and gestational age- and sex-specific birth weight?

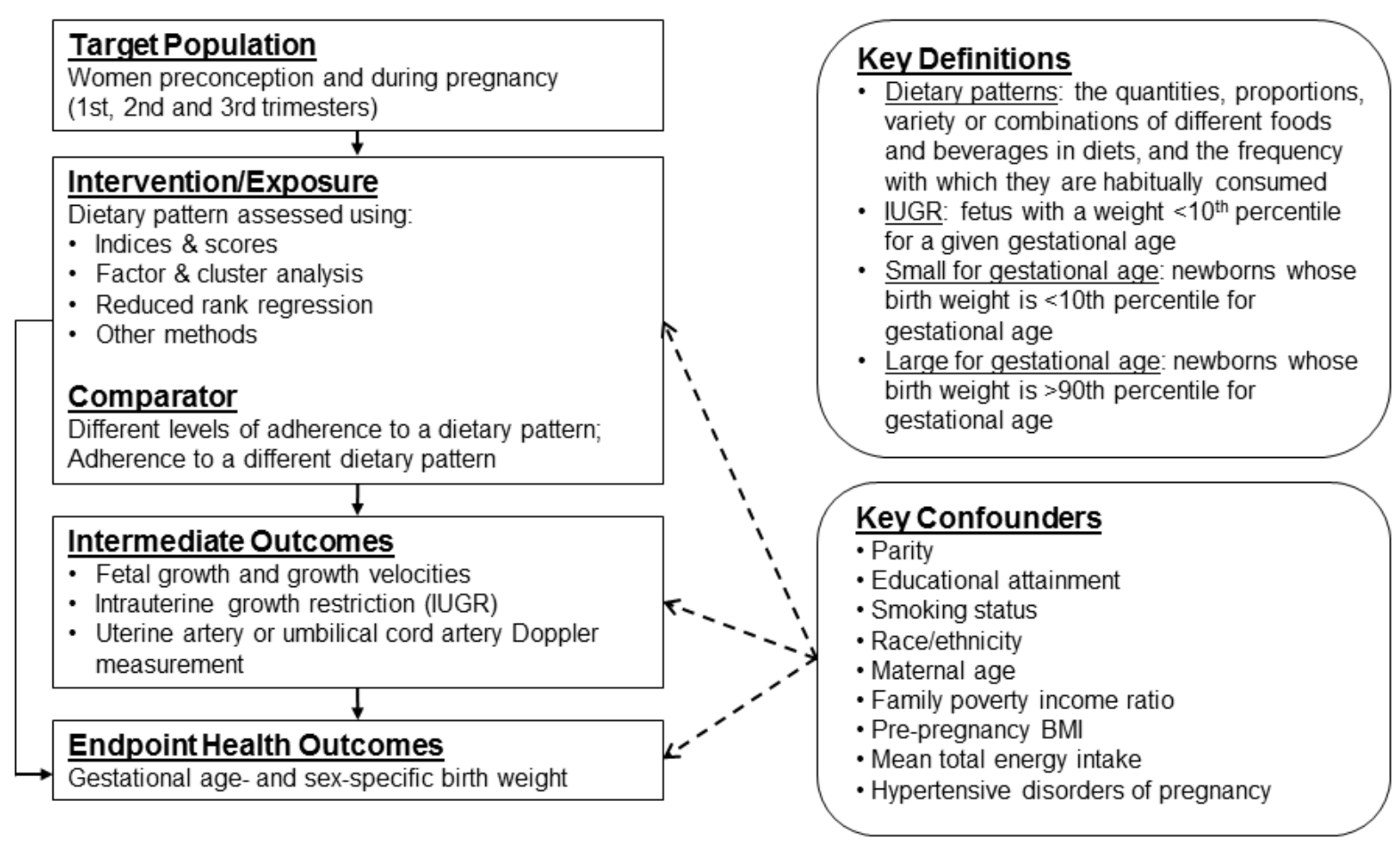




\section{Inclusion and exclusion criteria}

This table provides the inclusion and exclusion criteria for the systematic review question: what is the relationship between dietary patterns before and during pregnancy and gestational age- and sex-adjusted birth weight. The inclusion and exclusion criteria are a set of characteristics to determine which studies will be included or excluded in the systematic review.

Table 7. Inclusion and exclusion criteria

\begin{tabular}{|c|c|c|}
\hline Category & Inclusion Criteria & Exclusion Criteria \\
\hline $\begin{array}{l}\text { Study } \\
\text { Design }\end{array}$ & $\begin{array}{l}\text { - Randomized controlled trials } \\
\text { - Prospective cohort studies } \\
\text { - Retrospective cohort studies } \\
\text { - }\end{array}$ & $\begin{array}{l}\text { - Non-randomized controlled } \\
\text { - } \text { trials } \\
\text { - Cross-sectional studies } \\
\text { - Uncontrolled studies } \\
\text { - Pre/post studies with a control } \\
\text { - Pre/post studies without a } \\
\text { - } \text { - Narratrol } \\
\text { - Systematic reviews } \\
\text { - Meta-analyses }\end{array}$ \\
\hline $\begin{array}{l}\text { Exposure/ } \\
\text { Intervention }\end{array}$ & $\begin{array}{l}\text { - Studies that provide a description } \\
\text { of the dietary pattern(s) (i.e., foods } \\
\text { and beverages) consumed by } \\
\text { subjects and that methodologically } \\
\text { use: } \\
\circ \text { Indices \& scores } \\
\circ \quad \text { Cluster or factor analysis } \\
\circ \text { Reduced rank regression } \\
\circ \text { Other methods }\end{array}$ & $\begin{array}{l}\text { - Studies that do not provide a } \\
\text { description of the dietary } \\
\text { pattern(s) (i.e., foods and } \\
\text { beverages) consumed by } \\
\text { subjects }^{41}\end{array}$ \\
\hline Comparator & $\begin{array}{l}\text { - Different levels of adherence to a } \\
\text { dietary pattern } \\
\text { - Adherence to a different dietary } \\
\text { pattern }\end{array}$ & \\
\hline
\end{tabular}

${ }^{41}$ For example, a study would be excluded from the systematic review if the dietary pattern were labeled "vegetarian" but lacked a description of what foods/beverages were consumed as part of that dietary pattern. 


\begin{tabular}{|c|c|c|}
\hline Category & Inclusion Criteria & Exclusion Criteria \\
\hline Date Range & $\begin{array}{l}\text { - Studies published in the following } \\
\text { date range: } 1980 \text {-present (search } \\
\text { date) }\end{array}$ & \\
\hline Language & - Studies published in English & $\begin{array}{l}\text { - Studies published in } \\
\text { languages other than English }\end{array}$ \\
\hline \multirow[t]{2}{*}{$\begin{array}{l}\text { Study } \\
\text { Setting }\end{array}$} & $\begin{array}{l}\text { - Studies conducted in Very High } \\
\text { and High Human Development } \\
\text { Countries* }\end{array}$ & $\begin{array}{l}\text { - Studies conducted in Medium } \\
\text { and Low Human } \\
\text { Development Countries }{ }^{*}\end{array}$ \\
\hline & $\begin{array}{l}{ }^{*} \text { Determined using the most recent } \\
\text { Human Development Index }\end{array}$ & $\begin{array}{l}{ }^{*} \text { Determined using the most } \\
\text { recent Human Development } \\
\text { Index }\end{array}$ \\
\hline $\begin{array}{l}\text { Study } \\
\text { Duration }\end{array}$ & - Studies regardless of length & $\bullet$ \\
\hline Temporality & $\begin{array}{l}\text { - Studies when the exposure was } \\
\text { assessed prior to the outcome }\end{array}$ & $\begin{array}{l}\text { - Studies when the outcome } \\
\text { was assessed prior to the } \\
\text { exposure }\end{array}$ \\
\hline $\begin{array}{l}\text { Publication } \\
\text { Status }\end{array}$ & $\begin{array}{l}\text { - Studies published in peer- } \\
\text { reviewed journals }\end{array}$ & $\begin{array}{l}\text { Grey literature, including } \\
\text { unpublished data, } \\
\text { manuscripts, reports, } \\
\text { abstracts, conference } \\
\text { proceedings }\end{array}$ \\
\hline $\begin{array}{l}\text { Study } \\
\text { Subjects }\end{array}$ & $\begin{array}{l}\text { - Human subjects } \\
\text { - Adolescent girls and women } \\
\text { capable of becoming pregnant } \\
\text { (15-44 years) } \\
\text { - Pregnant girls and women (15-44 } \\
\text { years) - single and multiple } \\
\text { pregnancies } \\
\text { - Neonates }\end{array}$ & $\begin{array}{l}\text { - Animal and in vitro models } \\
\text { - Hospitalized patients, when } \\
\text { hospitalization is not related } \\
\text { to pregnancy, birth and } \\
\text { immediate postpartum } \\
\text { - Pregnancies conceived ONLY } \\
\text { using Assisted Reproductive } \\
\text { Technologies }\end{array}$ \\
\hline $\begin{array}{l}\text { Size of } \\
\text { Study } \\
\text { Groups }\end{array}$ & - Studies regardless of group size & $\bullet$ \\
\hline
\end{tabular}




\begin{tabular}{|c|c|c|}
\hline Category & Inclusion Criteria & Exclusion Criteria \\
\hline $\begin{array}{l}\text { Health } \\
\text { Status of } \\
\text { Study } \\
\text { Subjects }\end{array}$ & $\begin{array}{l}\text { - Studies conducted in generally } \\
\text { healthy women of reproductive } \\
\text { age, including women in pre/peri- } \\
\text { conception and pregnancy } \\
\text { - Studies conducted in samples with } \\
\text { elevated chronic disease risk or } \\
\text { pregnancy related conditions, or } \\
\text { that enroll some subjects with a } \\
\text { disease or with health outcome of } \\
\text { interest such as } \\
\circ \text { Anemia } \\
\circ \text { Gestational diabetes } \\
\circ \text { Hypertension } \\
\circ \text { Preeclampsia } \\
\circ \text { Hypermesis Gravidarum } \\
\circ \text { Previous adverse outcome } \\
\text { (e.g., preterm) } \\
\circ \text { Obesity }\end{array}$ & $\begin{array}{l}\text { - Studies that exclusively enroll } \\
\text { subjects with chronic } \\
\text { conditions (e.g. hypertension, } \\
\text { diabetes) that are not related } \\
\text { to the index pregnancy } \\
\text { - Studies that exclusively enroll } \\
\text { subjects with a disease or } \\
\text { with the health outcome of } \\
\text { interest (intermediate or } \\
\text { endpoint health outcomes) } \\
\text { Studies done in hospitalized } \\
\text { or malnourished subjects, if } \\
\text { hospitalization is not related } \\
\text { to index pregnancy }\end{array}$ \\
\hline
\end{tabular}

Outcomes - Gestational age- and sex-specific birth weight

- Intermediate Outcomes:

- Fetal growth and growth velocities

- Intrauterine growth restriction (IUGR)

- Uterine artery or umbilical cord artery Doppler measurement 


\section{Search terms and electronic databases used}

\section{PubMed, US National Library of Medicine}

- Date(s) searched: January 1980 to January 2017

- Search Terms:

pregnancy[mh] OR "Prenatal Exposure Delayed Effects"[mesh] OR "Maternal Exposure"[mesh] OR "pregnant women"[mh] OR pregnan*[tiab] OR prenatal[tiab] OR maternal OR mother ${ }^{*}$ OR postpartum OR newborn*[tiab] OR perinatal OR peri-natal OR pre-conception OR preconception OR periconception OR periconception OR "Infant, Newborn"[Mesh] OR neonat*[tiab] OR newly born* OR "Peripartum Period"[Mesh] OR peripartum[tiab] OR peripartum[tiab] OR gestation* OR natal OR puerperium[tiab] OR "Maternal Nutritional Physiological Phenomena"[Mesh]

AND

hypertensi*[tiab] OR "Hypertension"[Mesh:NoExp] OR vomit* OR diabetes*[tiab] OR diabetic*[tiab] OR "Birth Weight"[Mesh] OR "Birth Weight"[tiab] OR "Glucose Intolerance"[Mesh] OR Glucose Intoleran*[tiab] OR glucose toleran* OR "Insulin Resistance"[Mesh] OR Insulin Resistan*[tiab] OR Dysglycemia[tiab] OR fasting blood glucose* OR "Hemoglobin A, Glycosylated"[Mesh] OR "Proteinuria"[Mesh:noexp] OR Albuminuria OR "Blood Pressure"[mh] OR "blood pressure"[tiab]

OR

"Diabetes, Gestational"[Mesh] OR (gestation*[tiab] AND (diabetes*[tiab] OR

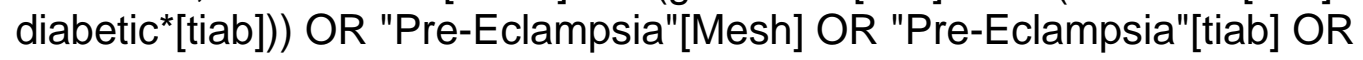
preeclampsia[tiab] OR "Hypertension, Pregnancy-Induced"[Mesh] OR Eclampsia OR "Gestational Age"[Mesh] OR "Morning Sickness"[Mesh] OR (Hyperemesis Gravidarum) OR "Gestational Age"[tiab] OR "Obstetric Labor, Premature"[Mesh] OR ((prematur* ${ }^{\star}[$ tiab] OR preterm [tiab]) AND (baby[tiab] OR infant*[tiab] OR birth OR labor OR membrane* OR babies)) OR "Fetal Growth Retardation"[Mesh] OR IUGR[tiab] OR "Intrauterine growth restriction" OR "Fetal Development"[Mesh:noexp] OR "Fetal Weight"[Mesh] OR "Umbilical Arteries"[Mesh] OR "Uterine Artery"[Mesh]

AND

("diet quality" OR dietary pattern* OR diet pattern* OR eating pattern* OR food pattern* OR eating habit* OR dietary habit* OR food habit* OR dietary profile* OR food profile* OR diet profile* OR eating profile* OR dietary guideline* OR dietary recommendation* OR eating style*) OR

(DASH[ti] OR DASH[tw] OR ("dietary approaches"[ti] AND hypertension[ti]) OR "Diet, Mediterranean"[Mesh] OR Mediterranean[ti] OR vegan* OR vegetarian* OR "Diet, Vegetarian"[Mesh] OR "prudent diet" OR "western diet" OR nordiet OR omni[ti] OR omniheart[tiab] OR (Optimal Macronutrient Intake Trial to Prevent Heart Disease) OR adventist* OR ((Okinawa* OR "Ethnic Groups"[Mesh] OR "plant based" OR Mediterranean[tiab] OR Nordic[tiab] OR "heart healthy"[tiab] OR indo-mediterranean) AND (diet[mh] OR diet[tiab] OR diets[tiab] OR 
food[mh]))) OR

("Guideline Adherence"[Mesh] AND (diet OR food OR eating OR eat OR dietary OR feeding OR nutrition OR nutrient*)) OR (adherence AND (nutrient ${ }^{\star}$ OR nutrition OR diet OR dietary OR food OR eat OR eating) AND (guideline* OR guidance OR recommendation*)) OR

(dietary score* OR adequacy index* OR kidmed OR Diet Quality Index* OR Food Score* OR Diet Score* OR MedDietScore OR Dietary Pattern Score* OR "healthy eating index") OR

((index*[ti] OR score*[ti] OR indexes OR scoring[ti] OR indices[ti]) AND (dietary[ti] OR nutrient*[ti] OR eating[tiab] OR food[ti] OR food[mh] OR diet[ti] OR $\operatorname{diet}[\mathrm{mh}])$ AND (pattern* OR habit* OR profile*)) OR meals[mh] OR meals[tiab] OR meal[tiab] OR mealtime ${ }^{\star}[$ tiab]

OR

$\operatorname{diet}[\mathrm{mh}: n o e x p]$ OR $\operatorname{diet}[\mathrm{ti}]$ OR $\operatorname{diets[ti]}$ OR food"[tiab] OR "Food"[mh:noexp] OR "Eating"[mh] OR dietary intake*[tiab] OR food intake*[tiab] OR food habits[mh] OR diet habit* ${ }^{\star}\left[\right.$ tiab] OR eating habit* ${ }^{*}$ tiab] OR food choice*[tiab] OR dietary choice*[tiab] OR dietary change*[tiab] NOT (editorial[ptyp] OR comment[ptyp] OR news[ptyp] OR letter[ptyp] OR review[ptyp] OR systematic[sb])

\section{Embase, Elsevier}

- Date(s) searched: January 1980 to January 2017

- Search Terms:

'pregnancy'/exp OR 'pregnant woman'/exp OR 'prenatal period'/exp OR 'mother'/exp OR 'prenatal exposure'/exp OR 'prenatal growth'/exp OR 'puerperium'/exp OR 'newborn'/exp OR prematurity/exp OR pregnan*:ti,ab OR maternal:ti,ab OR mother*:ti,ab OR prenatal:ti,ab OR pre-natal:ti,ab OR 'puerperium':ti,ab OR postpartum:ti,ab OR newborn:ti,ab OR neonat*:ti,ab OR "newly born":ti,ab OR periconception:ti,ab OR peri-conception:ti,ab OR preconception:ti,ab OR preconception;ti,ab OR gestation* OR peripartum:ti,ab OR peri-partum:ti,ab OR natal:ti,ab OR gestation* OR 'perinatal development'/exp OR 'perinatal care'/de OR perinatal:ti,ab OR peri-natal:ti,ab OR 'puerperium'/de OR 'puerperium':ti,ab OR 'maternal nutrition'/exp

AND

hypertensi* OR hyperemesis:ti,ab OR vomit*:ti,ab OR diabet* OR 'birth weight'/exp OR birthweight:ti,ab OR ((neonatal OR newborn) NEAR/3 weight)

OR

'glucose intolerance'/exp OR (Glucose NEAR/2 Intoleran*) OR (glucose NEAR/2 toleran*) OR 'insulin resistance'/exp OR (Insulin NEAR/1 Resistan*):ti,ab OR Dysglycemia OR "fasting blood glucose" OR 'hemoglobin A1c'/exp OR 'hemoglobin A1c' OR 'proteinuria'/exp OR albuminuria OR "Blood Pressure"/de OR

'pregnancy diabetes mellitus'/exp OR "diabetes mellitus gravidarum":ti,ab OR 
'eclampsia OR preeclampsia'/exp OR eclampsia:ti,ab OR preeclampsia:ti,ab OR pre-eclampsia:ti,ab OR 'maternal hypertension'/exp OR 'gestational age'/exp OR 'small for date infant'/exp OR 'gestational age' OR 'hyperemesis gravidarum'/exp OR 'morning sickness'/exp OR (gestation* NEAR/2 diabet $^{\star}$ ):ti,ab OR (Obstetric NEAR/3 (Labor OR labour)) OR (labor/exp AND

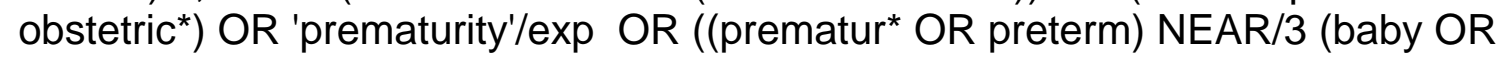
infant* OR babies OR birth OR childbirth OR labor OR membrane*)) OR 'intrauterine growth retardation'/de OR IUGR:ti,ab OR "Intrauterine growth restriction" OR 'fetus growth'/exp OR 'fetus development'/exp OR 'fetus weight'/exp OR 'umbilical artery'/exp OR 'uterine artery'/exp OR ((fetal OR fetus OR foetal OR foetus OR embryo*) NEAR/3 (weight OR develop* OR growth)):ti,ab

\section{AND}

'eating habit'/exp OR 'Mediterranean diet'/exp OR nordiet:ti,ab OR 'nordic diet':ti,ab OR DASH:ti,ab OR 'dietary approaches to stop hypertension':ti,ab OR vegan*:ab,ti OR vegetarian*:ab,ti OR 'vegetarian diet'/exp OR 'vegetarian'/exp OR 'prudent diet':ti,ab OR 'western diet':ti,ab OR 'Western diet'/exp OR meal/de OR omniheart:ti,ab OR omni:ti OR 'plant based diet' OR ((eating OR food OR diet $^{\star}$ OR calori ${ }^{\star}$ ) NEAR/3 (pattern? OR habit? OR profile? OR recommendation? OR guideline? OR style* OR choice* OR intake OR quality)) OR (('ethnic, racial and religious groups'/exp OR Okinawa* OR adventist* OR 'mediterranean') AND (diet/exp OR eating/exp OR 'food intake'/de OR calori* OR diet* OR food OR eating))

\section{OR}

Diet/de OR 'dietary intake'/de OR 'food preference'/de OR 'food intake'/de OR 'diet restriction'/exp OR 'eating habit'/exp OR diet*:ti OR kidmed:ab,ti OR 'meddietscore':ab,ti OR 'healthy eating index':ab,ti OR ((index OR score OR scoring OR indices) NEAR/3 (diet* OR eating OR food)) OR "food consumption"

OR

food $^{*}: t i, a b$ OR "Food"/de OR Eating:ti,ab OR (dietary NEAR/1 change*):ti,ab OR Meal*:ti,ab

\section{Cochrane Central Register of Controlled Trials, John Wiley \& Sons}

- Date(s) searched: January 1980 to January 2017

- Search Terms:

[mh pregnancy] OR [mh "Maternal Exposure"] OR [mh "Prenatal Exposure Delayed Effects"] OR [mh "pregnant women"] OR pregnan*:ti,ab OR prenatal OR maternal OR mother* OR postpartum OR newborn*:ti,ab OR perinatal OR perinatal OR pre-conception OR preconception OR peri-conception OR periconception OR [mh "Infant, Newborn"] OR neonat*:ti,ab OR (newly NEAR/1 born*) OR gestation* OR peripartum OR peri-partum OR natal:ti,ab OR puerperium OR gravidarum OR [mh "Peripartum Period"] OR peripartum:ti,ab OR peri-partum:ti,ab OR natal OR puerperium:ti,ab OR [mh "Maternal Nutritional 


\section{AND}

(hypertensi* $: t i, a b$ OR [mh ^Hypertension] OR vomit* ${ }^{\star}: t i, a b$ OR diabet* ${ }^{*}: t i, a b$ OR [mh "Birth Weight"] OR "Birth Weight":ti,ab OR [mh "Glucose Intolerance"] OR (Glucose NEAR/1 Intoleran*) OR (glucose NEAR/1 toleran*) OR [mh "Insulin Resistance"] OR (Insulin NEAR/1 Resistan*:ti,ab) OR Dysglycemia:ti,ab OR "fasting blood glucose" OR [mh "Hemoglobin A, Glycosylated"] OR [mh ^"Proteinuria"] OR Albuminuria OR [mh "Blood Pressure"] OR "blood pressure":ti,ab)

OR

[mh "Diabetes, Gestational"] OR (gestation* NEAR/1 diabet*) OR [mh "PreEclampsia"] OR "Pre-Eclampsia":ti,ab OR preeclampsia:ti,ab OR [mh "Hypertension, Pregnancy-Induced"] OR Eclampsia OR [mh "Gestational Age"] OR [mh "Morning Sickness"] OR (Hyperemesis NEAR/3 Gravidarum) OR "Gestational Age":ti,ab OR [mh "Birth Weight"] OR "Birth Weight":ti,ab OR ((neonatal OR newborn) NEAR/3 weight) OR [mh "Obstetric Labor, Premature"] OR ((prematur*:ti,ab OR preterm:ti,ab) AND (baby:ti,ab OR infant*:ti,ab OR birth OR labor OR membrane* OR babies)) OR [mh "Fetal Growth Retardation"] OR IUGR:ti,ab OR "Intrauterine growth restriction" OR [mh ^"Fetal Development"] OR [mh "Fetal Weight"] OR [mh "Umbilical Arteries"] OR [mh "Uterine Artery"]

AND (diet:ti OR diets:ti OR dietary:ti OR meal*:ti,ab OR "prudent diet" OR nordiet:ti,ab OR omniheart OR "Optimal Macronutrient Intake Trial to Prevent Heart Disease" OR ((Index OR score OR indices OR scoring) NEAR/3 (dietary OR diet OR food OR eating)) OR "adequacy index" OR kidmed OR MedDietScore)

OR 'dietary approaches to stop hypertension':ti,ab OR omniheart:ti,ab OR omni:ti OR 'plant based diet' OR ((eating OR food OR diet* OR calori*) NEAR/3 (pattern? OR habit? OR profile? OR recommendation? OR guideline? OR style* OR choice* OR intake OR quality))

OR

food $^{*}:$ ti,ab OR Eating:ti,ab OR (dietary NEAR/1 change*):ti,ab OR DASH:ti,ab OR vegan*:ab,ti OR vegetarian*:ab,ti OR omni:ti OR ((ethni* OR racial OR religio* OR asia* OR western OR Okinawa* OR adventist* OR 'mediterranean' OR Nordic* OR indo-mediterranean) NEAR/3 (calori* OR diet* OR food OR eating))

OR [mh "Diet, Mediterranean"] OR [mh "Diet, Vegetarian"] OR ([mh "Ethnic Groups"] AND ([mh diet] OR diet*:ti,ab OR [mh ^food] OR eat:ti,ab OR eating:ti,ab OR [mh "Eating"] OR [mh "food habits"])) OR

([mh "Guideline Adherence"] AND (diet OR food OR eating OR eat OR dietary)) OR ((adhere* OR adhering) AND (diet OR dietary OR food OR eat OR eating) AND (guideline* OR guidance OR recommendation*)) OR

[mh meals] OR [mh ^diet] OR diet*:ti,ab OR [mh ^"Food"] OR [mh "Eating"] OR [mh "food habits"] 


\section{CINAHL (Plus) with Full Text, EBSCO (Cumulative Index to Nursing and Allied Health Literature)}

- Date(s) searched: January 1980 to January 2017

- Search Terms:

(MH "Food and Beverages") OR (MH "Food") OR (MH "Diet") OR (MH "Eating") OR (MH "Eating Behavior") OR (MH "Meals+") OR (MH "Food Preferences") OR (MH "Food Habits") OR (MH "Mediterranean Diet") OR (MH "Diet, Western") OR (MH "DASH Diet") OR (MH "Vegetarianism")

OR meal* OR "prudent diet" OR nordiet OR omniheart OR "Optimal

Macronutrient Intake Trial to Prevent Heart Disease" OR ((Index OR score OR indices OR scoring) N3 (dietary OR diet OR food OR eating)) OR "adequacy index" OR kidmed OR MedDietScore

OR "dietary approaches to stop hypertension" OR "plant based diet" OR ((eating OR food* OR diet* OR calori ${ }^{\star}$ ) N3 (pattern? OR habit? OR profile? OR recommendation? OR guideline? OR style* OR choice* OR intake OR quality))

OR

(dietary NEAR/1 change $\left.{ }^{\star}\right)$ OR vegan* OR vegetarian* OR ((ethni* OR racial OR religio* OR asia* OR western OR Okinawa* OR adventist* OR 'mediterranean' OR Nordic* OR indo-mediterranean OR omni*) N3 (calori* OR diet* $^{\star}$ OR food OR eating))

OR (MH "Ethnic Groups+") AND ((mh diet) OR diet* OR (MH food) OR eat OR eating OR (MH "Eating") OR MH "food habits")) OR

((adhere* OR adhering) N3 (diet OR dietary OR food OR eat OR eating)) AND (guideline* OR guidance OR recommendation $\left.{ }^{\star}\right)$ )

(MH "Maternal Nutritional Physiology+") OR (MH "Maternal Exposure") OR (MH "Pregnancy+") OR (MH "Pregnancy in Adolescence+") OR (MH "Maternal Age 14 and Under") OR (MH "Pregnancy Outcomes") OR (MH "Mothers+") OR (MH "Prenatal Nutritional Physiology") OR (MH "Infant, Newborn+") OR (MH "Postnatal Period+") OR (MH "Periconceptual Period")

AND

(MH "Hypertension+") OR (MH "Nausea and Vomiting+") OR (MH "Vomiting+") $\mathrm{OR}$ (MH "Birth Weight") OR (MH "Glucose Tolerance Test") OR (MH "Prediabetic State") OR (MH "Glucose Intolerance") OR (MH "Insulin Resistance+") OR (MH "Blood Pressure+") OR (MH "Proteinuria+") OR (MH "Hemoglobin A, Glycosylated")

OR

(MH "Diabetes Mellitus, Gestational") OR (MH "Gestational Age") OR (MH "PreEclampsia+") OR (MH "Eclampsia+") OR (MH "Fetal Growth Retardation") OR (MH "Fetal Weight") OR (MH "Umbilical Arteries") OR (MH "Delivery, Obstetric+")

Limiters - Published Date: 19800101-; Peer Reviewed; English Language; 
Exclude MEDLINE records; Pregnancy

Narrow by SubjectMajor: - energy intake

Narrow by SubjectMajor: - vegetarianism

Narrow by SubjectMajor: - women's health

Narrow by SubjectMajor: - pregnancy outcomes

Narrow by SubjectMajor: - pregnancy complications

Narrow by SubjectMajor: - food habits

Narrow by SubjectMajor: - diabetes mellitus, gestational 
Figure 2: Flow chart of literature search and screening results

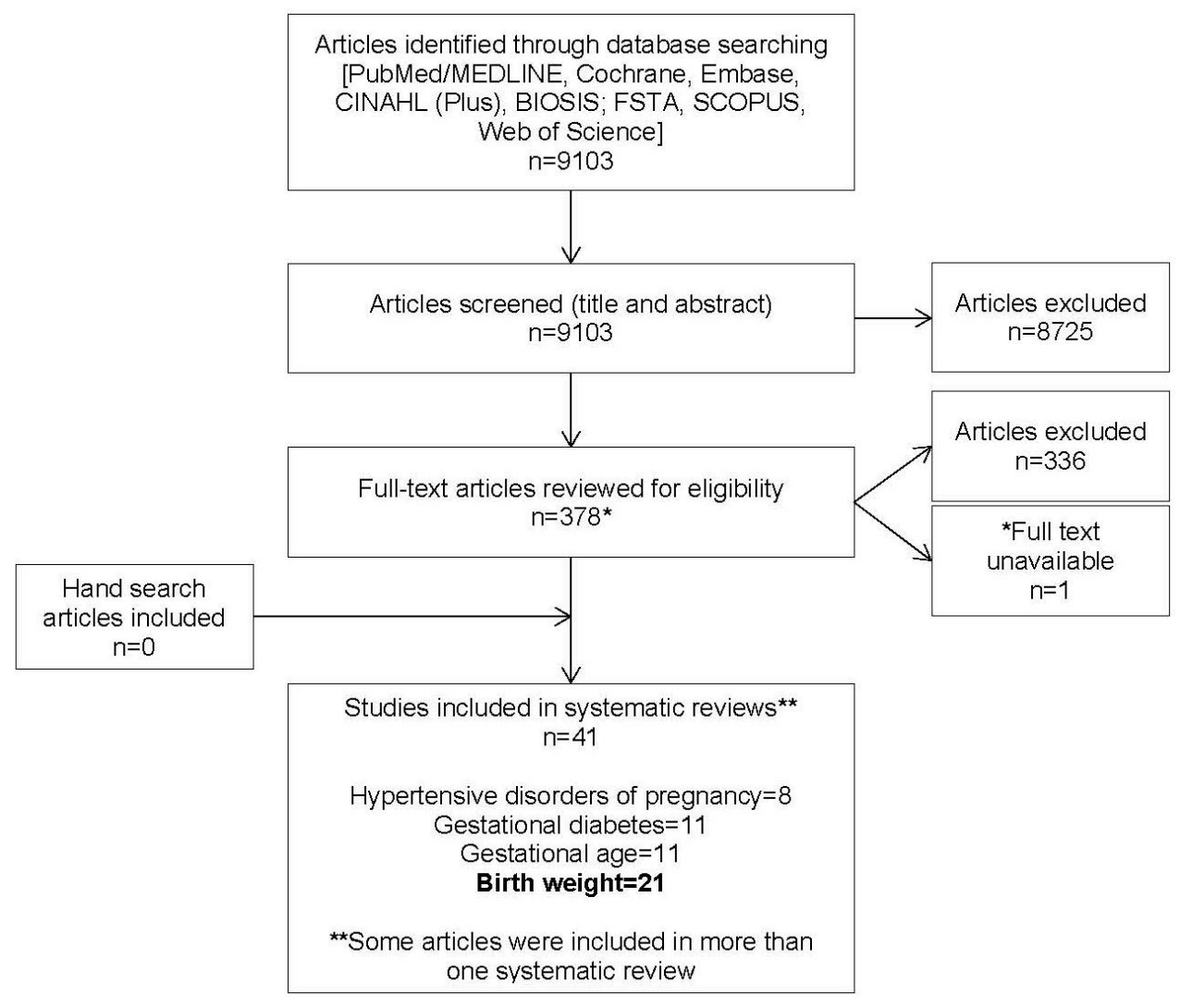

This flow chart illustrates the literature search and screening results for articles examining the relationship between dietary patterns before and during pregnancy and gestational age- and sex-specific birth weight. The results of the electronic database searches were screened independently by two NESR analysts in a step-wise manner by reviewing titles, abstracts, and full text articles to determine which articles met the criteria for inclusion. A manual search was done to ascertain articles not identified through the electronic database search. The systematic review on the relationship between dietary patterns before and during pregnancy and gestational age- and sexspecific birth weight included 21 articles. The literature search was conducted for multiple systematic reviews that addressed the relationships between dietary patterns before and during pregnancy on gestational age- and sex-specific birth weight, hypertensive disorders of pregnancy, gestational diabetes mellitus, and gestational age at birth; the systematic reviews on hypertensive disorders of pregnancy, gestational diabetes mellitus, and gestational age at birth are reported elsewhere. 


\section{List of excluded articles}

The table below lists the excluded articles with at least one reason for exclusion, but may not reflect all possible reasons.

Table 8: List of excluded articles

\begin{tabular}{|c|c|c|}
\hline & Excluded Citations & Reasons for Exclusion \\
\hline 1 & $\begin{array}{l}\text { Aaltonen, J, Ojala, T, Laitinen, K et al. Risk Reduction of Infant } \\
\text { Insulin Resistance by Dietary Intervention during Pregnancy and } \\
\text { Breastfeeding. Pediatric Academic Societies Annual Meeting; } 2009 \\
\text { May } 2 \text { 5; Baltimore MD, United States, } 2009\end{array}$ & Dependent variable \\
\hline 2 & $\begin{array}{l}\text { Abel, Ht, Bannert, N, Starke, I et al. Study into Ca/P homeostasis } \\
\text { in premature babies on different diets. Klin Padiatr, 1991, } 203\end{array}$ & Independent variable \\
\hline 3 & $\begin{array}{l}\text { Adami, G. F., Friedman, D., Cuneo, S. et al. Intravenous nutritional } \\
\text { support in pregnancy. Experience following biliopancreatic } \\
\text { diversion. Clinical Nutrition, 1992, 11: 106-109 }\end{array}$ & Independent variable \\
\hline 4 & $\begin{array}{l}\text { Akbari, Z., Mansourian, M., Kelishadi, R. Relationship of the intake } \\
\text { of different food groups by pregnant mothers with the birth weight } \\
\text { and gestational age: Need for public and individual educational } \\
\text { programs. J Educ Health Promot, } 2015,4 \text {. PMID:25883993. }\end{array}$ & Independent variable \\
\hline 5 & $\begin{array}{l}\text { Alfonso, H. Preventing preeclampsia: the evidence on nutrients. } \\
\text { Nurs Womens Health, 2009, 13: 419-21. PMID:19821918. }\end{array}$ & Study design \\
\hline 6 & $\begin{array}{l}\text { Ali, H. I., Jarrar, A. H., El Sadig, M. et al. Diet and carbohydrate } \\
\text { food knowledge of multi-ethnic women: a comparative analysis of } \\
\text { pregnant women with and without Gestational Diabetes Mellitus. } \\
\text { PLoS One, } 2013,8 \text {. PMID:24069200. }\end{array}$ & Study design \\
\hline 7 & $\begin{array}{l}\text { Alwan, N. A., Greenwood, D. C., Simpson, N. A. et al. Dietary iron } \\
\text { intake during early pregnancy and birth outcomes in a cohort of } \\
\text { British women. Hum Reprod, 2011, 26: 911-9. PMID:21303776. }\end{array}$ & Independent variable \\
\hline 8 & $\begin{array}{l}\text { Andreasyan, K., Ponsonby, A. L., Dwyer, T. et al. Higher maternal } \\
\text { dietary protein intake in late pregnancy is associated with a lower } \\
\text { infant ponderal index at birth. Eur J Clin Nutr, 2007, 61: 498-508. } \\
\text { PMID:17136041. }\end{array}$ & Independent variable \\
\hline
\end{tabular}




\section{Excluded Citations}

$9 \quad$ Arkkola, T., Uusitalo, U., Kronberg-Kippila, C. et al. Seven distinct dietary patterns identified among pregnant Finnish women-associations with nutrient intake and sociodemographic factors. Public Health Nutr, 2008, 11: 176-82. PMID:17610760.

10 Asaka, A., Imaizumi, Y., Inouye, E. Analysis of multiple births in Japan. V. Effects of gestational age, maternal age and other factors on growth rate of weight in twins. Jinrui Idengaku Zasshi, 1981, 26: 83-90. PMID:7328851.

11 Asbee, Sm, Jenkins, Tr, Butler, Jr et al. Dietary counseling prevents excessive weight gain during pregnancy, a randomized controlled trial. Obstet Gynecol, 2008, 111

12 Asp, N. G. Nutrition and human development. Scandinavian Journal of Food and Nutrition, 2006, 50

13 Babson, Sg, Bramhall, Jl. Diet and growth in the premature infant. Journal of Pediatrics, 1969, 74: 890-900

14 Bakouei, S., Reisian, F., Lamyian, M. et al. High Intake of Manganese During Second Trimester, Increases the Risk of Preterm Delivery: A Large Scale Cohort Study. Glob J Health Sci, 2015, 7: 226-32. PMID:26156900.

15 Bao, W., Bowers, K., Tobias, D. K. et al. Prepregnancy lowcarbohydrate dietary pattern and risk of gestational diabetes mellitus: a prospective cohort study. Am J Clin Nutr, 2014, 99: 1378-84. PMID:24717341.

16 Bao, W., Li, S., Chavarro, J. E. et al. Low Carbohydrate-Diet Scores and Long-term Risk of Type 2 Diabetes Among Women With a History of Gestational Diabetes Mellitus: A Prospective Cohort Study. Diabetes Care, 2016, 39: 43-9. PMID:26577416.

Bao, W., Tobias, D. K., Hu, F. B. et al. Pre-pregnancy potato

\section{Reasons for Exclusion}

Dependent variable

Independent variable

Dependent variable

Independent variable, study design

Date

Independent variable

\author{
Independent variable
}

Independent variable

Dependent variable consumption and risk of gestational diabetes mellitus: prospective cohort study. Bmj, 2016, 352. PMID:26759275. 


\section{Excluded Citations}

\section{Reasons for Exclusion}

18 Bao, W., Tobias, D. K., Olsen, S. F. et al. Pre-pregnancy fried food Independent variable consumption and the risk of gestational diabetes mellitus: a prospective cohort study. Diabetologia, 2014, 57: 2485-91. PMID:25303998.

19 Baron, R., Te Velde, S. J., Heymans, M. W. et al. The Independent variable Relationships of Health Behaviour and Psychological Characteristics with Spontaneous Preterm Birth in Nulliparous Women. Matern Child Health J, 2016, . PMID:27581004.

20 Bell, E. H., Geyer, J., Jones, L. A structured intervention improves breastfeeding success for ill or preterm infants. MCN Am J Matern Child Nurs, 1995, 20: 309-14. PMID:8551932.

21 Berntorp, K. E. Gestational diabetes: what's up?. Diabetologia, Study design 2016, 59: 1382-1384

22 Bertolotto, A., Volpe, L., Calianno, A. et al. Physical activity and dietary habits during pregnancy: effects on glucose tolerance. $\mathrm{J}$ Matern Fetal Neonatal Med, 2010, 23: 1310-4. PMID:20334531.

23 Bhatia, B. D., Banerjee, D., Agarwal, D. K. et al. Fetal growth: relationship with maternal dietary intakes. Indian J Pediatr, 1983, 50: 113-20. PMID:6618569.

24 Bjerregaard, P., Hansen, J. C. Effects of smoking and marine diet Independent variable on birthweight in Greenland. Arctic Med Res, 1996, 55: 156-64. PMID:9115541.

25 Bloomfield, F. H., Oliver, M. H., Hawkins, P. et al. A Independent variable, health status periconceptional nutritional origin for noninfectious preterm birth. Science, 2003, 300. PMID:12714735.

26 Bo, S., Rosato, R., Ciccone, G. et al. Simple lifestyle recommendations and the outcomes of gestational diabetes. A 2 x 2 factorial randomized trial. Diabetes Obes Metab, 2014, 16: 10325. PMID:24646172. 


\section{Excluded Citations}

27 Bobinski, R., Mikulska, M., Mojska, H. et al. Assessment of the diet components of pregnant women as predictors of risk of preterm birth and born baby with low birth weight. Ginekol Pol, 2015, 86: 292-9. PMID:26117989.

28 Bobinski, R., Mikulska, M., Mojska, H. et al. The Dietary Composition of Women Who Delivered Healthy Full-Term Infants, Preterm Infants, and Full-Term Infants Who Were Small for Gestational Age. Biol Res Nurs, 2015, 17: 495-502. PMID:25358685.

29 Borberg, C., Gillmer, M. D., Brunner, E. J. et al. Obesity in pregnancy: the effect of dietary advice. Diabetes Care, 1980, 3: 476-81. PMID:6993162.

30 Borgen, I., Aamodt, G., Harsem, N. et al. Maternal sugar consumption and risk of preeclampsia in nulliparous Norwegian women. Eur J Clin Nutr, 2012, 66: 920-5. PMID:22713766.

31 Bower, D. The influence of dietary salt intake on pre-eclampsia. Journal of obstetrics and gynaecology of the British Commonwealth, 1961, 63: 123-6

32 Bowers, K., Tobias, D. K., Yeung, E. et al. A prospective study of prepregnancy dietary fat intake and risk of gestational diabetes. Am J Clin Nutr, 2012, 95: 446-53. PMID:22218158.

33 BrantsÃ particularly in pregnancy â $\epsilon^{\prime \prime}$ Results from MoBa studies of maternal diet and pregnancy outcomes. Norsk Epidemiologi, 2014, 24: 63-77

34 Brantsaeter, A. L., Myhre, R., Haugen, M. et al. Intake of probiotic food and risk of preeclampsia in primiparous women: the Norwegian Mother and Child Cohort Study. Am J Epidemiol, 2011, 174: 807-15. PMID:21821542.

35 Breslow, S, Belafsky, Ha, Shangold, Je et al. Control of weight gain in pregnancy: double blind study of a dieting aid. Clinical medicine, 1963, 70: 931-8

\section{Reasons for Exclusion}

Independent variable, study design

Independent variable, study design

Independent variable

Independent variable

Date

Independent variable

Study design

Independent variable

Date 


\section{Excluded Citations}

\section{Reasons for Exclusion}

36 Brooke, O. G. Low birth weight babies. Nutrition and feeding. Br J Dependent variable Hosp Med, 1982, 28: 462-9. PMID:7171896.

37 Brooke, O. G. Nutrition in the preterm infant. Lancet, 1983, 1:514- Study subjects 6. PMID:6131220.

38 Brown, J. E., Kahn, E. S., Hartman, T. J. Profet, profits, and proof: Independent variable do nausea and vomiting of early pregnancy protect women from harmful vegetables?. Am J Obstet Gynecol, 1997, 176: 179-81. PMID:9024110.

$39 \quad$ Brumfield, C. G., Huddleston, J. F. The management of diabetic ketoacidosis in pregnancy. Clin Obstet Gynecol, 1984, 27: 50-9. PMID:6423330.

40 Bruno, R., Petrella, E., Bertarini, V. et al. Adherence to a lifestyle programme in overweight/obese pregnant women and effect on gestational diabetes mellitus: a randomized controlled trial. Matern Child Nutr, 2016, . PMID:27647837.

41 Buchanan, T. A., Kjos, S. L. Diabetes and pregnancy. Curr Ther Study design Endocrinol Metab, 1994, 5: 278-83. PMID:7704732.

42 Buul, E, Rijpkema, A, Steegers, E et al. Chronic dietary sodium Independent variable restriction in pregnancy reduces calcium intake. J Perinat Med, 1992, 20

43 Campbell, Dm. Dietary restriction in obesity and its effect on Not peer-reviewed neonatal outcome. Nutrition in Pregnancy. Proceedings of 10th Study Group of the Rcog; 1983; London, UK, 1983, : 243-50

44 Canda, M. T., Sezer, O., Demir, N. An audit of seafood consumption awareness during pregnancy and its association with maternal and fetal outcomes in a Turkish population. J Obstet Gynaecol, 2011, 31: 293-7. PMID:21534748.

45 Carmichael, S. L., Yang, W., Shaw, G. M. Maternal dietary nutrient intake and risk of preterm delivery. Am J Perinatol, 2013, 30: 57988. PMID:23208764. 


\section{Excluded Citations}

46 Carter, J. P., Furman, T., Hutcheson, H. R. Preeclampsia and reproductive performance in a community of vegans. South Med J, 1987, 80: 692-7. PMID:3589760.

47 Carver, Jd, Saste, Md, Sosa, R et al. Dietary nucleotide (NT) effects on superior mesenteric artery (SMA) blood flow in preterm infants. Pediatr Res, 2000, 47 C'De Baca, J., Lapham, S. C., Skipper, B. J. et al. Use of
computer interview data to test associations between risk factors
and pregnancy outcomes. Comput Biomed Res, 1997, 30: 232-43. PMID:9281330.

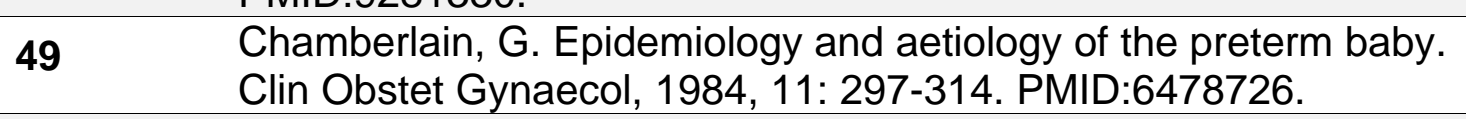

50 Chandler-Laney, P. C., Schneider, C. R., Gower, B. A. et al. Association of late-night carbohydrate intake with glucose tolerance among pregnant African American women. Matern Child Nutr, 2016, 12: 688-98. PMID:25786515. Chavarro, J. E., Halldorsson, T. I., Leth, T. et al. A prospective
study of trans fat intake and risk of preeclampsia in Denmark. Eur J
Clin Nutr, 2011, 65: 944-51. PMID:21559043.

52 Chen, C. M., Weng, H. C., Li, Y. C. et al. The evaluation of dietary Language intervention on the blood glucose level of gestational diabetes mellitus pregnant women. Nutritional Sciences Journal, 1999, 24: 250-261

\footnotetext{
53 Chen, L., Hu, F. B., Yeung, E. et al. Prospective study of pregestational diabetes mellitus. Diabetes Care, 2009, 32: 2236-41. PMID:19940226.

54 Chong, M. F., Chia, A. R., Colega, M. et al. Maternal Protein Intake during Pregnancy Is Not Associated with Offspring Birth Weight in a Multiethnic Asian Population. J Nutr, 2015, 145: 1303-

Independent variable 10. PMID:25948786.

Independent variable
}

Independent variable

Independent variable

Reasons for Exclusion

Independent variable

Study design

Independent variable

Independent variable 


\section{Excluded Citations}

55 Christian, K, Andreas, M, Martin, F. Diet and lifestyle modification in mothers with burnout syndrome: Ayurvedic versus conventional standard counselling-design of a randomised clinical pilot study (VEDA-Trial) [abstract]. European journal of integrative medicine [abstracts of the 5th european congress for integrative medicine; 2012 sept 21-22; flo, 2012, 4: 47-8

56 Clapp, J. F. Effects of Diet and Exercise on Insulin Resistance during Pregnancy. Metab Syndr Relat Disord, 2006, 4: 84-90. PMID:18370754.

57 Clausen, T., Slott, M., Solvoll, K. et al. High intake of energy, sucrose, and polyunsaturated fatty acids is associated with increased risk of preeclampsia. Am J Obstet Gynecol, 2001, 185: 451-8. PMID:11518908.

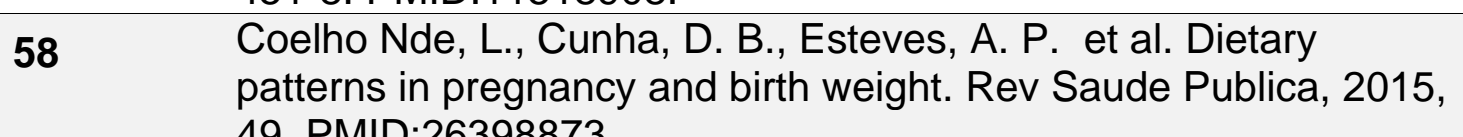
49. PMID:26398873.

\begin{tabular}{|c|c|c|}
\hline I & $\begin{array}{l}\text { sucrose, and polyunsaturated fatty acids is associated with } \\
\text { increased risk of preeclampsia. Am J Obstet Gynecol, 2001, 185: } \\
\text { 451-8. PMID:11518908. }\end{array}$ & \\
\hline 58 & $\begin{array}{l}\text { Coelho Nde, L., Cunha, D. B., Esteves, A. P. et al. Dietary } \\
\text { patterns in pregnancy and birth weight. Rev Saude Publica, 2015, } \\
\text { 49. PMID:26398873. }\end{array}$ & Study design \\
\hline 59 & $\begin{array}{l}\text { Cooney, G. Food for thought. Midwives, 2008, 11: 30-1. } \\
\text { PMID:24902215. }\end{array}$ & Study design \\
\hline 60 & $\begin{array}{l}\text { Cooper, M. L. Stories to learn from: toxemia in pregnancy. } \\
\text { Midwifery Today Int Midwife, 2014, : 18-21. PMID:25980103. }\end{array}$ & Not peer-reviewed \\
\hline 61 & $\begin{array}{l}\text { Corbett, M. A., Burst, H. V. Nutritional intervention in pregnancy. J } \\
\text { Nurse Midwifery, 1983, 28: 23-9. PMID:6554311. }\end{array}$ & Study design, independent variable \\
\hline 62 & $\begin{array}{l}\text { Cosgrove, M., Davies, D. P. Poor diet in pregnancy may be a proxy } \\
\text { for some other hostile influence on fetal growth [8]. Br Med J, 1996, } \\
312: 1478-1479\end{array}$ & Independent variable, study design \\
\hline 63 & $\begin{array}{l}\text { Costa-Orvay, Ja, Figueras-Aloy, J, Romera, G et al. The effects of } \\
\text { varying protein and energy intakes on the growth and body } \\
\text { composition of very low birth weight infants. Nutr J, 2011, } 10\end{array}$ & Independent variable \\
\hline 64 & $\begin{array}{l}\text { Crozier, S. R., Inskip, H. M., Godfrey, K. M. et al. Nausea and } \\
\text { vomiting in early pregnancy: Effects on food intake and diet quality. } \\
\text { Matern Child Nutr, 2016, . PMID:27896913. }\end{array}$ & Dependent variable \\
\hline
\end{tabular}

\section{Reasons for Exclusion}

Dependent variable

Study design

Independent variable

Study design

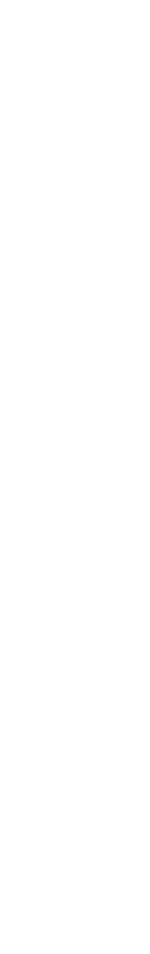




\section{Excluded Citations}

65 Dancause, K. N., Mutran, D., Elgbeili, G. et al. Dietary change mediates relationships between stress during pregnancy and infant head circumference measures: the QF2011 study. Matern Child Nutr, 2016, . PMID:27562643.

66 Darling, A. M., Mitchell, A. A., Werler, M. M. Preconceptional Iron Intake and Gestational Diabetes Mellitus. Int J Environ Res Public Health, 2016, 13. PMID:27231921.

67 Davidson, J. K. Newer approaches to diet management of diabetes: calorie control. Med Times, 1980, 108: 35-40. PMID:7374404.

68 Davies, W. E., Hopkins, P. C., Rose, S. J. et al. The influence of different taurine diets on hearing development in normal babies. A preliminary report. Adv Exp Med Biol, 1996, 403: 631-7. PMID:8915404.

69 Davison, J. M., Lindheimer, M. D. Pregnancy in renal transplant recipients. J Reprod Med, 1982, 27: 613-21. PMID:6757420.

70 Dawn, Cs. Effects of substandard prenatal diet and nutrition on the Date development and incidence of pre-eclampsia of pregnancy. $\mathrm{J}$ Obstet Gynaecol India, 1961, 12: 237-45

\section{1 de Seymour, J., Chia, A., Colega, M. et al. Maternal Dietary} Patterns and Gestational Diabetes Mellitus in a Multi-Ethnic Asian Cohort: The GUSTO Study. Nutrients, 2016, 8. PMID:27657116.

72 Deka, D., Sharma, N. Nutrition in pregnancy and lactation. Perinatology, 2005, 7: 1-15

73 Delemarre, F. M., van Leest, L. A., Jongsma, H. W. et al. Effect of low-sodium diet on uteroplacental circulation. J Matern Fetal Med, 2000, 9: 197-200. PMID:11048827.

74 Demmelmair, H, Klingler, M, Campoy, $\mathrm{C}$ et al. The influence of habitual diet and increased docosahexaenoic acid intake during pregnancy on the fatty acid composition of individual placental lipids [Study design]. J Pediatr Gastroenterol Nutr, 2005, 40: 622-3

\section{Reasons for Exclusion}

Independent variable

Independent variable

Study design

Independent variable

Health status

Study design

Study design

Independent variable

Study design 


\section{Excluded Citations}

75 Deveer, R., Deveer, M., Akbaba, E. et al. The effect of diet on pregnancy outcomes among pregnant with abnormal glucose challenge test. Eur Rev Med Pharmacol Sci, 2013, 17: 1258-61. PMID:23690197.

76 Dieckmann, Wj, Davis, Me, Rynkiewicz, Lm et al. Does the administration of diethylstilbestrol during pregnancy have therapeutic value?. Am J Obstet Gynecol, 1953, 66: 1062-75

77 Diet \& nutrition. Good news: caffeine in pregnancy doesn't affect the baby's growth..and folic acid seems to prevent cleft lip. Child Health Alert, 2007, 25: 5-6. PMID:17443983.

78 Dodd, J. M., Deussen, A. R., Mohamad, I. et al. The effect of antenatal lifestyle advice for women who are overweight or obese on secondary measures of neonatal body composition: The LIMIT randomised trial. BJOG: An International Journal of Obstetrics and Gynaecology, 2016, 123: 244-253

79 Dodd, J. M., McPhee, A. J., Turnbull, D. et al. The effects of antenatal dietary and lifestyle advice for women who are overweight or obese on neonatal health outcomes: the LIMIT randomised trial. BMC Med, 2014, 12. PMID:25315325.

80 Dominguez, L. J., Martinez-Gonzalez, M. A., Basterra-Gortari, F. J. Independent variable et al. Fast food consumption and gestational diabetes incidence in the SUN project. PLoS One, 2014, 9. PMID:25215961.

81 Donnelly, J, Horan, M, Walsh, J et al. Impact of a Low Gl Diet on Not peer-reviewed Neonatal Body Composition [ROLO Kids]. Pediatric Academic Societies Annual Meeting, 2013,

82 Donnelly, J. M., Walsh, J. M., Byrne, J. et al. Impact of maternal diet on neonatal anthropometry: a randomized controlled trial. Pediatr Obes, 2015, 10: 52-6. PMID:24443392.

\section{Reasons for Exclusion}

Independent variable

Date

Not peer-reviewed

Independent variable

Independent variable

Independent variable

Doyle, W. Maternal nutrition and low birth weight. J Fam Health Care, 2002, 12. PMID:12630147.
Study design 


\section{Excluded Citations}

\section{Reasons for Exclusion}

84 Doyle, W., Crawford, M. A., Wynn, A. H. A. et al. Maternal nutrient Independent variable intake and birth-weight. Journal of Human Nutrition and Dietetics, 1989, 2: 415-422

85 Drake, A. J., McPherson, R. C., Godfrey, K. M. et al. An unbalanced maternal diet in pregnancy associates with offspring epigenetic changes in genes controlling glucocorticoid action and foetal growth. Clin Endocrinol (Oxf), 2012, 77: 808-15. PMID:22642564.

86 Drouillet, P., Kaminski, M., De Lauzon-Guillain, B. et al. Association between maternal seafood consumption before pregnancy and fetal growth: evidence for an association in overweight women. The EDEN mother-child cohort. Paediatr Perinat Epidemiol, 2009, 23: 76-86. PMID:19228317.

87 Dubois, S., Coulombe, C., Pencharz, P. et al. Ability of the Higgins Independent variable Nutrition Intervention Program to improve adolescent pregnancy outcome. J Am Diet Assoc, 1997, 97: 871-8. PMID:9259709.

88 Dunn, C., Kolasa, K., Dunn, P. C. et al. Dietary intake of pregnant Independent variable, dependent variable adolescents in a rural southern community. J Am Diet Assoc, 1994, 94: 1040-1. PMID:8071488.

89 Ebbs, Jh, Tisdall, Ff, Scott, Wa. The influence of prenatal diet on Date the mother and child. Journal of Nutrition, 1941, 22: 515-26

$90 \quad$ Elmacioglu, F., Surucu, B., Alper, T. et al. Is adequate and balanced nutrition during pregnancy more effective than iron and folic acid supplements?. Central European Journal of Medicine, 2010, 5: 235-242

91 Ershoff, Dh, Aaronson, Nk, Danaher, Bg et al. Behavioral, health, Independent variable and cost outcomes of an HMO based prenatal health education program. Public health reports, 1983, 98: 536-47

92 Ershoff, D. H., Aaronson, N. K., Danaher, B. G. et al.. Behavioral, Duplicate health, and cost outcomes of an HMO-based prenatal health education program. Public Health Rep, 1983, 98: 536-47. PMID:6419268. 


\section{Excluded Citations}

93 Eshriqui, I., Vilela, A. A., Rebelo, F. et al. Gestational dietary patterns are not associated with blood pressure changes during pregnancy and early postpartum in a Brazilian prospective cohort. Eur J Nutr, 2016, 55: 21-32. PMID:25526968.

94 Fairburn, C. G., Stein, A., Jones, R. Eating habits and eating disorders during pregnancy. Psychosom Med, 1992, 54: 665-72. PMID:1454960.

95 Farbu, J., Haugen, M., Meltzer, H. M. et al. Impact of singlehood during pregnancy on dietary intake and birth outcomes- a study in the Norwegian Mother and Child Cohort Study. BMC Pregnancy Childbirth, 2014, 14. PMID:25475509.

96 Fard, N Mehrabian F Sarraf-Zadegan NS. Fat-modified diets during Country pregnancy and lactation and serum lipids after birth. Indian $\mathrm{J}$ Pediatr, 2004, 71: 683-7

97 Farland, L. V., Rifas-Shiman, S. L., Gillman, M. W. Early Pregnancy Cravings, Dietary Intake, and Development of Abnormal Glucose Tolerance. J Acad Nutr Diet, 2015, 115. PMID:26099686.

98 Ferland, S., O'Brien, H. T. Maternal dietary intake and pregnancy outcome. J Reprod Med, 2003, 48: 86-94. PMID:12621791.

99 Flynn, A. C., Seed, P. T., Patel, N. et al. Dietary patterns in obese pregnant women; influence of a behavioral intervention of diet and physical activity in the UPBEAT randomized controlled trial. Int $\mathrm{J}$ Behav Nutr Phys Act, 2016, 13. PMID:27894316.

100 Ford, J. H. Preconception risk factors and SGA babies: Papilloma virus, omega 3 and fat soluble vitamin deficiencies. Early Hum Dev, 2011, 87: 785-9. PMID:21705161.

101 Fowles, E. R., Gabrielson, M. First trimester predictors of diet and birth outcomes in low-income pregnant women. J Community Health Nurs, 2005, 22: 117-30. PMID:15877540.

102 Fraser, R. B., Ford, F. A., Milner, R. D. G. A controlled trial of a high dietary fibre intake in pregnancy-effects in plasma glucose and insulin levels. Diabetologia, 1983, 25: 238-241

\section{Reasons for Exclusion}

Dependent variable

Independent variable

Independent variable

Study design, independent variable

Independent variable

Health status

Independent variable

Independent variable, study design

Independent variable 


\section{Excluded Citations}

103 Fraser, Rb. High fibre diets in pregnancy. Nutrition in Pregnancy. Proceedings of 10th Study Group of the Royal College of Obstetricians and Gynaecologists; 1982 September, 1983, : 26980

104 Garratt, F. N. Pre-eclampsia: a challenge to public health teams worldwide to ensure that maternal diets contain adequate levels of folic acid, n3 polyunsaturated fatty acids and vitamin $\mathrm{D}$ at conception. Public Health, 2009, 123: 95-6. PMID:19058819.

105 Gennaro, S., Biesecker, B., Fantasia, H. C. et al. Nutrition profiles of African [corrected] American women in the third trimester. MCN Am J Matern Child Nurs, 2011, 36: 120-6. PMID:21350375.

106 Gerrard, J., Popeski, D., Ebbeling, L. et al. Dietary omega 3 fatty acids and gestational hypertension in the Inuit. Arctic Med Res, 1991, : 763-7. PMID:1365294.

107 Gesteiro, E., Rodriguez Bernal, B., Bastida, S. et al. Maternal diets Dependent variable with low healthy eating index or Mediterranean diet adherence scores are associated with high cord-blood insulin levels and insulin resistance markers at birth. Eur J Clin Nutr, 2012, 66: 100815. PMID:22828732.

\begin{tabular}{lll}
\hline 108 & $\begin{array}{l}\text { Ghebremeskel, K., Leighfield, M., Ashwell, M. et al. Infant brain } \\
\text { lipids and diet [1]. Lancet, 1992, 340: 1093-1094 }\end{array}$ & Dependent variable \\
\hline 109 & $\begin{array}{l}\text { Gillen, L., Tapsell, L. C., Martin, G. S. et al. The type and } \\
\text { frequency of consumption of carbohydrate-rich foods may play a } \\
\text { role in the clinical expression of insulin resistance during } \\
\text { pregnancy. Dietetics, 2002, 59: 135-143 }\end{array}$ & Dependent variable \\
\hline $\mathbf{1 1 0}$ & $\begin{array}{l}\text { Glueck, C. J., Goldenberg, N., Pranikoff, J. et al. Effects of } \\
\text { metformin-diet intervention before and throughout pregnancy on } \\
\text { obstetric and neonatal outcomes in patients with polycystic ovary } \\
\\
\text { syndrome. Curr Med Res Opin, 2013, 29: 55-62. PMID:23205605. }\end{array}$ & Health status \\
\hline $\mathbf{1 1 1}$ & $\begin{array}{l}\text { Godfrey, K., Robinson, S., Barker, D. J. et al. Maternal nutrition in } \\
\text { early and late pregnancy in relation to placental and fetal growth. } \\
\text { Bmj, 1996, 312: 410-4. PMID:8601112. }\end{array}$ \\
\hline
\end{tabular}

\section{Reasons for Exclusion}

Independent variable, not peer reviewed

Study design

Independent variable, dependent variable

Independent variable, study design

\begin{tabular}{l} 
on \\
not peer reviewed \\
\hline dependent variable \\
\hline study design
\end{tabular} 


\section{Excluded Citations}

112 Grant, S. M., Wolever, T. M., O'Connor, D. L. et al. Effect of a low glycaemic index diet on blood glucose in women with gestational hyperglycaemia. Diabetes Res Clin Pract, 2011, 91: 15-22.

PMID:21094553.

113 Gray-Donald, K., Robinson, E., Collier, A. et al. Intervening to reduce weight gain in pregnancy and gestational diabetes mellitus in Cree communities: an evaluation. Cmaj, 2000, 163: 1247-51. PMID:11107459.

114 Grivell, R. M., Yelland, L. N., Deussen, A. et al. Antenatal dietary and lifestyle advice for women who are overweight or obese and the effect on fetal growth and adiposity: the LIMIT randomised trial. Bjog, 2016, 123: 233-43. PMID:26841216.

115 Guilloty, N. I., Soto, R., Anzalota, L. et al. Diet, Pre-pregnancy BMI, and Gestational Weight Gain in Puerto Rican Women. Matern Child Health J, 2015, 19: 2453-61. PMID:26100133.

116 Guldner, L., Monfort, C., Rouget, F. et al. Maternal fish and Independent variable shellfish intake and pregnancy outcomes: a prospective cohort study in Brittany, France. Environ Health, 2007, 6. PMID:17958907.

117 Gupta, A. P., Bhandari, B., Gupta, A. et al. Stool pH and sugar in Independent variable preterm neonates. Indian J Pediatr, 1984, 51: 391-3. PMID:6526446.

118 Haas, A. V. Diet du jour! Pregnancy and popular diets. Midwifery Study design, non peer-reviewed Today Int Midwife, 2014, : 53-5. PMID:25975083.

119 Haggarty, P., Campbell, D. M., Duthie, S. et al. Diet and deprivation in pregnancy. Br J Nutr, 2009, 102: 1487-97. PMID:19682400.

120 Halldorsson, T. I., Thorsdottir, I., Meltzer, H. M. et al. Dioxin-like activity in plasma among Danish pregnant women: dietary predictors, birth weight and infant development. Environ Res, 2009, 109: 22-8. PMID:18945425.

\section{Reasons for Exclusion}

Independent variable

Independent variable

Independent variable

Independent variable, dependent variable

Independent variable

Independent variable 


\section{Excluded Citations}

121 Halldorsson, T. I., Thorsdottir, I., Meltzer, H. M. et al. Linking exposure to polychlorinated biphenyls with fatty fish consumption and reduced fetal growth among Danish pregnant women: a cause for concern?. Am J Epidemiol, 2008, 168: 958-65. PMID:18718897.

\section{2} Hankin, Me, Symonds, Em. Body weight, diet and pre-eclamptic toxaemia of pregnancy. gynaecology, 1962, 4: 156-60

123 Harper, V., Maclnnes, R., Campbell, D. et al. Increased birth weight in northerly islands: is fish consumption a red herring?. Bmj, 1991, 303. PMID:1878642.

\begin{tabular}{|c|c|c|}
\hline & & \\
\hline 124 & $\begin{array}{l}\text { Hatfield, Hm, Dunstan, Ja, Hayes, L et al. Dietary N-3 } \\
\text { polyunsaturated fatty acid (PUFA) supplementation during } \\
\text { pregnancy is associated with changes in cord blood (CB) } \\
\text { progenitor numbers and responsiveness to IL-5 in infants at risk of } \\
\text { atopy [Abstract]. Journal of allergy and clinical immunology, 2003, } \\
111\end{array}$ & Study design \\
\hline 125 & $\begin{array}{l}\text { Haugen, M., Brantsaeter, A. L., Trogstad, L. et al. Vitamin D } \\
\text { supplementation and reduced risk of preeclampsia in nulliparous } \\
\text { women. Epidemiology, 2009, 20: } 720-6 \text {. PMID:19451820. }\end{array}$ & Independent variable \\
\hline 126 & $\begin{array}{l}\text { Hayashi, Tt, Phitaksphraiwan, P, Willson, Jr. Effects of diet and } \\
\text { diuretic agents in pregnancy toxemias. Obstet Gynecol, 1963, 22: } \\
327-34\end{array}$ & Date \\
\hline 127 & $\begin{array}{l}\text { Healthy diet halves the risk of diabetes after pregnancy. Kidney } \\
\text { Care, 2013, 10:6-6 }\end{array}$ & Dependent variable \\
\hline 128 & $\begin{array}{l}\text { Hegsted, D. M. What is a healthful diet?. Prim Care, 1982, 9: 445- } \\
\text { 73. PMID:6924383. }\end{array}$ & Dependent variable \\
\hline 129 & $\begin{array}{l}\text { Heim, T. Energy and lipid requirements of the fetus and the } \\
\text { preterm infant. J Pediatr Gastroenterol Nutr, 1983, . } \\
\text { PMID: } 6417303 .\end{array}$ & Independent variable, study design \\
\hline 130 & $\begin{array}{l}\text { Hellmuth, C., Lindsay, K. L., Uhl, O. et al. Association of maternal } \\
\text { prepregnancy BMI with metabolomic profile across gestation. Int J } \\
\text { Obes (Lond), 2017, 41: 159-169. PMID:27569686. }\end{array}$ & Dependent variable \\
\hline
\end{tabular}

\section{Reasons for Exclusion}

\section{Independent variable}

\section{Date}

Independent variable

(1) 


\section{Excluded Citations}

131 Hennessy, M. D., Volpe, S. L., Sammel, M. D. et al. Skipping meals and less walking among African Americans diagnosed with preterm labor. J Nurs Scholarsh, 2010, 42: 147-55.

PMID:20618599.

132 Heppe, D. H., Steegers, E. A., Timmermans, S. et al. Maternal fish Independent variable consumption, fetal growth and the risks of neonatal complications:

the Generation R Study. Br J Nutr, 2011, 105: 938-49.

PMID:21266095.

133 Hernandez-Diaz, S., Boeke, C. E., Romans, A. T. et al. Triggers of Independent variable, study design spontaneous preterm delivery--why today?. Paediatr Perinat Epidemiol, 2014, 28: 79-87. PMID:24384058.

134 Herrera, Mg, Mora, Jo, Paredes, B et al. Maternal weight/height and the effect of food supplementation during pregnancy and lactation. Maternal Nutrition during Pregnancy and Lactation. A Nestle Foundation Workshop; 1979 April 26-27; Lausanne Switzerland, 1980, : 252-63

135 Hoff, C., Wertelecki, W., Reyes, E. et al. Diet, blood pressure, and Independent variable hematologic variables of nulliparous women attending a prenatal clinic. Obstet Gynecol, 1986, 67: 868-72. PMID:3703412.

136 Hoffman, D, Uauy, R, Birch, D et al. Essentiality of dietary docosahexaenoic acid (dha) for optimal visual maturation in preterm infants: plasma and red blood cell (rbc) fatty acid profiles. lovs, 1992, 33

137 Hoffman, Dr, Uauy, R. Essentiality of dietary $n-3$ fatty acids for premature infants; plasma and red blood cell fatty acid composition. Lipids, 1992, 27: 886-95

138 Hollingsworth, D. R., Ney, D., Stubblefield, N. et al. Metabolic and therapeutic assessment of gestational diabetes by two-hour and twenty-four-hour isocaloric meal tolerance tests. Diabetes, 1985, : 81-7. PMID:3888746.

139 Hook, E. B. Influence of pregnancy on dietary selection. Int J Obes, Study design

1980, 4: 338-40. PMID:7419353.

\section{Reasons for Exclusion}

Independent variable

Independent variable, not peer reviewed

Dependent variable

Independent variable

Dependent variable 


\section{Excluded Citations}

140 Horan, M. K., McGowan, C. A., Gibney, E. R. et al. Maternal low glycaemic index diet, fat intake and postprandial glucose influences neonatal adiposity--secondary analysis from the ROLO study. Nutr J, 2014, 13. PMID:25084967.

141 Horan, M. K., McGowan, C. A., Gibney, E. R. et al. Maternal Nutrition and Glycaemic Index during Pregnancy Impacts on Offspring Adiposity at 6 Months of Age--Analysis from the ROLO Randomised Controlled Trial. Nutrients, 2016, 8. PMID:26742066.

142 Horan, M. K., McGowan, C. A., Gibney, E. R. et al. Maternal nutrition and glycaemic index during pregnancy impacts on offspring adiposity at 6 months of ageâ€"analysis from the ROLO randomised controlled trial. Nutrients, 2016, 8.

143 Huh, S. Y., Rifas-Shiman, S. L., Kleinman, K. P. et al. Maternal protein intake is not associated with infant blood pressure. Int $\mathrm{J}$ Epidemiol, 2005, 34: 378-84. PMID:15576466.

144 Hui, A. L., Ludwig, S. M., Gardiner, P. et al. Community-based exercise and dietary intervention during pregnancy: A pilot study. Canadian Journal of Diabetes, 2006, 30: 169-175 145 Hui, A., Back, L., Ludwig, S. et al. Lifestyle intervention on diet and Independent variable women under a randomized controlled trial. Obstetrical and Gynecological Survey, 2012, 67: 263-264
146 lyengar, L. Effects of dietary supplements late in pregnancy on the Date expectant mother and her newborn. Indian Journal of Medical Research, 1967, 55: 85-9

147 Jedrychowski, W., Perera, F., Mrozek-Budzyn, D. et al. Higher fish Independent variable consumption in pregnancy may confer protection against the harmful effect of prenatal exposure to fine particulate matter. Ann Nutr Metab, 2010, 56: 119-26. PMID:20134157.

\section{Reasons for Exclusion}

Independent variable

Independent variable, dependent variable

Duplicate

Independent variable

Independent variable

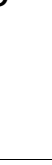




\section{Excluded Citations}

148 Jing, W., Huang, Y., Liu, X. et al. The effect of a personalized intervention on weight gain and physical activity among pregnant women in China. Int J Gynaecol Obstet, 2015, 129: 138-41. PMID:25697965.

149 Johnson, A. A., Knight, E. M., Edwards, C. H. et al. Dietary intakes, anthropometric measurements and pregnancy outcomes. J Nutr, 1994, 124. PMID:8201444.

150 Jovanovic-Peterson, L, Durak, Ep, Peterson, Cm. Randomized trial Health status of diet vs diet plus cardiovascular conditioning on glucose levels in gestational diabetes. Am J Obstet Gynecol, 1989, 161: 415-9

151 Jovanovic-Peterson, L., Peterson, C. M. Turning point in the management of pregnancies complicated by diabetes.

Normoglycemia with self blood glucose monitoring of diet and insulin dosing. ASAIO Trans, 1990, 36: 799-804. PMID:2268482.

152 Jowett, N. I., Nichol, S. G. Diabetic pregnancy. Midwives Chron, 1987, 100: 33-6. PMID:3645266.

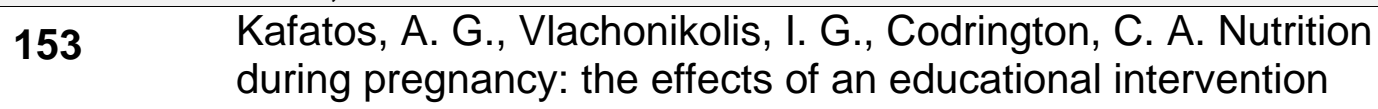
program in Greece. Am J Clin Nutr, 1989, 50: 970-9. PMID:2816804.

154 Kalhan, S. C., Tserng, K. Y., Gilfillan, C. et al. Metabolism of urea and glucose in normal and diabetic pregnancy. Metabolism, 1982, 31: 824-33. PMID:7098852.

155 Kaseb, F., Kimiagar, M., Ghafarpoor, M. et al. Effect of traditional food supplementation during pregnancy on maternal weight gain and birthweight. Int J Vitam Nutr Res, 2002, 72: 389-93. PMID:12596505.

156 Kelleher, C. C., Viljoen, K., Khalil, H. et al. Longitudinal follow-up of the relationship between dietary intake and growth and development in the Lifeways cross-generation cohort study 20012013. Proc Nutr Soc, 2014, 73: 118-31. PMID:24300176.

\section{Reasons for Exclusion}

Independent variable

Independent variable

Independent variable, study design

Study design

Independent variable

Dependent variable

Independent variable

Study design 


\section{Excluded Citations}

157 Kesmodel, U., Olsen, S. F., Salvig, J. D. Marine n-3 fatty acid and calcium intake in relation to pregnancy induced hypertension, intrauterine growth retardation, and preterm delivery. A casecontrol study. Acta Obstet Gynecol Scand, 1997, 76: 38-44. PMID:9033242.

158 Khoury, J, Haugen, G, Tonstad, S et al. Effect of an antiatherogenic diet on maternal and fetal Doppler velocimetry: a randomized clinical trial. 35th Nordic Congress of Obstetrics and Gynecology; 2006 May 23-25; Goteburg, Sweden, 2008,

159 Khoury, J, Henriksen, T, Seljeflot, I et al. Effects of a cholesterollowering diet during pregnancy on cardiovascular risk factors and pregnany outcome: a randomized clinical trial [Study design]. Atherosclerosis. Supplements, 2006, 7

160 Khoury, J., Haugen, G., Tonstad, S. et al. Effect of a cholesterollowering diet during pregnancy on maternal and fetal Doppler velocimetry: the CARRDIP study. Am J Obstet Gynecol, 2007, 196. PMID:17547890.

161 Kinnunen, T. I., Pasanen, M., Aittasalo, M. et al. Preventing excessive weight gain during pregnancy - a controlled trial in primary health care. Eur J Clin Nutr, 2007, 61: 884-91. PMID:17228348.

162 Kinnunen, T. I., Puhkala, J., Raitanen, J. et al. Effects of dietary counselling on food habits and dietary intake of Finnish pregnant women at increased risk for gestational diabetes - a secondary analysis of a cluster-randomized controlled trial. Matern Child Nutr, 2014, 10: 184-97. PMID:22735030.

163 Kizirian, N. V., Kong, Y., Muirhead, R. et al. Effects of a low-

glycemic index diet during pregnancy on offspring growth, body
composition, and vascular health: a pilot randomized controlled

glycemic index diet during pregnancy on offspring growth, body
composition, and vascular health: a pilot randomized controlled trial. Am J Clin Nutr, 2016, 103: 1073-82. PMID:26936333.

\section{Reasons for Exclusion}

Independent variable

\section{Not peer-reviewed}

Study design

Independent variable

Independent variable

Independent variable, dependent variable Independent variable 


\section{Excluded Citations}

164 Klebanoff, M. A., Harper, M., Lai, Y. et al. Fish consumption, erythrocyte fatty acids, and preterm birth. Obstet Gynecol, 2011, 117: 1071-7. PMID:21508745.

165 Knudsen, V. K., Heitmann, B. L., Halldorsson, T. I. et al. Materna dietary glycaemic load during pregnancy and gestational weight gain, birth weight and postpartum weight retention: a study within the Danish National Birth Cohort. Br J Nutr, 2013, 109: 1471-8. PMID:22906835.

166 Knuist, M., Bonsel, G. J., Zondervan, H. A. et al. Low sodium die and pregnancy-induced hypertension: a multi-centre randomised controlled trial. Br J Obstet Gynaecol, 1998, 105: 430-4. PMID:9609271.

167 Koivusalo, S. B., Rono, K., Klemetti, M. M. et al. Gestational Diabetes Mellitus Can Be Prevented by Lifestyle Intervention: The Finnish Gestational Diabetes Prevention Study (RADIEL): A Randomized Controlled Trial. Diabetes Care, 2016, 39: 24-30. PMID:26223239.

168 Kokanali, M. K., Tokmak, A., Kaymak, O. et al. The effect of treatment on pregnancy outcomes in women with one elevated oral glucose tolerance test value. Ginekol Pol, 2014, 85: 748-53. PMID:25546925.

169 Kolu, P., Raitanen, J., Rissanen, P. et al. Cost-effectiveness of lifestyle counselling as primary prevention of gestational diabetes mellitus: findings from a cluster-randomised trial. PLoS One, 2013, 8. PMID:23457562.

170 Korpi-Hyovalti, E., Schwab, U., Laaksonen, D. E. et al. Effect of intensive counselling on the quality of dietary fats in pregnant women at high risk of gestational diabetes mellitus. Br J Nutr, 2012, 108: 910-7. PMID:22093485.

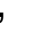

\title{
Reasons for Exclusion
}

Independent variable

Independent variable

\author{
Independent variable
}

Independent variable

(n)

Independent variable

Ind

\section{Independent variable}

Inde

\author{
Independent variable
}

Independent variable

\author{
Independent variable
}




\section{Excluded Citations}

Reasons for Exclusion

171 Kubota, K., Itoh, H., Tasaka, M. et al. Changes of maternal dietary Independent variable intake, bodyweight and fetal growth throughout pregnancy in pregnant Japanese women. J Obstet Gynaecol Res, 2013, 39: 1383-90. PMID:23815608.

172 Kumar, P., Nangia, S., Saili, A. et al. Growth and morbidity Dependent variable patterns of exclusively breast-fed preterm babies. Indian Pediatr, 1999, 36: 296-300. PMID:10713842.

173 Lakin, V., Haggarty, P., Abramovich, D. R. et al. Dietary intake and Independent variable tissue concentration of fatty acids in omnivore, vegetarian and diabetic pregnancy. Prostaglandins Leukot Essent Fatty Acids, 1998, 59: 209-20. PMID:9844995.

174 Langley-Evans, A. J., Langley-Evans, S. C. Relationship between Independent variable maternal nutrient intakes in early and late pregnancy and infants weight and proportions at birth: prospective cohort study. J R Soc Promot Health, 2003, 123: 210-6. PMID:14669495.

\begin{tabular}{lll}
175 & $\begin{array}{l}\text { Laraia, B. A., Siega-Riz, A. M., Kaufman, J. S. et al. Proximity of } \\
\text { supermarkets is positively associated with diet quality index for } \\
\text { pregnancy. Prev Med, 2004, 39: 869-75. PMID:15475018. }\end{array}$ & Dependent variable \\
\hline 176 & $\begin{array}{l}\text { Latva-Pukkila, U., Isolauri, E., Laitinen, K. Dietary and clinical } \\
\text { impacts of nausea and vomiting during pregnancy. J Hum Nutr } \\
\text { Diet, 2010, 23: 69-77. PMID:19943842. }\end{array}$ & Dependent variable \\
& $\begin{array}{l}\text { Leblance, H., Passa, P. Diabetes and pregnancy. Revue du } \\
\text { Praticien - Medecine Generale, 1992, 6: 577-582 }\end{array}$ & Study design, language \\
\hline 177 & Lechtig, A, Habicht, Jp, Delgado, H et al. Effect of food & Date \\
& supplementation during pregnancy on birthweight. Pediatrics, \\
& 1975, 56: 508-20 & \\
\hline 179 & Lenders, C. M., Hediger, M. L., Scholl, T. O. et al. Gestational age & Independent variable \\
& and infant size at birth are associated with dietary sugar intake \\
& among pregnant adolescents. J Nutr, 1997, 127: 1113-7. & \\
& PMID:9187625.
\end{tabular}




\section{Excluded Citations}

180 Ley, S. H., Hanley, A. J., Retnakaran, R. et al. Effect of macronutrient intake during the second trimester on glucose metabolism later in pregnancy. Am J Clin Nutr, 2011, 94: 1232-40.

PMID:21955650.

181 L'Heureux, J. Got sugar? Tips on preventing diabetes. Posit Living, Study design 2002, 11: 12-4. PMID:12083048.

182 Li, S., Zhu, Y., Chavarro, J. E. et al. Healthful Dietary Patterns and Dependent variable the Risk of Hypertension Among Women With a History of

Gestational Diabetes Mellitus: A Prospective Cohort Study.

Hypertension, 2016, 67: 1157-65. PMID:27091899.

183 Lilja, G, Dannaeus, A, Foucard, T et al. Effects of maternal diet during late pregnancy and lactation on the development of atopic disease in infants up to 18 months of age - in-vivo results. Clinical and Experimental Allergy, 1989, 19: 473-9

184 Liu, X., Lv, L., Zhang, H. et al. Folic acid supplementation, dietary folate intake and risk of preterm birth in China. European Journal of Nutrition, 2016, 55: 1411-1422

185 Lorber, D. Gestational diabetes: The hidden epidemic. Female Study design

$186 \quad$ Luoto, $R$, Nermes, M, Laitinen, $\mathrm{K}$ et al. Impact of Maternal Not peer-reviewed

Probiotic-Supplemented Dietary Counselling on Pregnancy

Outcome and Prenatal and Postnatal Growth: A Double-Blind,

Placebo-Controlled Study. Pediatric Academic Societies Annual

Meeting; 2009 May 2 5; Baltimore MD, United States, 2009,

187 MacGillivray, I. Aetiology of pre-eclampsia. Br J Hosp Med, 1981, Independent variable, study design 26. PMID:7296126.

188 MacNeill, S., Dodds, L., Hamilton, D. C. et al. Rates and risk factors for recurrence of gestational diabetes. Diabetes Care, 2001, 24: 659-62. PMID:11315827.

\section{Reasons for Exclusion}

Independent variable, study design

Dependent variable

Independent variable, study design

Independent variable




\section{Excluded Citations}

Reasons for Exclusion $189 \begin{aligned} & \text { Mahony, R, Byrne, J, Curran, S et al. A pilot study of the feasibility Not peer-reviewed } \\ & \text { of a randomised trial of low glycaemic diet versus normal diet from }\end{aligned}$ early pregnancy in euglycaemic women. Arch Dis Child Fetal Neonatal Ed, 2008, 93

190 Makela, J., Lagstrom, H., Kaljonen, A. et al. Hyperglycemia and lower diet quality in pregnant overweight women and increased infant size at birth and at 13 months of age--STEPS study. Early Hum Dev, 2013, 89: 439-44. PMID:23375946.

191 Maresh, M, Alderson, C, Beard, Rw et al. Comparison of insulin Independent variable, not peer reviewed against diet treatment in the management of abnormal carbohydrate tolerance in pregnancy. Nutrition in Pregnancy.

Proceedings of 10th Study Group of the Rcog; 1983, 1983, : 25567

192 Mariscal-Arcas, M., Rivas, A., Monteagudo, C. et al. Proposal of a Dependent variable Mediterranean diet index for pregnant women. Br J Nutr, 2009, 102: 744-9. PMID:19243664.

193 Markovic, T. P., Muirhead, R., Overs, S. et al. Randomized Independent variable Controlled Trial Investigating the Effects of a Low-Glycemic Index Diet on Pregnancy Outcomes in Women at High Risk of Gestational Diabetes Mellitus: The Gl Baby 3 Study. Diabetes Care, 2016, 39: 31-8. PMID:26185283.

194 Marshall, J. Infant feeding: 8. Breastfeeding premature babies. Dependent variable Pract Midwife, 2013, 16. PMID:23789255.

195 Martin, C. L., Siega-Riz, A. M., Sotres-Alvarez, D. et al. Maternal Study design Dietary Patterns are Associated with Lower Levels of Cardiometabolic Markers during Pregnancy. Paediatr Perinat Epidemiol, 2016, 30: 246-55. PMID:26848932.

196 Maten, Gd, Hammen, Rm, Visman, L et al. Effects of a sodium restricted diet during pregnancy on maternal blood pressure and zinc status. J Perinat Med, 1992, 20 


\section{Excluded Citations}

197 Mathews, F., Yudkin, P., Neil, A. Influence of maternal nutrition on outcome of pregnancy: prospective cohort study. Bmj, 1999, 319: 339-43. PMID:10435950.

198 Mathewson, M. Women diagnosed with pregnancy-induced hypertension (pre-eclampsia) should be placed on sodium restricted diets. Crit Care Nurse, 1983, 3. PMID:6552952.

199 McFadyen, A. Intervention in mothers with eating disorders and their babies (controlled trial). National Research Register, 2000,

200 McGowan, C. A., Walsh, J. M., Byrne, J. et al. The influence of a low glycemic index dietary intervention on maternal dietary intake, glycemic index and gestational weight gain during pregnancy: a randomized controlled trial. Nutr J, 2013, 12. PMID:24175958.

201 McGuire, Mk, Burgert, SI, Milner, Ja et al. Selenium status of infants is influenced by supplementation of formula or maternal diets. American Journal of Clinical Nutrition, 1993, 58: 643-8

202 Meinila, J., Koivusalo, S. B., Valkama, A. et al. Nutrient intake of pregnant women at high risk of gestational diabetes. Food Nutr Res, 2015, 59. PMID:25994096.

203 Meinila, J., Valkama, A., Koivusalo, S. B. et al. Healthy Food Intake Index (HFII) - Validity and reproducibility in a gestationaldiabetes-risk population. BMC Public Health, 2016, 16. PMID:27475905.

204 Meltzer, H. M., Brantsaeter, A. L., Nilsen, R. M. et al. Effect of dietary factors in pregnancy on risk of pregnancy complications: results from the Norwegian Mother and Child Cohort Study. Am J Clin Nutr, 2011, 94. PMID:21543541.

205 Mendelson, R., Dollard, D., Hall, P. et al. The impact of the Healthiest Babies Possible Program on maternal diet and pregnancy outcome in underweight and overweight clients. J Can Diet Assoc, 1991, 52: 229-34. PMID:10116012.

\section{Reasons for Exclusion}

Independent variable

Study design

Not peer-reviewed

Independent variable

Independent variable

Dependent variable

Dependent variable

Study design

Independent variable 


\section{Excluded Citations}

\section{Reasons for Exclusion}

206 Mendez, M. A., Plana, E., Guxens, M. et al. Seafood consumption Independent variable in pregnancy and infant size at birth: results from a prospective Spanish cohort. J Epidemiol Community Health, 2010, 64: 216-22. PMID:19710045.

207 Mestman, J. H. Outcome of diabetes screening in pregnancy and Independent variable perinatal morbidity in infants of mothers with mild impairment in glucose tolerance. Diabetes Care, 1980, 3: 447-52. PMID:7389561. 208 Mikkelsen, T. B., Osler, M., Orozova-Bekkevold, I. et al. Independent variable Public Health, 2006, 34: 616-22. PMID:17132595.

209 Mikode, M. S., White, A. A. Dietary assessment of middle-income Independent variable pregnant women during the first, second, and third trimesters. J Am Diet Assoc, 1994, 94: 196-9. PMID:8300999.

210 Misra, A., Ray, S., Patrikar, S. A longitudinal study to determine Country association of various maternal factors with neonatal birth weight at a tertiary care hospital. Med J Armed Forces India, 2015, 71: 2703. PMID:26288495.

211 Mitchell, J., Mackerras, D. The traditional humoral food habits of pregnant Vietnamese-Australian women and their effect on birth weight. Aust J Public Health, 1995, 19: 629-33. PMID:8616205.

212 Mohanty, A. F., Thompson, M. L., Burbacher, T. M. et al. Perinat Epidemiol, 2015, 29: 376-87. PMID:26147526.

213 Moldenhauer, J, Guo, S, Liang, R et al. Dietary intake levels of the antioxidants vitamin $\mathrm{c}$ and vitamin e are adequately achieved with standard prenatal vitamin supplementation in high risk pregnancy groups [abstract]. Am J Obstet Gynecol, 2002, 187

214 Moore, V. M., Davies, M. J., Willson, K. J. et al. Dietary composition of pregnant women is related to size of the baby at composition of pregnant women is related to size
birth. J Nutr, 2004, 134: 1820-6. PMID:15226475.

Independent variable

Independent variable

Not peer-reviewed

Independent variable 


\section{Excluded Citations}

215 Morley, R, Lucas, A. Randomised diet in the neonatal period and growth performance until 7.5-8 y of age in preterm children.

American Journal of Clinical Nutrition, 2000, 71: 822-8

$216 \quad$ Morley, R., Lucas, A. Early diet and outcome in prematurely born. Clinical Nutrition, 1993, 12: 6-11

217 Morrison, Ra, Brien, Pms, Micklewright, A. The effect of dietary Not peer-reviewed supplementation with linoleic acid on the development of pregnancy induced hypertension. 4th World Congress of the International Society for the Study of Hypertension in

Pregnancy;1984 June 18-21; Amsterdam, the Neth, 1984,

218 Morrison, Ra, Brien, Pms. The effect of dietary supplementation with prostaglandin precursors in pregnancy induced hypertension (PIH). 5th International Congress of the International Society for the Study of Hypertension in Pregnancy; 1986 7-10 July, Nottingham,, 1986,

219 Morton, N. E., Gulbrandsen, C. L., Rao, D. C. et al. Determinants Independent variable, health status of blood pressure in Japanese-American Families. Hum Genet, 1980, 53: 261-6. PMID:7358393.

220 Moses, R. G., Casey, S. A., Quinn, E. G. et al. Pregnancy and Glycemic Index Outcomes study: effects of low glycemic index compared with conventional dietary advice on selected pregnancy outcomes. Am J Clin Nutr, 2014, 99: 517-23. PMID:24351875.

221 Moses, R. G., Luebcke, M., Davis, W. S. et al. Effect of a lowglycemic-index diet during pregnancy on obstetric outcomes. Am J Clin Nutr, 2006, 84: 807-12. PMID:17023707.

$222 \quad \begin{aligned} & \text { Moses, R. G., Luebke, M., Petocz, P. et al. Maternal diet and } \\ & \text { infant size } 2 \text { y after the completion of a study of a low-glycemic- }\end{aligned}$ index diet in pregnancy [5]. American Journal of Clinical Nutrition, 2007, 86

223 Moses, Rg, Luebke, M, Petocz, P et al. Maternal diet and infant size 2 y after the completion of a study of a low-glycemic-index diet in pregnancy. American Journal of Clinical Nutrition, 2007, 86

\section{Reasons for Exclusion}

Dependent variable

Independent variable, health status

Not peer-reviewed

Independent variable

Independent variable

Duplicate

Dependent variable 


\section{Excluded Citations}

224 Moss, J. L., Harris, K. M. Impact of maternal and paternal preconception health on birth outcomes using prospective couples' data in Add Health. Arch Gynecol Obstet, 2015, 291: 287-98. PMID:25367598.

225 Mullaney, Laura, Brennan, Aisling, Cawley, Shona et al. Relationship between fasting plasma glucose levels and maternal food group and macronutrient intakes in pregnancy. Dietetics, 2016, 73: 441-447

226 Munson, M., Saatkamp, R., West, C. Late preterm infants: steps to Dependent variable success. Neonatal Netw, 2011, 30: 267-70. PMID:21729860.

227 Musaiger, A. O. Food habits of mothers and children in two regions of Oman. Nutr Health, 1996, 11: 29-48. PMID:8817582.

228 Musselman, J. R., Jurek, A. M., Johnson, K. J. et al. Maternal dietary patterns during early pregnancy and the odds of childhood germ cell tumors: A Children's Oncology Group study. Am J Epidemiol, 2011, 173: 282-91. PMID:21098631.

229 Myhre, R., Brantsaeter, A. L., Myking, S. et al. Intakes of garlic and dried fruits are associated with lower risk of spontaneous preterm delivery. J Nutr, 2013, 143: 1100-8. PMID:23700347.

230 Newman, Ak, Deussen, Ar, Moran, Lj et al. The effect of antenatal Not peer-reviewed dietary and lifestyle advice on maternal psychological health in women who are overweight or obese-findings from the limit randomised trial. Journal of Paediatrics and Child Health [abstracts of the 17th Congress of the Perinatal Society of Australia and New Zealand, , 2013, 49

231 Ney, D., Hollingsworth, D. R., Cousins, L. Decreased insulin requirement and improved control of diabetes in pregnant women given a high-carbohydrate, high-fiber, low-fat diet. Diabetes Care, 1982, 5: 529-33. PMID:6329613.

232 Nicholls, M. G. Reduction of dietary sodium in Western Society. Benefit or risk?. Hypertension, 1984, 6: 795-801. PMID:6394485.

\section{Reasons for Exclusion}

\section{Independent variable}

Independent variable

Independent variable, study design

Dependent variable

Independent variable

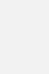




\section{Excluded Citations}

233 Niedhammer, I., Murrin, C., O'Mahony, D. et al. Explanations for social inequalities in preterm delivery in the prospective Lifeways cohort in the Republic of Ireland. Eur J Public Health, 2012, 22: 533-8. PMID:21746747.

234 Odent, M. Land food .. sea food .. brain food. Midwifery Today Childbirth Educ, 1996, : 18-20. PMID:9016057.

235 Olafsdottir, A. S., Skuladottir, G. V., Thorsdottir, I. et al. Maternal diet in early and late pregnancy in relation to weight gain. Int $J$ Obes (Lond), 2006, 30: 492-9. PMID:16331301.

236 Olsen, S. F., Beck, D. N., Kollslid, R. et al. High birth weights in prewar Faroe Islands. J Epidemiol Community Health, 2001, 55. PMID:11160178.

237 Olsen, S. F., Grandjean, P., Weihe, P. et al. Frequency of seafood intake in pregnancy as a determinant of birth weight: evidence for a dose dependent relationship. J Epidemiol Community Health, 1993, 47: 436-40. PMID:8120495.

238 Olsen, S. F., Secher, N. J. Low consumption of seafood in early pregnancy as a risk factor for preterm delivery: prospective cohort study. Bmj, 2002, 324. PMID:11859044.

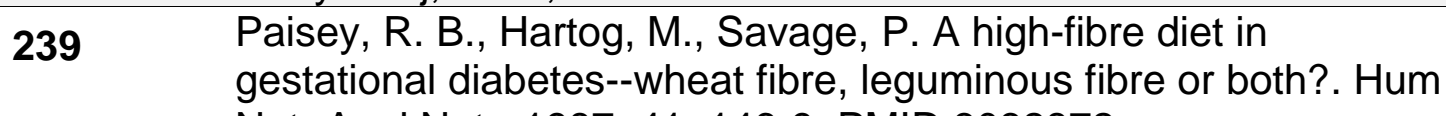
Nutr Appl Nutr, 1987, 41: 146-9. PMID:3032872.

240 Papadopoulou, E., Kogevinas, M., Botsivali, M. et al. Maternal diet, prenatal exposure to dioxin-like compounds and birth outcomes in a European prospective mother-child study (NewGeneris). Sci Total Environ, 2014, 484: 121-8. PMID:24691212. Papazian, T., Hout, H., Sibai, D. et al. Development, reproducib
and validity of a food frequency questionnaire among pregnant
women adherent to the Mediterranean dietary pattern. Clinical Nutrition, 2016, 35: 1550-1556

\section{Reasons for Exclusion}

Independent variable

Study design

Independent variable

Independent variable

Independent variable

Independent variable

Study design

Study design

Dependent variable

$\sqrt{2}$




\section{Excluded Citations}

\section{Reasons for Exclusion}

242 Pedersen, M., von Stedingk, H., Botsivali, M. et al. Birth weight, head circumference, and prenatal exposure to acrylamide from maternal diet: the European prospective mother-child study (NewGeneris). Environ Health Perspect, 2012, 120: 1739-45. PMID:23092936.

243 Pentieva, K., Petrova, S., Ovcharova, D. et al. Influence of some sociodemographic factors and smoking on the risk for intrauterine growth retardation. Khigiena i Zdraveopazvane, 1996, 39: 5-8

244 Perez-Ferre, N., Fernandez, D., Torrejon, M. J. et al. Effect of lifestyle on the risk of gestational diabetes and obstetric outcomes in immigrant Hispanic women living in Spain. J Diabetes, 2012, 4: 432-8. PMID:22742428.

\section{Persson, B, Stangenberg, M, Hansson, $U$ et al. Gestational diabetes mellitus (GDM): comparative evaluation of two treatment} regimens, diet vs insulin and diet. Diabetes, 1985, 34: 101-5

246 Petrella, E., Malavolti, M., Bertarini, V. et al. Gestational weight gain in overweight and obese women enrolled in a healthy lifestyle and eating habits program. J Matern Fetal Neonatal Med, 2014, 27: 1348-52. PMID:24175912.

\begin{tabular}{lll}
\hline 247 & $\begin{array}{l}\text { Petridou, E., Stoikidou, M., Diamantopoulou, M. et al. Diet during } \\
\text { pregnancy in relation to birthweight in healthy singletons. Child } \\
\\
\text { Care Health Dev, 1998, 24: 229-42. PMID:9618037. }\end{array}$ & Independent variable, study design \\
\hline 248 & $\begin{array}{l}\text { Phelan, S., Hart, C., Phipps, M. et al. Maternal behaviors during } \\
\text { pregnancy impact offspring obesity risk. Exp Diabetes Res, 2011, }\end{array}$ & Independent variable \\
& 2011. PMID:22110475. & \\
\hline 249 & $\begin{array}{l}\text { Picaud, Jc, Lapillonne, A, Boucher, P et al. Dietary cholesterol } \\
\text { does not affect vitamin D metabolism in preterm infants : } \\
\text { preliminary results. Pediatr Res, 1999, 45 }\end{array}$ & Dependent variable \\
\end{tabular}

\section{Language}

Health status

Independent variable

Independent variable

Study design 


\section{Excluded Citations}

250 Picone, T. A., Allen, L. H., Olsen, P. N. et al. Pregnancy outcome in North American women. II. Effects of diet, cigarette smoking, stress, and weight gain on placentas, and on neonatal physical and behavioral characteristics. Am J Clin Nutr, 1982, 36: 1214-24. PMID:7148740.

251 Pinto, E., Barros, H., dos Santos Silva, I. Dietary intake and nutritional adequacy prior to conception and during pregnancy: a follow-up study in the north of Portugal. Public Health Nutr, 2009, 12: 922-31. PMID:18752697.

\begin{tabular}{lll}
251 & $\begin{array}{l}\text { nutritional adequacy prior to conception and during pregnancy: a } \\
\text { follow-up study in the north of Portugal. Public Health Nutr, 2009, } \\
\text { 12: 922-31. PMID:18752697. }\end{array}$ & \\
\hline 252 & $\begin{array}{l}\text { Piraquive, J, Grieve, P, Sudha, K et al. Quality of Diet and Central } \\
\text { Nervous System Activity in Low Birth Weight Infants. Pediatric } \\
\text { Academic Societies Annual Meeting, 2013, }\end{array}$ & Independent variable \\
\hline 253 & $\begin{array}{l}\text { Popeski, D., Ebbeling, L. R., Brown, P. B. et al. Blood pressure } \\
\text { during pregnancy in Canadian Inuit: community differences related } \\
\text { to diet. Cmaj, 1991, 145: 445-54. PMID:1878826. }\end{array}$ & Independent variable \\
\hline 254 & $\begin{array}{l}\text { Qiu, C., Coughlin, K. B., Frederick, I. O. et al. Dietary fiber intake } \\
\text { in early pregnancy and risk of subsequent preeclampsia. Am J }\end{array}$ & Independent variable \\
& Hypertens, 2008, 21: 903-9. PMID:18636070. \\
\hline 255 & $\begin{array}{l}\text { Qiu, C., Zhang, C., Gelaye, B. et al. Gestational diabetes mellitus } \\
\text { in relation to maternal dietary heme iron and nonheme iron intake. } \\
\text { Diabetes Care, 2011, 34: 1564-9. PMID:21709295. }\end{array}$ & Independent variable \\
\hline 256 & $\begin{array}{l}\text { Radder, J. K., Terpstra, J. Comparison of postprandial (lunch } \\
\text { tolerance) and postglucose (oral glucose tolerance) blood sugar } \\
\text { values in pregnancy. Eur J Obstet Gynecol Reprod Biol, 1980, 10: }\end{array}$ & Independent variable \\
\hline 257 & $\begin{array}{l}\text { 163-71. PMID:7189481. } \\
\text { Raman, L. Influence of maternal nutritional factors affecting } \\
\text { birthweight. Am J Clin Nutr, 1981, 34: 775-83. PMID:7223693. }\end{array}$ & Country \\
\hline 258 & $\begin{array}{l}\text { Ramon, R., Ballester, F., Aguinagalde, X. et al. Fish consumption } \\
\text { during pregnancy, prenatal mercury exposure, and anthropometric } \\
\text { measures at birth in a prospective mother-infant cohort study in } \\
\text { Spain. Am J Clin Nutr, 2009, 90: 1047-55. PMID:19710189. }\end{array}$ & Independent variable \\
\hline
\end{tabular}

\section{Reasons for Exclusion}

Independent variable

Independent variable, dependent variable 


\section{Excluded Citations}

259 Ramon, R., Ballester, F., Iniguez, C. et al. Vegetable but not fruit intake during pregnancy is associated with newborn anthropometric measures. J Nutr, 2009, 139: 561-7. PMID:19158218.

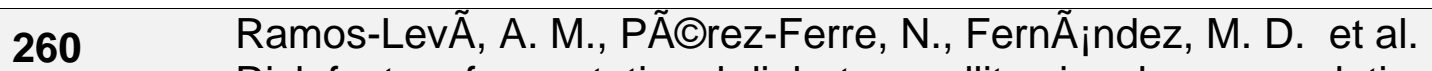
Risk factors for gestational diabetes mellitus in a large population of women living in Spain: Implications for preventative strategies. International Journal of Endocrinology, 2012, 2012

261 Ray, J. G., Mamdani, M. M. Association between folic acid food fortification and hypertension or preeclampsia in pregnancy. Arch Intern Med, 2002, 162: 1776-7. PMID:12153382.

262 Reddy, S., Sanders, T. A., Obeid, O. The influence of maternal vegetarian diet on essential fatty acid status of the newborn. Eur $\mathrm{J}$ Clin Nutr, 1994, 48: 358-68. PMID:8055852.

263 Reece, Ea, Gay, L, DeGennaro, $\mathrm{N}$ et al. A randomized clinical trial of a fiber-enriched diabetic diet vs the standard American Diabetes Association recommended diet in the management of diabetes mellitus in pregnancy. Proceedings of 10th Annual Meeting of Society of Perinatal Obstetricians; 1990 Jan 23-27; Houston, Texas, USA, 1990,

264 Renzaho, A. M., Skouteris, H., Oldroyd, J. Preventing gestational diabetes mellitus among migrant women and reducing obesity and type 2 diabetes in their offspring: a call for culturally competent lifestyle interventions in pregnancy. J Am Diet Assoc, 2010, 110: 1814-7. PMID:21111090.

265 Rhodes, E. T., Pawlak, D. B., Takoudes, T. C. et al. Effects of a low-glycemic load diet in overweight and obese pregnant women: a pilot randomized controlled trial. Am J Clin Nutr, 2010, 92: 1306-15. PMID:20962162.

266 Ribeiro, M. D. Diet and pregnancy toxemia: new thoughts on an old Study design problem. Public Health Rev, 1982, 10: 149-67. PMID:7167640.

Study design

\section{Reasons for Exclusion}

Independent variable

Independent variable, study design

Independent variable

Independent variable

Not peer-reviewed

Independent variable

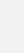




\section{Excluded Citations}

267 Rogers, I., Emmett, P., Baker, D. et al. Financial difficulties, smoking habits, composition of the diet and birthweight in a population of pregnant women in the South West of England. ALSPAC Study Team. Avon Longitudinal Study of Pregnancy and Childhood. Eur J Clin Nutr, 1998, 52: 251-60. PMID:9578337.

268 Ross, Ra, Perlzweig, Wa, Taylor, $\mathrm{Hm}$ et al. A study of certain dietary factors of possible etiologic significance in toxemias of pregnancy. Am J Obstet Gynecol, 1938, 35: 426-40

269 Ruiz-Gracia, T., Duran, A., Fuentes, M. et al. Lifestyle patterns in early pregnancy linked to gestational diabetes mellitus diagnoses when using IADPSG criteria. The St Carlos gestational study. Clin Nutr, 2016, 35: 699-705. PMID:25998584.

270 Rush, D., Stein, Z., Susser, M. Diet in pregnancy: a randomized controlled trial of nutritional supplements. Birth Defects Orig Artic Ser, 1980, 16. PMID:7000197.

271 Saldana, T. M., Siega-Riz, A. M., Adair, L. S. Effect of macronutrient intake on the development of glucose intolerance during pregnancy. Am J Clin Nutr, 2004, 79: 479-86. PMID:14985225.

272 Sanders, T. A., Reddy, S. The influence of a vegetarian diet on the fatty acid composition of human milk and the essential fatty acid status of the infant. J Pediatr, 1992, 120. PMID:1560329.

273 Sauder, K. A., Starling, A. P., Shapiro, A. L. et al. Diet, physical activity and mental health status are associated with dysglycaemia in pregnancy: the Healthy Start Study. Diabet Med, 2016, 33: 6637. PMID:26872289.

274 Saunders, J. B. Investing in healthy babies. NCSL Legisbrief, 2009, Not peer-reviewed

17: 1-2. PMID:19301480.

\section{Reasons for Exclusion}

Independent variable

Date

Independent variable

Independent variable

Independent variable

Dependent variable

Study design 


\section{Excluded Citations}

275 Saunders, L., Guldner, L., Costet, N. et al. Effect of a Mediterranean diet during pregnancy on fetal growth and preterm delivery: results from a French Caribbean Mother-Child Cohort Study (TIMOUN). Paediatr Perinat Epidemiol, 2014, 28: 235-44. PMID:24754337.

276 Savard, N., Levallois, P., Rivest, L. P. et al. Impact of individual and ecological characteristics on small for gestational age births: an observational study in Quebec. Chronic Dis Inj Can, 2014, 34: 46-54. PMID:24618381.

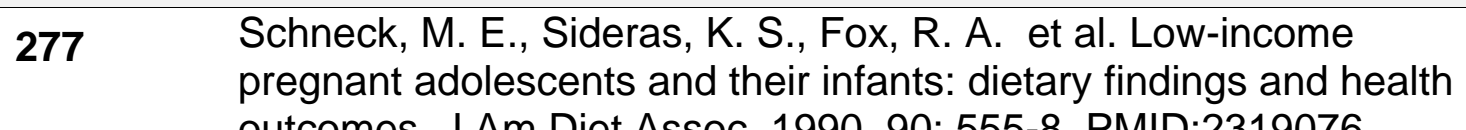
outcomes. J Am Diet Assoc, 1990, 90: 555-8. PMID:2319076.

278 Scott, F. W., Kolb, H. Dietary intervention for diabetes prevention in Study design the neonate. Diabetes Metab Rev, 1998, 14. PMID:9605633.

279 Seely, E. W., Maxwell, C. Cardiology patient page. Chronic hypertension in pregnancy. Circulation, 2007, 115. PMID:17309919.

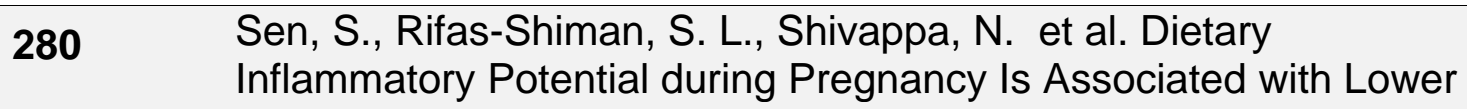
Fetal Growth and Breastfeeding Failure: Results from Project Viva. J Nutr, 2016, 146: 728-36. PMID:26936137.

281 Shin, D., Lee, K. W., Song, W. O. Dietary Patterns during Pregnancy Are Associated with Risk of Gestational Diabetes Mellitus. Nutrients, 2015, 7: 9369-82. PMID:26569302.

282 Siega-Riz, A. M., Herrmann, T. S., Savitz, D. A. et al. Frequency of Independent variable eating during pregnancy and its effect on preterm delivery. Am J Epidemiol, 2001, 153: 647-52. PMID:11282791.

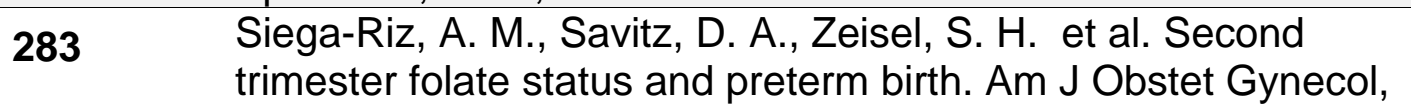
2004, 191: 1851-7. PMID:15592264.

\section{Reasons for Exclusion}

Study design

Independent variable

Independent variable

Independent variable, study design

Independent variable

Study design

Independent variable 


\section{Excluded Citations}

284 Simoes-Wust, A. P., Kummeling, I., Mommers, M. et al. Influence of alternative lifestyles on self-reported body weight and health characteristics in women. Eur J Public Health, 2014, 24: 321-7. PMID:23639916.

285 Sister, MorningStar. Sick pregnancies. Midwifery Today Int Midwife, 2014, : 12-5. PMID:25980101.

286 Smeeth, L., Williams, D. Can a dietary supplement prevent preeclampsia? L-arginine with vitamins show promise, but there are good grounds for caution. Bmj, 2011, 342

287 Smith, L. K., Draper, E. S., Evans, T. A. et al. Associations between late and moderately preterm birth and smoking, alcohol, drug use and diet: a population-based case-cohort study. Arch Dis Child Fetal Neonatal Ed, 2015, 100. PMID:25972442.

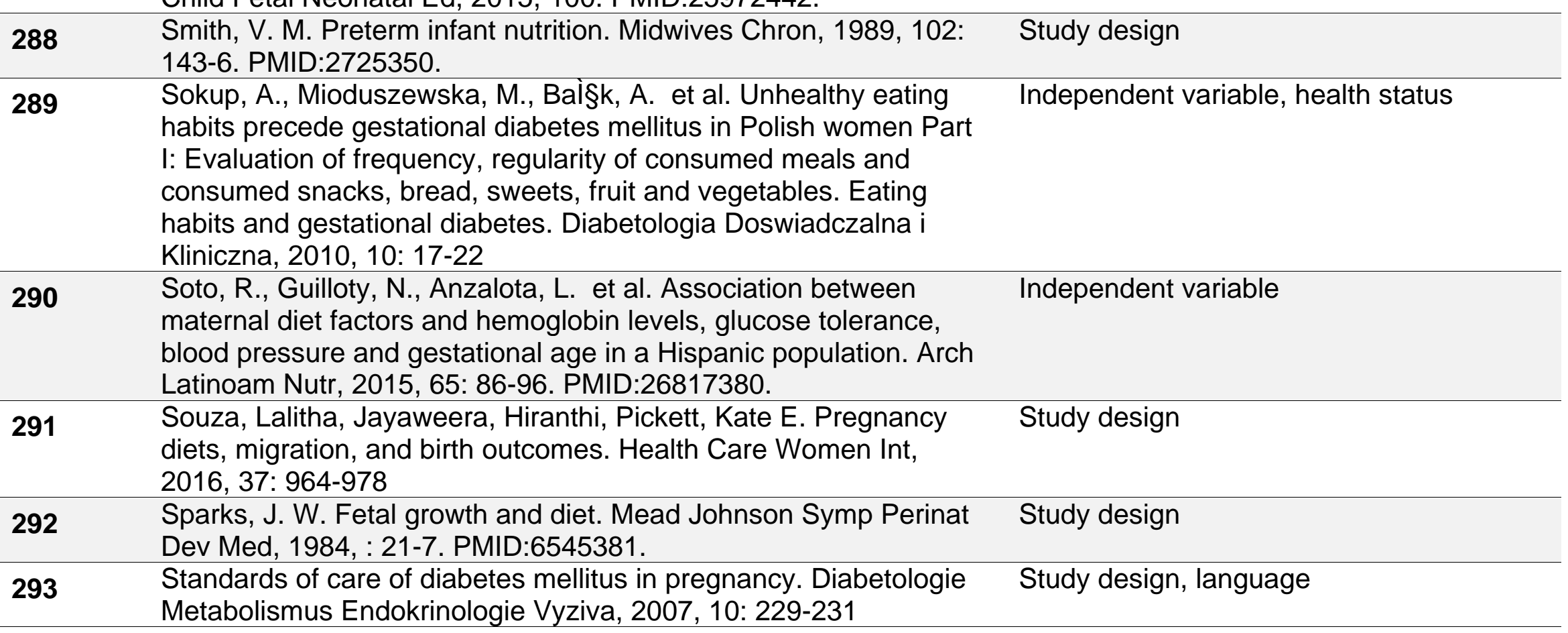

\section{Reasons for Exclusion}

Independent variable

Study design, non peer-reviewed

Independent variable

Study design

Stis

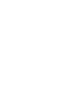




\section{Excluded Citations}

294 Steegers, E. A., Van Lakwijk, H. P., Jongsma, H. W. et al. (Patho)physiological implications of chronic dietary sodium restriction during pregnancy; a longitudinal prospective randomized study. Br J Obstet Gynaecol, 1991, 98: 980-7. PMID:1751444.

295 Steegers, E. A. P., Van Lakwijk, H. P. J. M., Jongsma, H. W. et al. Duplicate (Patho)physiological implications of chronic dietary sodium restriction during pregnancy; a longitudinal prospective randomzied study. Br J Obstet Gynaecol, 1991, 98: 980-987

296 Steegers, Eap, Buul, Eja. Chronic dietary sodium restriction in the prevention of hypertension during pregnancy: preliminary results of a Dutch multicentered trial. 9th International Congress of the International Society for the Study of Hypertension in Pregnancy; 1994 March 15-18; Sydney, A, 1994,

297 Stein, A. Adressing disturbances in the relationship between mothers with eating disorders and their infants: a randomized controlled trial. Personal communication, 2004,

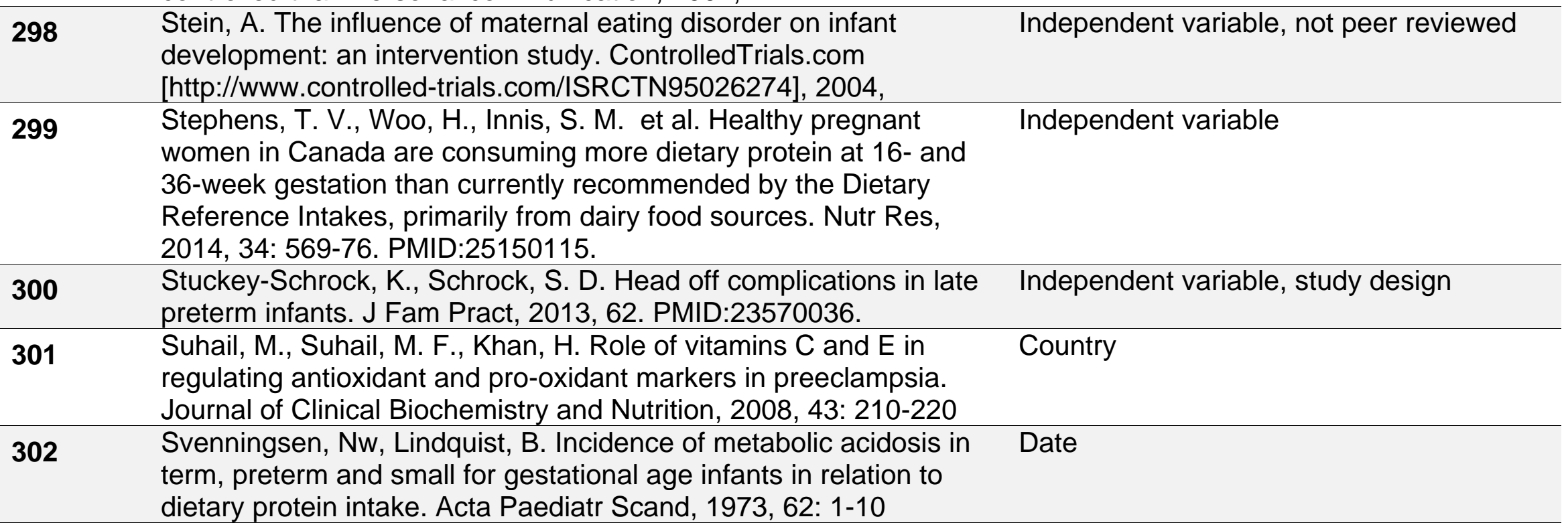

\section{Reasons for Exclusion}

Independent variable

Not peer-reviewed

Independent variable, not peer reviewed

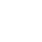




\section{Excluded Citations}

303 Switkowski, K. M., Jacques, P. F., Must, A. et al. Maternal protein intake during pregnancy and linear growth in the offspring. Am J

Clin Nutr, 2016, 104: 1128-1136. PMID:27581477.

304 Symonds, M. E., Budge, H., Edwards, L. J. et al. Maternal nutrition, cortisol and programming of fetal development.

Perinatology, 2002, 4: 67-74

305 Tande, D. L., Ralph, J. L., Johnson, L. K. et al. First trimester dietary intake, biochemical measures, and subsequent gestational hypertension among nulliparous women. J Midwifery Womens Health, 2013, 58: 423-30. PMID:23895215.

\begin{tabular}{|c|c|c|}
\hline 306 & $\begin{array}{l}\text { Tanha, F. D., Mohseni, M., Ghajarzadeh, M. et al. The effects of } \\
\text { healthy diet in pregnancy. Journal of Family and Reproductive } \\
\text { Health, 2013, 7: 121-125 }\end{array}$ & Independent variable, dependent variable \\
\hline 307 & $\begin{array}{l}\text { Taylor, C. M., Golding, J., Emond, A. M. Blood mercury levels and } \\
\text { fish consumption in pregnancy: Risks and benefits for birth } \\
\text { outcomes in a prospective observational birth cohort. Int J Hyg } \\
\text { Environ Health, 2016, 219: 513-20. PMID:27252152. }\end{array}$ & Independent variable \\
\hline 308 & $\begin{array}{l}\text { Thacker, S. M., Petkewicz, K. A. Gestational diabetes mellitus. } \\
\text { U.S. Pharm., 2009, 34: } 43-48\end{array}$ & Study design \\
\hline 309 & $\begin{array}{l}\text { Thomas, B., Ghebremeskel, K., Lowy, C. et al. Nutrient intake of } \\
\text { women with and without gestational diabetes with a specific focus } \\
\text { on fatty acids. Nutrition, 2006, 22: 230-6. PMID:16500549. }\end{array}$ & Independent variable, study design \\
\hline 310 & $\begin{array}{l}\text { Thomas, D. M., Clapp, J. F., Shernce, S. A foetal energy balance } \\
\text { equation based on maternal exercise and diet. J R Soc Interface, } \\
\text { 2008, 5: 449-55. PMID:17895222. }\end{array}$ & Independent variable \\
\hline 311 & $\begin{array}{l}\text { Thompson, J. M., Wall, C., Becroft, D. M. et al. Maternal dietary } \\
\text { patterns in pregnancy and the association with small-for- } \\
\text { gestational-age infants. Br J Nutr, 2010, 103: 1665-73. } \\
\text { PMID:20211035. }\end{array}$ & Study design \\
\hline 312 & $\begin{array}{l}\text { Tielemans, M. J., Erler, N. S., Leermakers, E. T. M. et al. A Priori } \\
\text { and a Posteriori dietary patterns during pregnancy and gestational } \\
\text { weight gain: The generation R study. Nutrients, 2015, 7: 9383-9399 }\end{array}$ & Dependent variable \\
\hline
\end{tabular}

\section{Reasons for Exclusion}

Independent variable

No full text

Independent variable

\author{
(1)
}




\section{Excluded Citations}

313 Tobias, D. K., Zhang, C., Chavarro, J. et al. Healthful dietary patterns and long-term weight change among women with a history of gestational diabetes mellitus. Int J Obes (Lond), 2016, 40: 17481753. PMID:27569683.

314 Tovar, A., Must, A., Bermudez, O. I. et al. The impact of gestational weight gain and diet on abnormal glucose tolerance during pregnancy in Hispanic women. Matern Child Health J, 2009, 13: 520-30. PMID:18597166.

315 Uusitalo, U., Arkkola, T., Ovaskainen, M. L. et al. Unhealthy dietary patterns are associated with weight gain during pregnana
among Finnish women. Public Health Nutr, 2009, 12: 2392-9. PMID:19323867.

316 Valentini, R., Dalfra, M. G., Masin, M. et al. A pilot study on dietary Health status approaches in multiethnicity: two methods compared. Int $\mathrm{J}$ Endocrinol, 2012, 2012. PMID:22505892.

317 Van Buul, B. J. A., Steegers, E. A. P., Van Der Maten, G. D. et al. Independent variable Dietary sodium restriction does not prevent gestational hypertension: A Dutch two-center randomized trial. Hypertension in Pregnancy, 1997, 16: 335-346

\begin{tabular}{lll}
\hline 318 & $\begin{array}{l}\text { van Buul, B. J., Steegers, E. A., Jongsma, H. W. et al. Dietary } \\
\text { sodium restriction in the prophylaxis of hypertensive disorders of } \\
\text { pregnancy: effects on the intake of other nutrients. Am J Clin Nutr, } \\
\text { 1995, 62: 49-57. PMID:7598066. }\end{array}$ & Independent variable \\
\hline 319 & $\begin{array}{l}\text { van der Maten, G. D. Low sodium diet in pregnancy: effects on } \\
\text { maternal nutritional status. Eur J Obstet Gynecol Reprod Biol, } \\
\text { 1995, 61: 63-4. PMID:8549849. }\end{array}$ & Independent variable \\
\hline $\mathbf{3 2 0}$ & $\begin{array}{l}\text { van der Maten, G. D., van Raaij, J. M., Visman, L. et al. Low- } \\
\text { sodium diet in pregnancy: effects on blood pressure and maternal } \\
\text { nutritional status. Br J Nutr, 1997, 77: 703-20. PMID:9175991. }\end{array}$ & Independent variable \\
\hline
\end{tabular}

Dependent variable

\section{Reasons for Exclusion}

Dependent variable

Independent variable

Independent variable

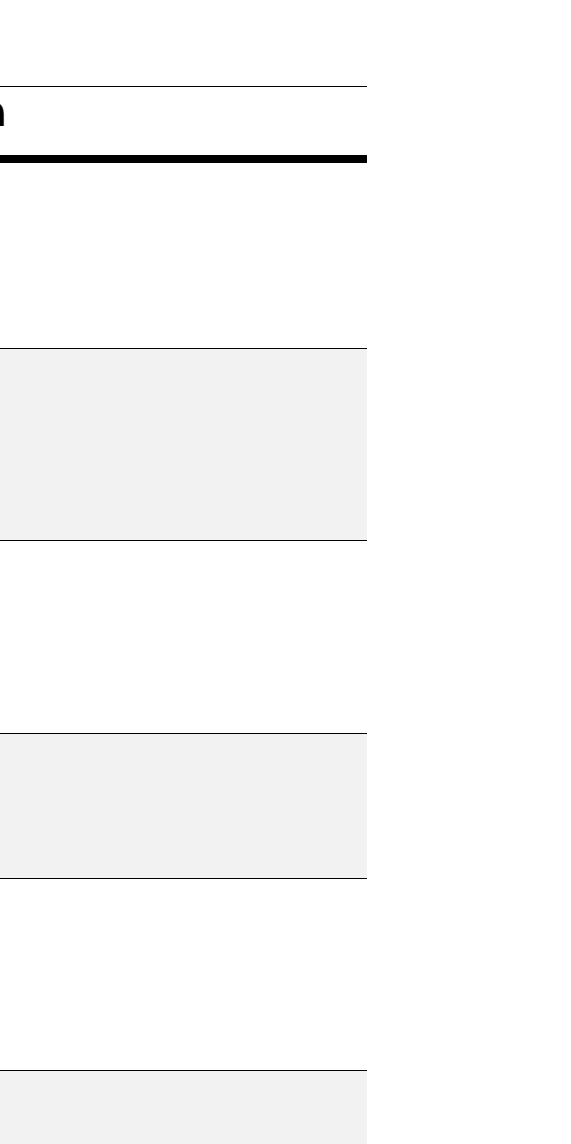




\section{Excluded Citations}

321 Vejrup, K., Brantsaeter, A. L., Knutsen, H. K. et al. Prenatal mercury exposure and infant birth weight in the Norwegian Mother and Child Cohort Study. Public Health Nutr, 2014, 17: 2071-80. PMID:24103413.

322 Vilela, A. A., Pinto Tde, J., Rebelo, F. et al. Association of Prepregnancy Dietary Patterns and Anxiety Symptoms from Midpregnancy to Early Postpartum in a Prospective Cohort of Brazilian Women. J Acad Nutr Diet, 2015, 115: 1626-35. PMID:25769749.

323 Vitolo, Mr, Fraga, Bueno Ms, Mendes, Gama C. Impact of a dietary Language counseling program on the gain weight speed of pregnant women attended in a primary care service. Revista Brasileira de Ginecologia e Obstetricia, 2011, 33: 13-19

324 Wakimoto, Patricia, Akabike, Andrea, King, Janet C. Maternal Nutrition and Pregnancy Outcomeâ€"A Look Back. Nutrition Today, 2015, 50: 221-229

325 Walsh, J. M., Mahony, R. M., Culliton, M. et al. Impact of a low glycemic index diet in pregnancy on markers of maternal and fetal metabolism and inflammation. Reprod Sci, 2014, 21: 1378-81. PMID:24642719.

326 Walsh, J. M., McGowan, C. A., Mahony, R. et al. Low glycaemic index diet in pregnancy to prevent macrosomia (ROLO study): randomised control trial. Bmj, 2012, 345. PMID:22936795.

327 Wang, C., Zhu, W., Wei, Y. et al. Exercise intervention during pregnancy can be used to manage weight gain and improve pregnancy outcomes in women with gestational diabetes mellitus. BMC Pregnancy Childbirth, 2015, 15. PMID:26459271.

328

\section{Reasons for Exclusion}

Independent variable

Dependent variable

Study design, non peer-reviewed

Dependent variable

Independent variable

Independent variable

Weed, S. S. Preeclampsia. Midwifery Today Int Midwife, 2014, : Study design 22-3. PMID:25980104. 


\section{Excluded Citations}

329 Wen, L. M., Simpson, J. M., Rissel, C. et al. Maternal junk food diet during pregnancy as a predictor of high birthweight: findings from the healthy beginnings trial. Birth, 2013, 40: 46-51.

PMID:24635424.

330 Wheeler, S. J., Poston, L., Thomas, J. E. et al. Maternal plasma fatty acid composition and pregnancy outcome in adolescents. $\mathrm{Br} \mathrm{J}$ Nutr, 2011, 105: 601-10. PMID:21269546.

$331 \quad$ Widga, A. C., Lewis, N. M. Defined, in-home, prenatal nutrition PMID:10491673.

332 Williams, C., Highley, W., Ma, E. H. et al. Protein, amino acid, and Independent variable caloric intakes of selected pregnant women. J Am Diet Assoc, 1981, 78: 28-35. PMID:7217557.

$333 \quad \begin{array}{ll}\text { Williams, E. J. Gestational diabetes mellitus and diet control. } & \text { Study design } \\ \text { Diabetes Educ, 1986, 12: 16-7. PMID:3633805. } & \end{array}$

334 Wolff, C. B., Wolff, H. K. Maternal eating patterns and birth weight Study design of Mexican American infants. Nutr Health, 1995, 10: 121-34. PMID:7491165.

335 Wood,. Diet for diabetics: fact vs. hypothesis. Compr Ther, 1980, 6: Study design 56-61. PMID:7408433.

336 Wynn, A. H., Crawford, M. A., Doyle, W. et al. Nutrition of women Independent variable in anticipation of pregnancy. Nutr Health, 1991, 7: 69-88. PMID:2038457.

337 Zielinsky, P., Piccoli,, Vian, I. et al. Maternal restriction of polyphenols and fetal ductal dynamics in normal pregnancy: an open clinical trial. Arq Bras Cardiol, 2013, 101: 217-25. PMID:23949325.

\section{Reasons for Exclusion}

Independent variable

Independent variable

Independent variable

Independent variable 